\title{
Conceptual Design of a Simplified Skid- Mounted Caustic-Side Solvent Extraction Process for Removal of Cesium from Savannah River Site High-Level Waste
}

\section{May 2004}

Prepared by

Joseph F. Birdwell, Jr., R. M. Counce, and Charles O. Slater Oak Ridge National Laboratory

R. A. Pierce and D. D. Walker Savannah River Technology Center 



\section{DOCUMENT AVAILABILITY}

Reports produced after January 1, 1996 are generally available free via the U.S. Department of Energy (DOE) Information Bridge.

Web site http://www.osti.gov/bridge

Reports produced before January 1, 1996 may be purchased by members of the public from the following source.

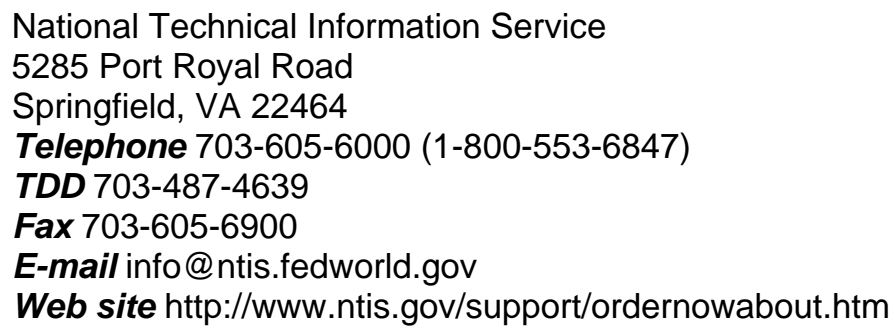

Reports are available to DOE employees, DOE contractors, Energy Technology Data Exchange (ETDE) representatives, and International Nuclear Information System (INIS) representatives from the following source.

Office of Scientific and Technical Information

P.O. Box 62

Oak Ridge, TN 37831

Telephone 865-576-8401

Fax 865-576-5728

E-mail reports@adonis.osti.gov

Web site http://www.osti.gov/contact.html

This report was prepared as an account of work sponsored by an agency of the United States Government. Neither the United States Government nor any agency thereof, nor any of their employees, makes any warranty, express or implied, or assumes any legal liability or responsibility for the accuracy, completeness, or usefulness of any information, apparatus, product, or process disclosed, or represents that its use would not infringe privately owned rights. Reference herein to any specific commercial product, process, or service by trade name trademark, manufacturer, or endorsement recommendation, or favoring by the United States Government or any agency thereof. The views and opinions of authors expressed herein do not necessarily state or reflect those of the United States Government of any agency thereof. 
ORNL/TM-2004/59

\title{
Conceptual Design of a Simplified Skid-Mounted Caustic-Side Solvent Extraction Process for Removal of Cesium from Savannah River Site High-Level Waste
}

\author{
J. F. Birdwell, Jr. \\ R. M. Counce \\ C. O. Slater \\ Oak Ridge National Laboratory \\ R. A. Pierce \\ D. D. Walker \\ Savannah River Technology Center
}

Date Published: May 2004

\author{
Prepared for the \\ DOE Office of Environmental Management \\ DOE Office of Science and Technology \\ Prepared by \\ OAK RIDGE NATIONAL LABORATORY \\ Oak Ridge, Tennessee 37831-6283 \\ managed by \\ UT-BATTELLE, LLC \\ for the \\ U.S. DEPARTMENT OF ENERGY \\ under contract DE-AC05-00OR22725
}





\section{CONTENTS}

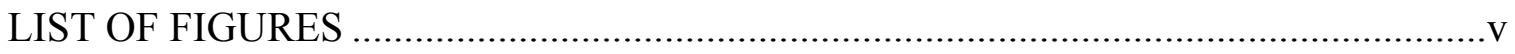

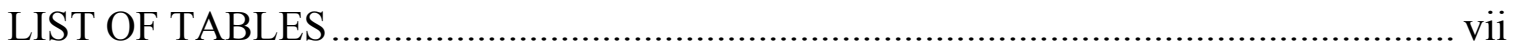

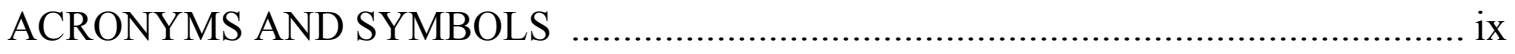

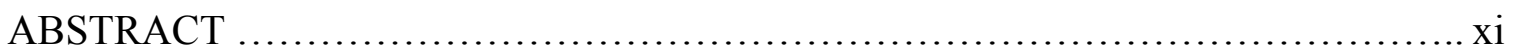

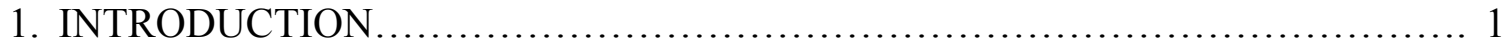

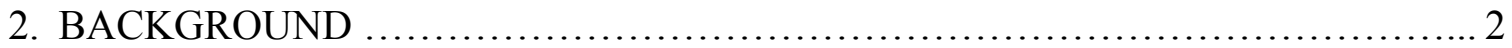

2.1 HLW OVERVIEW ........................................................ 2

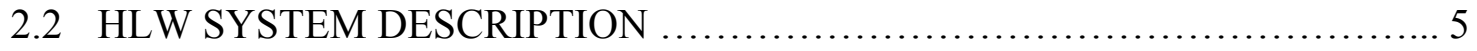

2.3 CAUSTIC-SIDE SOLVENT EXTRACTION PROCESS .....................6

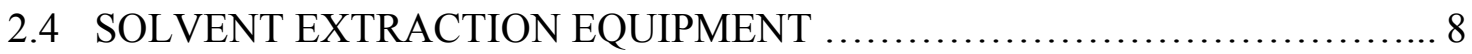

3. PROCESS DESIGN ......................................................... 10

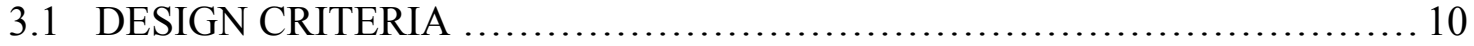

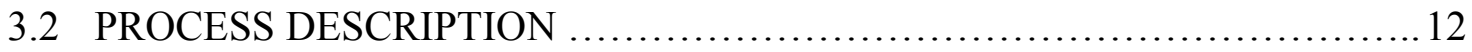

3.3 EQUIPMENT DESCRIPTIONS ........................................ 28

3.3.1 Centrifugal Contactors ................................................ 28

3.3.2 Cross-flow Filters ....................................................... 28

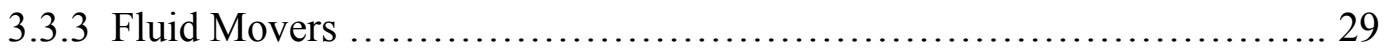

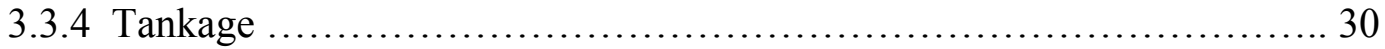

3.4 SYSTEM DEPLOYMENT .............................................. 30

3.4.1 Building 241-96H .............................................. 31

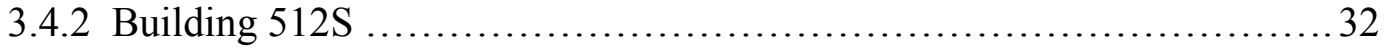

3.4.3 Skid-Mounted Containerized System …............................ 33

3.4.4 Selection of Deployment Concept................................... 34

3.5 SYSTEM MAINTENANCE CONCEPT …............................. 34

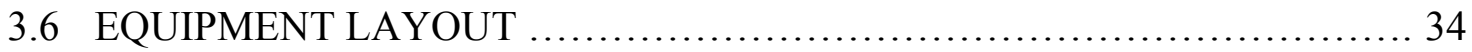

3.7 IMPACTS ON HLW SYSTEM ............................................. 35

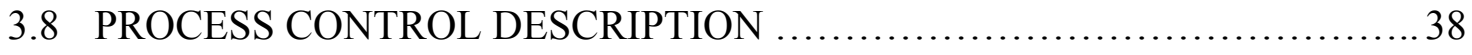

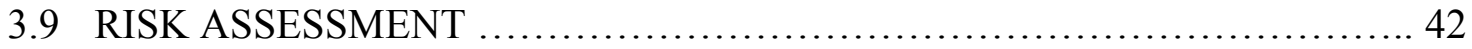


3.9.1 Handling of Hazardous Materials ................................ 43

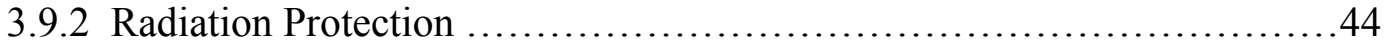

3.9 .3 Criticality ................................................... 45

3.9.4 Release of Gaseous Emissions ..................................45

3.9.5 Accidental Release of Process Solutions ............................. 45

3.9.6 Electrical Equipment ......................................... 46

3.9.7 Use of Rotating Machinery ..................................... 46

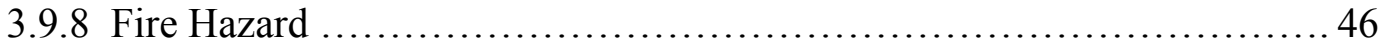

3.9.9 Operational Risks .........................................47

3.10 TECHNICAL UNCERTAINTIES ................................... 47

3.10.1 Processing of Concentrated Feed .................................48

3.10.2 Need for Solvent Loss Mitigation .............................. 48

3.10.3 Contactor Hydraulic and Mass Transfer Performance ................. 49

3.10.4 In-line Gamma Monitoring .................................... 49

4. ECONOMIC ANALYSIS ................................................. 50

4.1 BASIS FOR COMPARISON WITH BASELINE ......................... 50

4.2 FIXED CAPITAL INVESTMENT ESTIMATE ............................ 52

4.3 ESTIMATION OF ANNUAL OPERATING COSTS ........................ 54

4.4 LIFE-CYCLE COSTS AND COST/BENEFIT ANALYSIS ................... 54

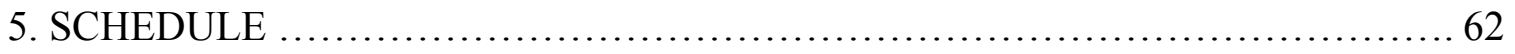

6. DISCUSSION AND CONCLUSIONS ........................................ 62

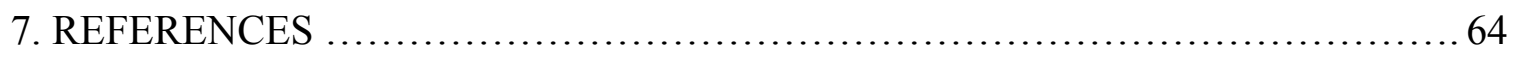

APPENDIX A: DOSE RATE CALCULATIONS ............................. 66

APPENDIX B: PROJECT SCHEDULE .................................... 84 


\section{LIST OF FIGURES}

Figure Page

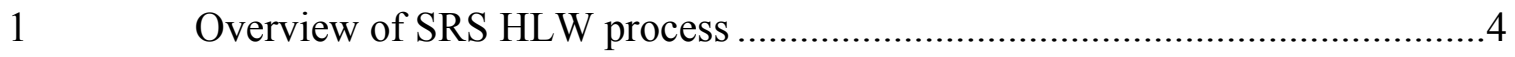

2 Centrifugal contactor cross section with flow patterns ................................

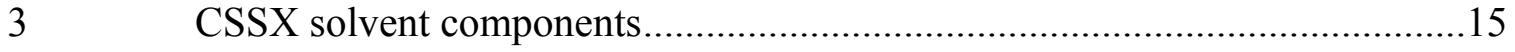

4 Schematic of CSSX-A solvent extraction cascade .....................................16

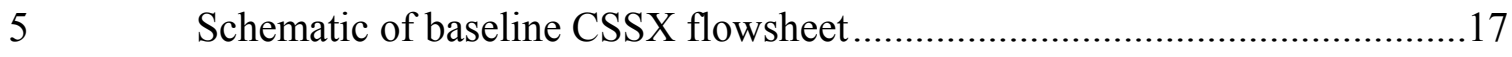

$6 \quad$ Schematic of material balance flowsheet..................................................... 18

7 Mainline process equipment arrangement, based on original system

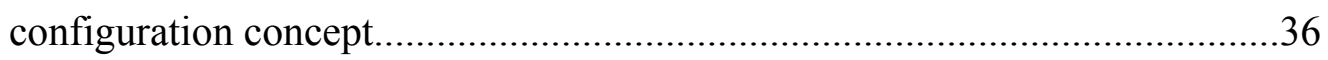

$8 \quad$ Cold chemical makeup area equipment arrangement .................................37

9 Mainline process equipment arrangement, based on alternative

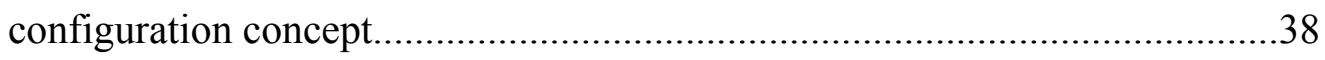

10 Arrangement of aqueous feed and receiving tanks in alternative

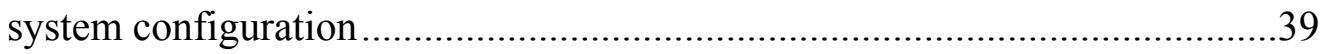

11 CSSX-A system process instrumentation diagram .................................. 41 



\section{LIST OF TABLES}

\section{Table}

Page

CSSX solvent composition

CSSX-A system stream descriptions .

Material balance results, flows in kilogram moles per hour. .20

Material balance stream characterizations in kilogram moles per

cubic meter. .24

Summary of transfers to the SRS HLW system from CSSX-A

Purchased costs of major equipment items ..............................................53

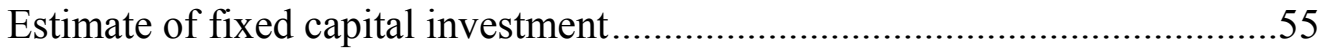

Annual direct operational cost estimate. .56

11 Life-cycle cost as a function of project lifetime .....................................58

12 Cost/benefit analysis for an SWPF CSSX baseline of 74 million gallons.......59

13 Cost/benefit analysis for an SWPF CSSX baseline of 37 million gallons.......60

14 Cost savings based solely on accelerated tank farm closure 



\section{ACRONYMS AND SYMBOLS}

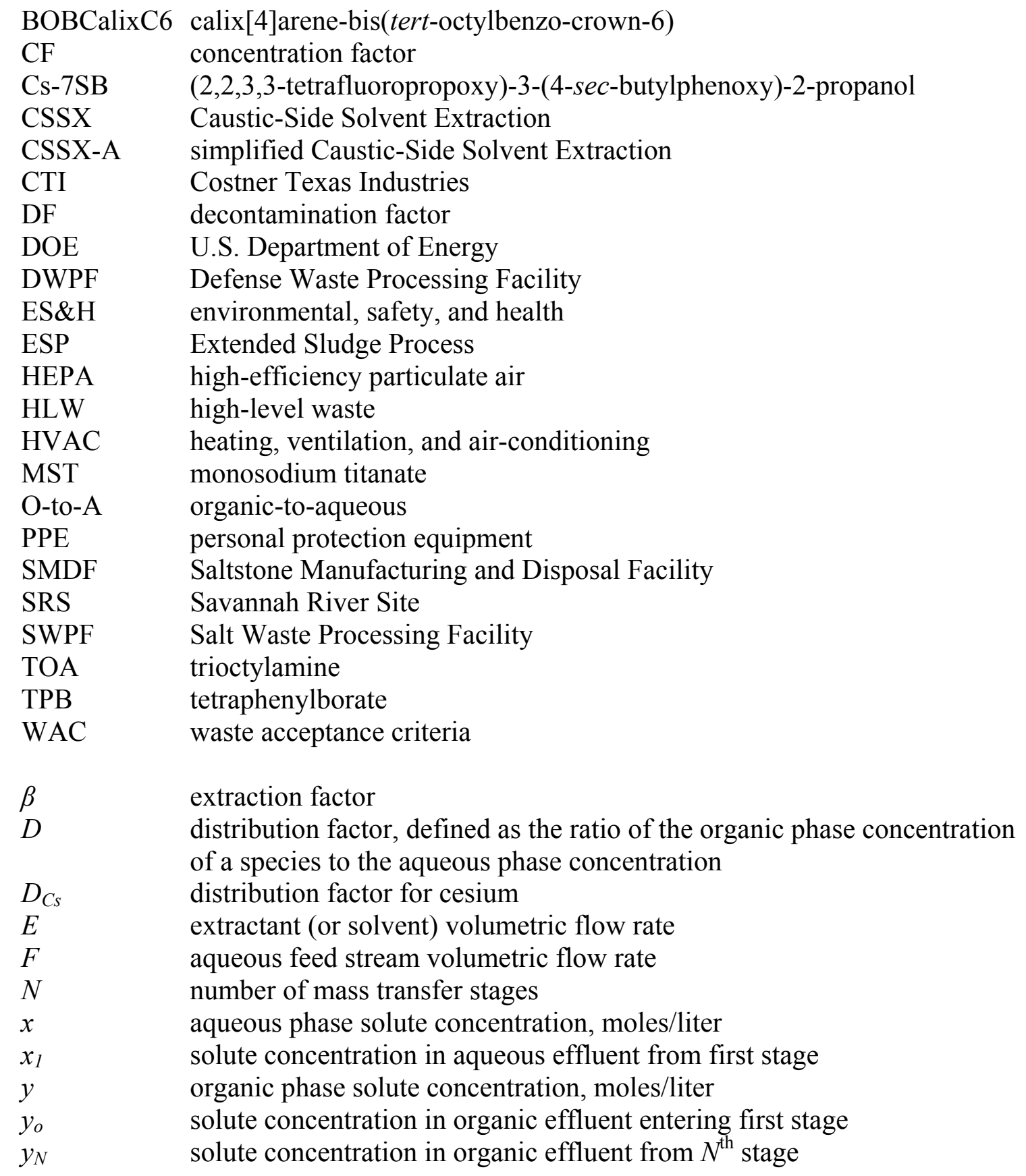





\begin{abstract}
This report presents the results of a conceptual design of a solvent extraction process for the selective removal of ${ }^{137} \mathrm{Cs}$ from high-level radioactive waste currently stored in underground tanks at the U.S. Department of Energy's Savannah River Site (SRS). This study establishes the need for and feasibility of deploying a simplified version of the Caustic-Side Solvent Extraction (CSSX) process; cost/benefit ratios ranging from 33 to 55 strongly support the considered deployment.

Based on projected compositions, 18 million gallons of dissolved salt cake waste has been identified as having ${ }^{137} \mathrm{Cs}$ concentrations that are substantially lower than the worst-case design basis for the CSSX system that is to be deployed as part of the Salt Waste Processing Facility (SWPF) but that does not meet the waste acceptance criteria for immobilization as grout in the Saltstone Manufacturing and Disposal Facility at SRS. Absent deployment of an alternative cesium removal process, this material will require treatment in the SWPF CSSX system, even though the cesium decontamination factor required is far less than that provided by that system.

A conceptual design of a CSSX processing system designed for rapid deployment and having reduced cesium decontamination factor capability has been performed. The proposed accelerated-deployment CSSX system (CSSX-A) has been designed to have a processing rate of 3 million gallons per year, assuming $90 \%$ availability. At a more conservative availability of $75 \%$ (reflecting the novelty of the process), the annual processing capacity is 2.5 million gallons. The primary component of the process is a 20 stage cascade of centrifugal solvent extraction contactors. The decontamination and concentration factors are 40 and 15, respectively. The solvent, scrub, strip, and wash solutions are to have the same compositions as those planned for the SWPF CSSX system. As in the SWPF CSSX system, the solvent and scrub flow rates are equal. The system is designed to facilitate remote operation and direct maintenance.

Two general deployment concepts were considered: (1) deployment in an existing but unused SRS facility and (2) deployment in transportable containers. Deployment in three transportable containers was selected as the preferred option, based on concerns regarding facility availability (due to competition from other processing alternatives) and decontamination and renovation costs.

A risk assessment identified environmental, safety, and health issues that exist. These concerns have been addressed in the conceptual design by inclusion of mitigating system features. Due to the highly developed state of CSSX technology, only a few technical issues remain unresolved; however, none of these issues have the potential to make the technology unviable. Recommended development tasks that need to be performed to address technical uncertainties are discussed in this report.

Deployment of the proposed CSSX-A system provides significant qualitative and quantitative benefits. The qualitative benefits include (1) verification of full-scale contactor performance under CSSX conditions that will support SWPF CSSX design and deployment; (2) development of design, fabrication, and installation experience bases that will be at least partially applicable to the SWPF CSSX system; and (3) availability of the CSSX-A system as a means of providing contactor-based solvent extraction system operating experience to SWPF CSSX operating personnel.
\end{abstract}


Estimates of fixed capital investment, development costs, and annual operating cost for SRS deployment of the CSSX-A system (in mid-2003 dollars) are $\$ 9,165,199$, $\$ 2,734,801$, and $\$ 2,108,820$, respectively. When the economics of the CSSX-A system are compared with those of the baseline SWPF CSSX system, benefit-to-cost ratios ranging from 20 to 47 are obtained. The benefits in the cost/benefit comparison arise from expedited tank closure and reduced engineering, construction, and operating costs for the SWPF CSSX system.

No significant impediments to deployment were determined in the reported analysis, and where technical uncertainties were identified, development tasks to mitigate them are indicated. It is recommended that deployment of the CSSX-A system be pursued in a timely manner in order to derive the greatest possible cost and accelerated treatment benefits. 


\section{INTRODUCTION}

This report presents the results of a conceptual design of a solvent extraction process for the selective removal of cesium from high-level radioactive waste currently stored in underground tanks at the U.S. Department of Energy (DOE) Savannah River Site (SRS). A principal objective of the work was to determine the need for and feasibility of deploying a simplified version of the Caustic-Side Solvent Extraction (CSSX) process that was developed for use in the Salt Waste Processing Facility (SWPF). The proposed system will accept waste that does not meet the ${ }^{137} \mathrm{Cs}$ waste acceptance criteria (WAC) for immobilization as grout in the Saltstone facility at SRS but that does not contain the high cesium concentrations that served as the design basis for the SWPF CSSX system. The second major objective of the subject task was to perform a cost/benefit analysis of the proposed deployment relative to the processing of all stored cesium-containing waste supernates and salt cakes in the SWPF CSSX system.

The SWPF CSSX process was designed to treat high-level waste (HLW) containing cesium over a wide range of concentrations. In addition to feed stream characterizations, performance criteria of the SWPF CSSX system were determined based on very stringent WAC for the Saltstone facility. To comply with the Saltstone WAC on a highest-concentration-cesium waste basis, a system decontamination factor of 40,000 was established as a performance criterion for the SWPF CSSX system. In the baseline SRS HLW treatment concept, all nonsludge waste components are to be treated in the SWPF; actinides and strontium are removed by sorption onto monosodium titanate (MST) and cesium is removed from the filtrate by selective extraction using the CSSX process. The total volume of waste to be treated in this manner would be 85-90 million gallons (based on dissolution to $5.6 \mathrm{MNa}$ ), consisting of supernate and dissolved salt cake. Insoluble sludge would be retrieved from the tanks, washed, and transferred directly to the Defense Waste Processing Facility (DWPF) for vitrification.

To reduce the life-cycle cost of the SWPF and to accelerate dispositioning of radionuclide wastes, SRS developed an alternative remediation approach in which waste is treated to various extents, according to composition. Under this approach, only wastes requiring higher degrees of actinide, strontium, and cesium removal are directed to 
SWPF. Waste supernates and interstitial liquid removed from the undissolved salt cake are expected to comprise the bulk of material requiring treatment in SWPF. After removal of the liquid fraction, the remaining salt cake will be dissolved using either inhibited water or low-activity wastewater, possibly recycle water from DWPF. Dissolved salt cake will be characterized and dispositioned accordingly. Of the total combined volume of supernate, interstitial liquid, and dissolved salt cake, it is anticipated that approximately one-third will require treatment in SWPF; one-third can be dispositioned as low curie salt that requires no treatment prior to immobilization as saltstone; and one-third will require either actinide removal or a degree of cesium removal less than that provided in SWPF, or both. To accelerate processing of this waste, alternative processing pathways to SWPF are being developed. The subject of this report is a proposed alternative for treating waste that requires cesium removal to meet Saltstone WAC but that does not require the level of treatment provided in the SWPF CSSX process.

As part of the overall design effort, safety, health, and environmental aspects of the proposed process have been considered and mitigation technologies have been included in the design where necessary. Technical uncertainties that need to be addressed prior to deployment of the technology have also been identified, the risk each uncertainty poses to successful deployment has been assessed, and approaches for resolving the uncertainties have been proposed.

\section{BACKGROUND}

\subsection{HLW OVERVIEW}

Since the start of operations in 1952, SRS has collected and stored over 100 million gallons of waste in the F-Area and H-Area Tank Farms, commonly known as the HLW system. Incrementally, waste has been neutralized; insoluble sludge has settled; and supernates have been evaporated, resulting in the formation of salt cake. Thus, the current HLW inventory has been reduced to 37 million gallons. Of the total HLW inventory, 3 million gallons is present in the form of insoluble sludge; the remaining 34 million gallons is present as salt cake and supernate. The two tank farms 
contain 51 tanks, two of which have been emptied of HLW and filled with grout for the purpose of stabilization.

The radionuclide inventory of the HLW tank farms is approximately 426 million curies. By component, sludge (3 million gallons) has a radionuclide content of approximately 226 million curies and salt (34 million gallons) has a radionuclide content of approximately 200 million curies. Approximately 197.5 million of the 200 million curies in the salt waste is due to the presence of ${ }^{137} \mathrm{Cs}$ and daughter product ${ }^{137 m} \mathrm{Ba}$. The salt component consists of solid salt cake (16.5 million gallons) and supernate (17.5 million gallons). Currently, conversion of HLW sludge to glass by vitrification in the DWPF is underway. To date, approximately 30 million Curies of the original 226 million Curies present in the sludge component has been immobilized by vitrification. ${ }^{1}$

The original plan for the dispositioning of SRS HLW was to treat all salt cake waste (dissolved salt cake and supernate) in the Salt Waste Processing Facility SWPF. This approach is depicted schematically in Fig. 1 (ref. 2). The purpose of SWPF is to allow the bulk of the stored waste to be disposed of as low-level waste by segregating radionuclide constituents and transferring them to DWPF for vitrification with other HLW streams. In the SWPF, actinides and strontium are sorbed onto MST, which is then filtered from the waste stream. The solid/sludge stream from the sorption process is mixed with stored HLW sludge and immobilized as glass in the DWPF. The filtrate from the MST process is transferred to the SWPF CSSX process, in which cesium is selectively transferred into an organic extractant and is then back-extracted into a dilute nitric acid solution. In the SWPF CSSX flowsheet, flow parameters are set and mass transfer stages are configured to provide a decontamination factor (DF) of $>40,000$ (the cesium concentration in the feed is 40,000 times the concentration in the treated effluent) and a concentration factor of 15 (the cesium concentration in the aqueous back-extraction product is 15 times the level in the untreated feed). This concentrated Cs stream is to be transferred to the DWPF for vitrification. The deployment approach under the original plan was to design, construct, and operate a demonstration SWPF facility at $15 \%$ of the full-scale plant throughput, followed by deployment of a full-scale facility having a nominal annual capacity of 6 million gallons. 


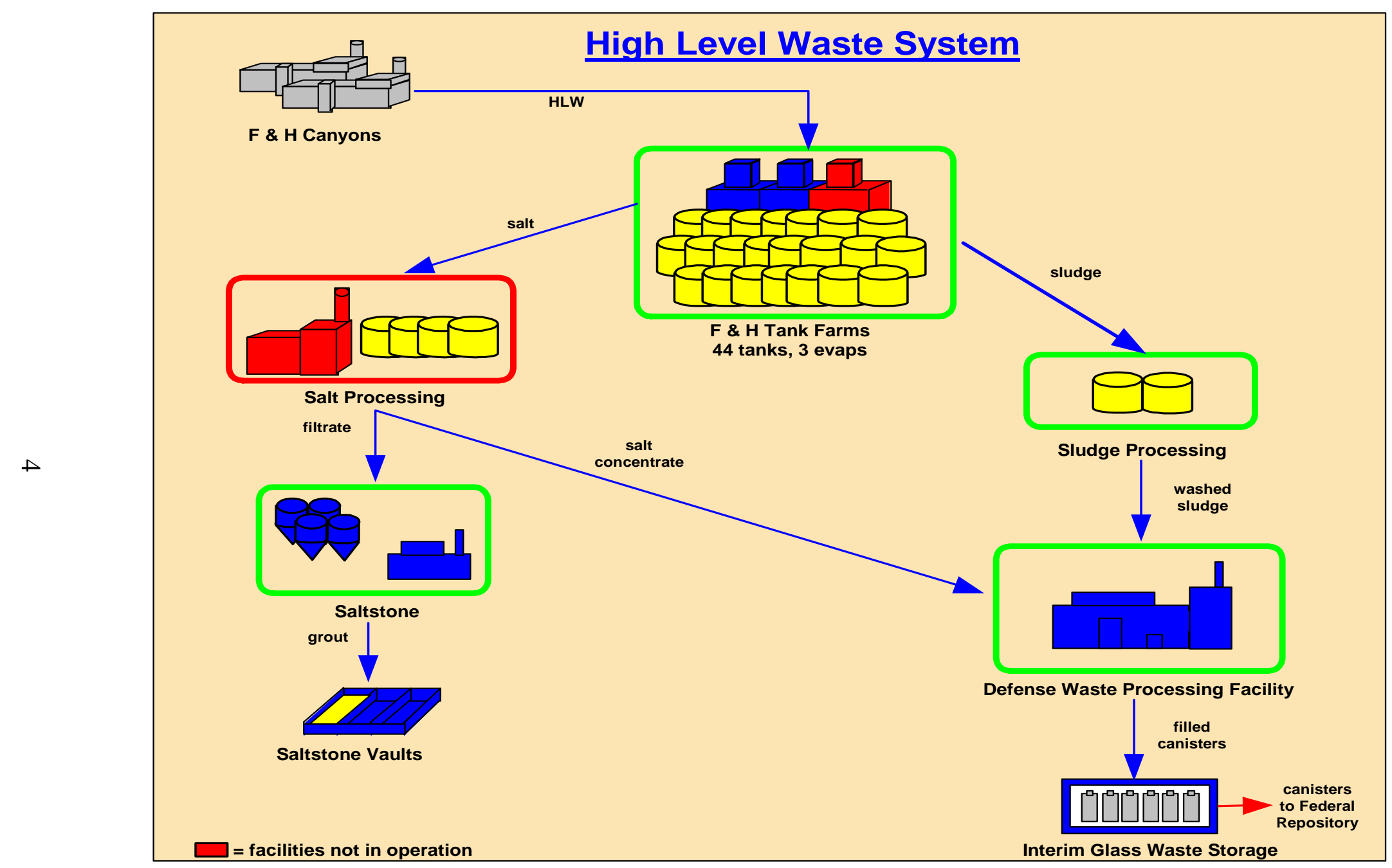

Fig. 1. Overview of SRS HLW process. 


\subsection{HLW SYSTEM DESCRIPTION}

The F-Area Tank Farm contains 22 waste tanks; 29 waste tanks are located in the H-Area Tank Farm. Tanks in F- and H- Area Tank Farms are of five different designs, which have evolved to reflect operating experience and technology advancements. While some differences in construction and support systems exist among the various designs (e.g., cooling and secondary containment), the basic concepts on which the designs were based are the same. Both tank farms also contain evaporator systems for waste volume reduction. Two evaporators, $1 \mathrm{~F}$ and $2 \mathrm{~F}$, are located in the F-Area Tank Farm. The 1F evaporator has been retired; the $2 \mathrm{~F}$ evaporator remains in operation. The H-Area Tank Farm contains three evaporators. The $1 \mathrm{H}$ evaporator has been retired; the $2 \mathrm{H}$ and $3 \mathrm{H}$ evaporators are currently operating. ${ }^{3}$

The Waste Transfer System facilitates intertank and interarea transfers within the waste management complex. To achieve transfer flexibility without an extensive piping network, transfer lines are routed to centrally located diversion boxes in which jumpers can be configured to accommodate specific transfer paths. Transfers between virtually any F- or H-Area tank to any other F- or H-Area tank or facility are made possible by reconfiguring jumpers in one or more of the 14 diversion boxes in the Waste Transfer System.

Processing of waste sludges begins with the Extended Sludge Process (ESP), which removes soluble salts from sludge prior to vitrification. The process begins with transfer of from 600,000 to 900,000 gal of sludge and salt waste to one of the ESP processing tanks. (HLW Tanks 40 and 51 are used in ESP.) Approximately 200,000 gal of water is added to the waste, and the contents are agitated for 5 to 8 days to ensure thorough mixing. After a 17- to 25-day settling period, the supernate is removed from the tank and sent to the $2 \mathrm{H}$ evaporator, from which it is transferred to a holding tank pending further processing. An additional wash water volume is added to the processing tank, and the agitation and settling procedures are repeated. From 8 to 12 wash cycles may be performed on a waste batch, depending on analysis of the sludge. Washed sludge is transferred to the DWPF for immobilization as glass. 
The first step in the vitrification process is to adjust the $\mathrm{pH}$ of the waste to meet feed specifications for the DWPF melter. The $\mathrm{pH}$ is adjusted by the addition of nitric acid, after which the slurry is boiled to remove mercury. The concentrated mixture is transferred to a tank, where a glass frit is added. After mixing the waste and frit into a slurry, the slurry is transferred to the melter, which produces molten glass. The melt is poured into stainless steel containers, which are sealed and decontaminated prior to transfer to the Glass Waste Storage Building for temporary storage.

Dissolved salt cake waste that meets the acceptance criteria for immobilization as grout is transferred from the tank farms to the Saltstone Manufacturing and Disposal Facility (SMDF). In SMDF the salt solution and the solid grout constituents are blended in the saltstone mixer in approximately a $40 \%$ solution $/ 60 \%$ solids proportion. The mixer discharges the blend into a hold tank while it awaits transfer. From the hold tanks, the blend is moved via grout transfer pumps into storage vaults, in which the blend is allowed to harden. The grout mixture remains free-flowing as long as it is kept in motion. When stationary, the mixture thickens significantly within $5 \mathrm{~min}$, and becomes rigidly solid within 2 days.

\subsection{CAUSTIC-SIDE SOLVENT EXTRACTION PROCESS}

The CSSX process was developed specifically for the extraction of cesium from alkaline supernate and dissolved salt cake wastes stored in the SRS HLW system. The key aspect of CSSX process development was the formulation of a solvent providing favorable cesium extraction and stripping (back-extraction) performance, chemical stability, acceptable phase-separation performance when dispersed with aqueous process streams, high cesium selectivity, and economical cleanup (washing to remove degradation products and other minor species). The primary component in the solvent, the extractant, is calix[4]arene-bis(tert-octylbenzo-crown-6), sometimes referred to as BOBCalixC6. ${ }^{4}$ This compound is a member of the calix[4]arene-crown- 6 family of extractants, which are hybrids of the calixarene and crown ether families. In these hybrids, one or two crown polyether chains form a bridge around the opposite phenolic positions of the calixarene in the 1-3-alt configuration. The facing pairs of aromatic rings

in the calixarene form cavities that are available for partial insertion of a cesium ion. Due 
to the preorganized configuration and a strong pi interaction between the aromatic rings and the cesium ion, the extractant has both high affinity and selectivity for cesium. Of special significance in the SRS application, the calix[4]arene-crown-6 family exhibits cesium/sodium separation factors exceeding $10^{4}$.

Due to the limited solvating ability of Isopar L®, the alkane diluent that is added to the solvent for hydrometallurgical reasons (density adjustment and phase-separation enhancement), a modifier is used. During development of the CSSX technology, the alkylphenyl ether alcohol (2,2,3,3-tetrafluoropropoxy)-3-(4-sec-butylphenoxy)-2propanol (known as Cs-7SB) was developed and demonstrated to be effective for increasing calix counterion solubility in the diluent, for eliminating the risk of third-phase formation, and for maximizing the extraction power of the calixarene. It was also designed to be highly stable to degradation and to yield aqueous-soluble fragments if breakdown occurs.

The CSSX process cascade comprises four operations: cesium extraction, scrubbing of the loaded solvent to remove co-extracted sodium and potassium, stripping to transfer the cesium into an aqueous solution, and washing of the stripped solvent to remove any solvent degradation products. In extraction, the solvent is contacted with an aqueous alkaline stream containing cesium. The extractant binds the $\mathrm{Cs}^{+}$ion into its cavity, and the resulting cationic complex is ion paired with an available anion. From modeling studies, the anion is thought to be primarily nitrate and deprotonated modifier (the alkoxide form).

Following extraction, the loaded solvent is scrubbed by contact with a dilute nitric acid stream ( $0.05 \mathrm{M}$ in previously demonstrated flowsheets). Scrubbing removes coextracted cations, particularly sodium and potassium ions, from the solvent and also removes extracted counterions (nitrate and hydroxide equivalents). The latter is essential for achieving effective stripping of cesium from the extract in the stripping section of the cascade and for minimizing the salt content of the strip effluent. Due to the countercurrent flow arrangement of the cascade, the scrub solution effluent is combined with the aqueous feed stream to the extraction section of the process and exits the flowsheet as part of the aqueous raffinate. 
Stripping or back-extraction of the solvated cesium is accomplished by contacting the scrubbed extract with a very dilute $(0.001 M) \mathrm{HNO}_{3}$ solution. Due to the very low concentration of the nitrate counterion in the strip solution, the extraction equilibrium is shifted away from the solvated cesium nitrate complex, resulting in preferential transfer of cesium into the aqueous phase. The presence of trioctylamine (TOA) in the solvent serves to counteract the presence of lipophilic anionic impurities which interfere with the stripping of cesium from the solvent. The aqueous product is collected and transferred for immobilization. The stripped solvent is contacted with a dilute caustic solution ( 0.001 to $0.010 \mathrm{M} \mathrm{NaOH}$ ) to remove chemical degradation products from the solvent, mainly 4-sec butylphenol together with minor anionic species that remain after stripping.

\subsection{SOLVENT EXTRACTION EQUIPMENT}

The key equipment component of the proposed technology is the centrifugal solvent extraction contactor, typically referred to as a "centrifugal contactor," or simply a "contactor." Centrifugal contactors were developed at SRS specifically for the extraction of actinides from dissolved spent nuclear reactor fuel and evolved from the mixer-settler. Both the mixer-settler and the centrifugal contactor were developed as alternatives to the pulsed-column extractor, which was also originally developed for actinide recovery and purification. The centrifugal contactor is preferred in the subject application, as the short residence time of solutions in the device translates into a small overall solvent inventory, which reduces plant capital costs owing to the high cost of the solvent (primarily the calixarene component). In addition, the short residence time in the contactor cascade limits contact time between the solvent and radionuclide species, thereby minimizing degradation of the solvent due to radiolysis.

Like the mixer-settler, the centrifugal contactor utilizes mechanical mixing to disperse one immiscible phase within another to promote transfer of a solute between the phases. In the mixer-settler, the dispersion flows into a quiescent area at the bottom of the device, wherein the dispersion separates into its component phases by gravity settling. The centrifugal contactor utilizes centrifugal force for phase separation. In general, the immiscible liquids between which solute is transferred are introduced separately into an annular cylindrical gap between a stationary outer housing and a cylindrical rotor, as 
shown in Fig. 2. Shear forces placed on the liquids cause the formation of a dispersion as a result of Couette mixing, hence the reference to this area of the contactor as the Couette or mixing zone. From the mixing zone, the dispersion flows into the rotor through an axial opening on the rotor's bottom face. Once inside the rotor, the dispersion is separated by centrifugal force due to the difference in the densities of the two phases. The rotor is configured so that the applied centrifugal force supports an annular layer of liquid along the inside wall comprising the separated phases, with the heavier phase

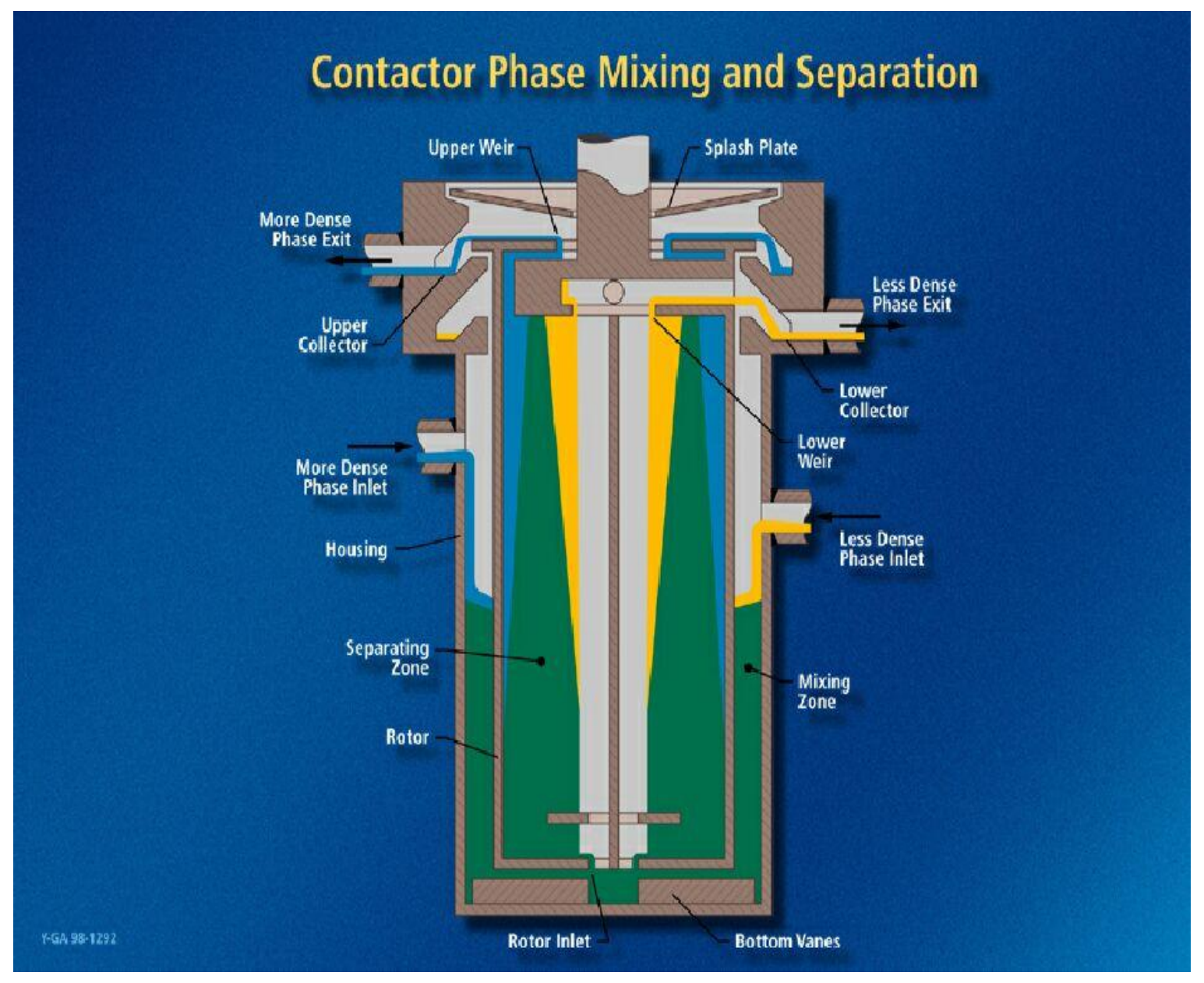

Fig. 2. Centrifugal contactor cross section with flow patterns. 
adjacent to the rotor wall. Passages machined at the top of the rotor direct the separated phases to discharge points at two different elevations, from which they are collected inside the housing. As is the case with mixer-settlers, centrifugal contactors are generally arrayed in multistage systems and are typically configured for countercurrent flow of the opposing liquid phases.

Centrifugal contactor cascades have been used successfully in actinide recovery operations for over 30 years. Stage efficiencies in these applications range from $90 \%$ to 98\% under normal operating conditions. Extensive laboratory- and bench-scale testing of contactors using CSSX solvent under extraction, scrubbing, stripping, and solvent washing conditions has been performed. The results of these tests have confirmed the technical feasibility of using a contactor-based cascade with CSSX solvent for cesium recovery from SRS HLW.

\section{PROCESS DESIGN}

\subsection{DESIGN CRITERIA}

The primary criteria from which the design was initiated pertain to system performance rather than to operation or maintenance. These criteria are as follows:

- Process 3 million gallons of dissolved salt cake annually, based on continuous operation and $90 \%$ system availability.

- Provide a DF capability of 40 (i.e., the concentration of ${ }^{137} \mathrm{Cs}$ in the treated effluent is to be one-fortieth the concentration in the untreated feed stream).

- Obtain a concentration factor (CF) of approximately 15 (i.e., the concentration of ${ }^{137} \mathrm{Cs}$ recovered in the aqueous effluent from the stripping section of the proposed simplified CSSX system, "CSSX-A," will be 15 times the concentration in the untreated feed stream).

Additional criteria that were established to facilitate system design are as follows: 
- Solvent, scrub, strip, and caustic wash solutions are to have the same compositions as those specified for the SWPF CSSX system.

- Solvent and scrub flow rates will be equal; therefore, the organic-to-aqueous (O-to-A) flow ratios in the scrubbing and stripping sections of the cascade will be equal.

Flow ratios applied in previous demonstrations of the SWPF CSSX system and in the current SWPF CSSX design were not retained in the CSSX-A system design. Instead, calculations were performed with the O-to-A flow ratio in the stripping section as a variable, and the effect on the number of extraction and stripping stages required to meet DF and CF criteria was determined. (Criteria for cesium CF and equal strip and scrub flows fix the O-to-A ratios in the extraction and scrubbing sections, once the ratio in the stripping section is selected.)

Additional criteria pertaining to system function were developed during the design. These include basic requirements for operation and maintenance of a contactorbased solvent extraction system. Requirements for processing of radioactive components have also been included.

- All effluent streams from the contactor system must flow by gravity into receiving vessels.

- Feed flows to the contactor system must be steady; use of positivedisplacement pumps or other fluid movers that deliver pulsing flows is to be avoided unless effective pulsation dampening is included.

- All equipment items are to be easily accessible for removal and replacement in the event of failure. 
- The system is to be designed for direct maintenance. Accordingly, provision has been included for draining and flushing the system and collecting system effluents in a shielded area separate from the main processing area.

- The system will have a dedicated heating, ventilation, and air conditioning (HVAC) system.

- The ventilation system will produce a negative gauge pressure in the operating area to prevent leakage of gases or vapors from the operating area to the outside environment. The ventilation system will be equipped with a high-efficiency particulate air (HEPA) filtration system.

- The areas of the process that contain radionuclides will be shielded so that the dose rate at the exterior surface of the process structure does not exceed $2.5 \mathrm{mrem} / \mathrm{h}$.

- The cost estimate will be based on the use of 300 series stainless steels or equivalent for all wetted components.

\subsection{PROCESS DESCRIPTION}

The primary component of the process is a cascade of centrifugal solvent extraction contactors in which cesium is selectively transferred from an aqueous caustic HLW solution into the CSSX solvent. Use of the CSSX solvent for cesium extraction was demonstrated during CSSX development activities that were performed to support selection of a cesium removal technology for deployment at SRS. These tests included distribution ratio determinations, single- and multi-stage mass transfer tests using a waste stream simulant, multi-stage mass transfer tests using SRS HLW (commonly referred to a "real waste tests"), chemical and thermal treatments and batch irradiation to evaluate solvent stability, and irradiation in a contactor test loop to evaluate the effect of radiolysis 
on phase separation. The majority of these tests were performed using the baseline CSSX solvent formulation, which is listed in Table 1.

Indications are that the original solvent composition is supersaturated with the extractant (BOBCalixC6) and that this component will precipitate from the solvent over time. To address this issue, a variety of alternative formulations were tested for solubility, cesium distribution behavior, and phase-separation performance. ${ }^{5}$ In addition, basic physical property data were collected for each formulation. The optimum composition determined after evaluating test results for candidate formulations is presented in the rightmost column in Table 1. The structures of the solvent components are presented in Fig. 3.

Basic flowsheets for the proposed simplified system (CSSX-A) and the SWPF CSSX system are shown in Figs. 4 and 5, respectively. The most significant difference between the two flowsheets is in the number of extraction stages. The difference is due primarily to the reduced DF performance requirement for the CSSX-A system (DF of 40 versus DF of 40,000 for the SWPF CSSX flowsheet). In addition, the O-to-A flow ratios in the cascades differ somewhat. The O-to-A ratios specified for CSSX-A are predicted to provide the greatest operating robustness using the minimum number of extraction and stripping stages. The number of scrubbing stages in the CSSX-A design was fixed at two, since this is the minimum number of stages required to reduce the concentrations of counterions in the extract to levels that will permit effective stripping. Use of excessive scrubbing stages will necessitate addition of extraction stages, since cesium distribution under scrubbing conditions results in a shift in the cesium concentration gradient toward the extraction end of the cascade.

An additional difference between the processing concepts on which the two flowsheets are based is the use of temperature control for optimization of interphase cesium transfer. The SWPF CSSX flowsheet includes provisions for maintaining processing temperatures near $20^{\circ} \mathrm{C}$ in the extraction section of the cascade and $30^{\circ} \mathrm{C}$ in the stripping section to maximize cesium transfer in the desired "direction" in these sections of the flowsheet. The proposed CSSX-A system does not include cascade temperature control because this capability has not been demonstrated in systems of production-scale contactors, and accelerated CSSX-A deployment precludes pursuing 
Table 1. CSSX solvent composition

\begin{tabular}{lcc}
\hline \multicolumn{1}{c}{ Component } & $\begin{array}{c}\text { Baseline } \\
\text { solvent }\end{array}$ & $\begin{array}{c}\text { Optimized } \\
\text { solvent }\end{array}$ \\
\hline $\begin{array}{l}\text { Calix[4]arene-bis(tert-octylbenzo-crown-6) } \\
\text { (aka BOBCalixC6) }\end{array}$ & $0.01 \mathrm{M}$ & $0.007 \mathrm{M}$ \\
$\begin{array}{l}(2,2,3,3-T e t r a f l u o r o p r o p o x y)-3-(4-s e c- \\
\text { butylphenoxy)-2-propanol (aka Cs-7SB) }\end{array}$ & $0.50 \mathrm{M}$ & $0.75 \mathrm{M}$ \\
$\begin{array}{l}\text { Trioctylamine } \\
\text { Isopar@ L }\end{array}$ & $0.001 \mathrm{M}$ & $0.003 \mathrm{M}$ \\
\hline
\end{tabular}

temperature control as a development task. Compensation for lack of thermally optimized cesium transfer has been made by increasing the number of stages in the extraction and stripping sections of the cascade to achieve the design-basis DF.

Material balances were solved to meet the processing criteria of 3 million gallons/year of waste treated, based on $90 \%$ system utilization and availability. The stream designations in the material balances are shown in Fig. 6 and defined in Table 2. Material balance results are presented in Tables 3 through 5.

Determination of the number of stages required in the flowsheet was performed using the basic Kremser equation,

$$
y_{N}=\frac{\beta^{N}-1}{\beta-1}\left(D x-y_{o}\right)+y_{o},
$$

where $\beta$ is the extraction factor defined by the expression

$$
\beta=\frac{D E}{F},
$$

in which $D$ is the solute distribution ratio, $E$ is the solvent flow rate, and $F$ is the aqueous feed stream flow rate. 


\section{Cesium Extractant}

@ $0.007 M$

- Synthetically attractive

- Rejects sodium

- Stable to alkaline simulant
BOBCalixC6

Calix[4]arene-bis(tert-octylbenzo-crown-6)

FW 1149.53

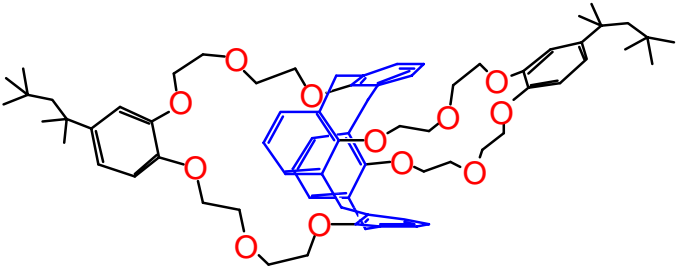

\section{Diluent Modifier}

@ $0.75 M$
Cs-7SB

1-(2,2,3,3-Tetrafluoropropoxy)-3-(4-secbutylphenoxy)-2-propanol

FW 338.35

- Increases Cs extraction

- Increases extractant solubility

- Excellent stability characteristics

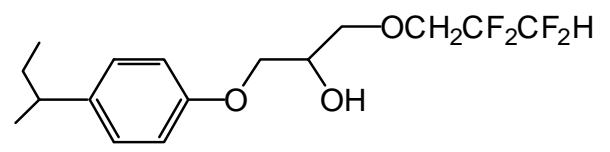

\section{Suppressor}

@ 0.003 M

- Suppresses impurity effects

- Suppresses ion-pair dissociation

- Improves and stabilizes stripping

\section{TOA}

Tri-n-octylamine

FW 353.69

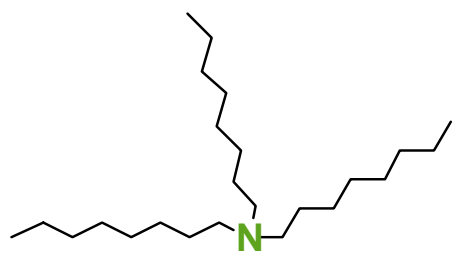

\section{Diluent}

- Isopar $^{\circledR} \mathrm{L}$

- Branched avg. 12-carbon aliphatic

- Promotes good hydraulics

Fig. 3. CSSX solvent components. 


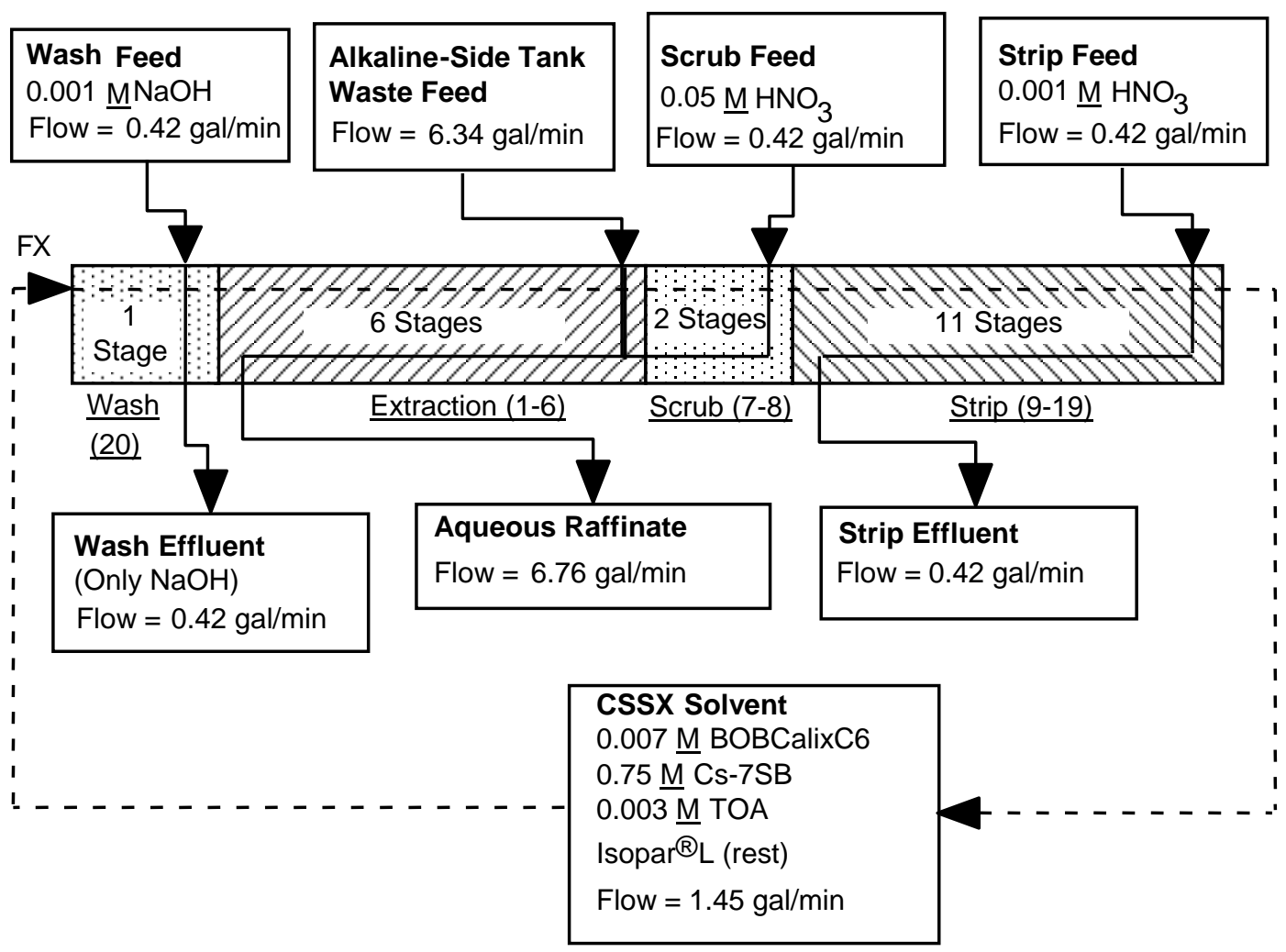

Fig. 4. Schematic of CSSX-A solvent extraction cascade.

The flowsheet was "solved" beginning with the stripping section of the cascade. This calculation required reversal of the nomenclature given in Eq. (1); that is, aqueous concentrations are designated by " $y$ " and organic concentrations by " $x$," and the extraction factor is the reciprocal of the value determined using Eq. (2). Given the desired concentration factor in the aqueous effluent from stripping and on the basis of a unit flow of waste feed to the extraction section, the stripping flow rate is fixed. The cesium concentration in the entering aqueous strip solution is zero, and the cesium concentration in the solvent after stripping was set at $1 \times 10^{-9} \mathrm{~mol} / \mathrm{L}$ to minimize cesium recycle to the extraction section of the cascade. The solvent-to-strip flow ratio was the 


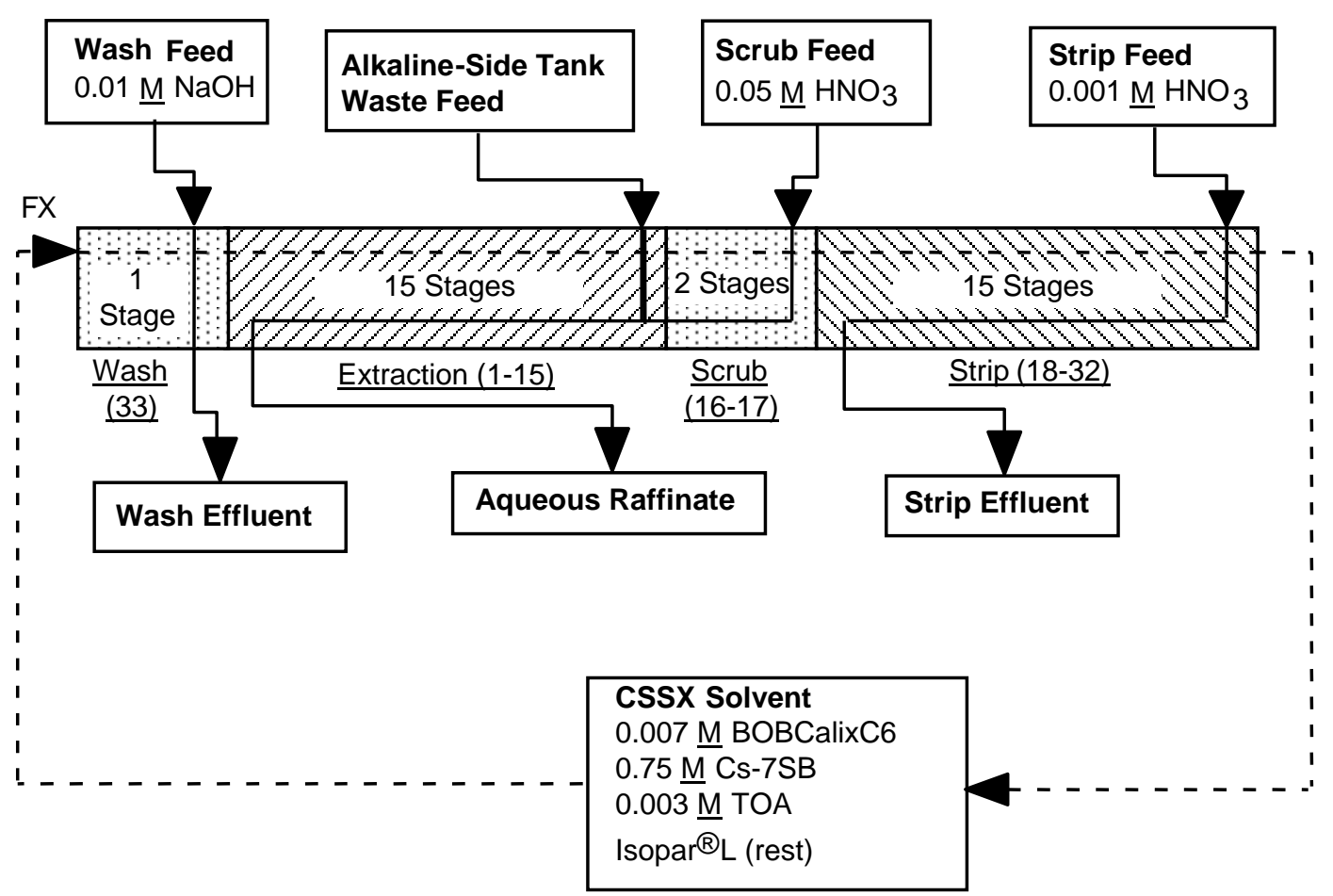

Fig. 5. Schematic of baseline CSSX flowsheet.

independent variable. With this one input, setting the scrub flow rate equal to the strip flow, fixing the number of scrubbing stages at 2 , and given a target cesium concentration in the aqueous raffinate based on the DF target, the number of stages in the extraction and stripping sections was determined.

Stage calculations for the extraction section of the cascade applied Eq. (1) using the standard nomenclature. All calculations used single distribution ratio values for the extraction and stripping conditions. Use of fixed $D_{C s}$ values for extraction and stripping is consistent with flowsheet modeling calculations performed as part of previous CSSX development and demonstration tasks. 


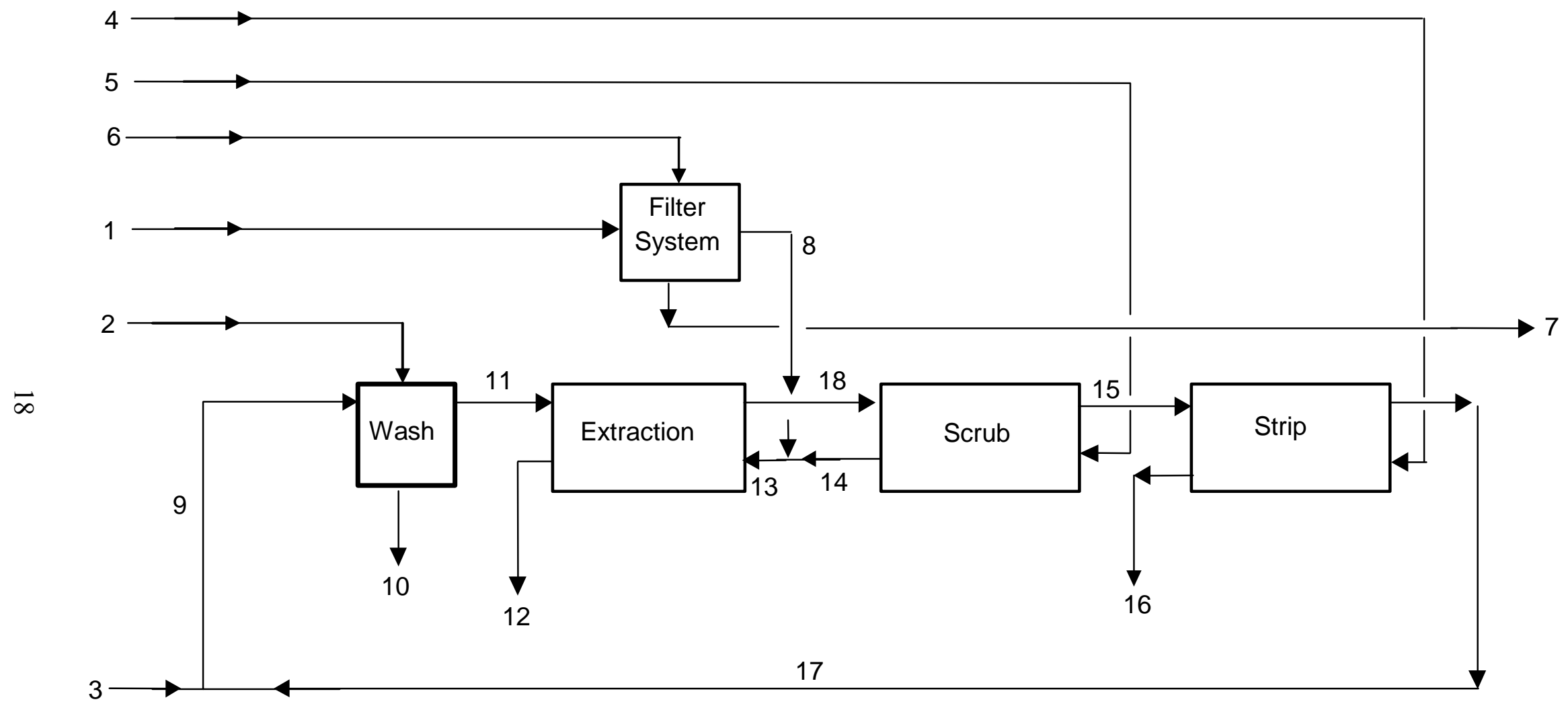

Fig. 6. Schematic of material balance flowsheet. 
Table 2. CSSX-A system stream descriptions ${ }^{a}$

\begin{tabular}{|c|l|}
\hline Stream & \\
\hline 1 & Aqueous feed stream from feed tank \\
\hline 2 & Feed stream for wash solution \\
\hline 3 & Makeup solvent line feeding solvent tank \\
\hline 4 & Feed stream for strip solution \\
\hline 5 & Feed stream for scrub solution \\
\hline 6 & Feed stream for filter backwash solution \\
\hline 7 & Recovered solids to DWPF for vitrification \\
\hline 8 & Feed filtrate to CSSX extraction contactor bank \\
\hline 9 & Stripped solvent to caustic washing \\
\hline 10 & Spent caustic wash solution to saltstone \\
\hline 11 & Washed solvent to CSSX extraction contactor bank \\
\hline 12 & Treated waste (raffinate from extraction) to saltstone for immobilization \\
\hline 13 & Combined aqueous feed stream and aqueous effluent from scrubbing contactors \\
\hline 14 & Aqueous effluent from scrubbing \\
\hline 15 & Scrubbed Cs-loaded solvent to stripping contactors \\
\hline 16 & Concentrated Cs-bearing aqueous effluent to DWPF for vitrification \\
\hline 18 & Stripped solvent \\
\hline
\end{tabular}

${ }^{a}$ Refer to Fig. 6 for schematic. 
Table 3. Material balance results, flows in kilogram moles per hour

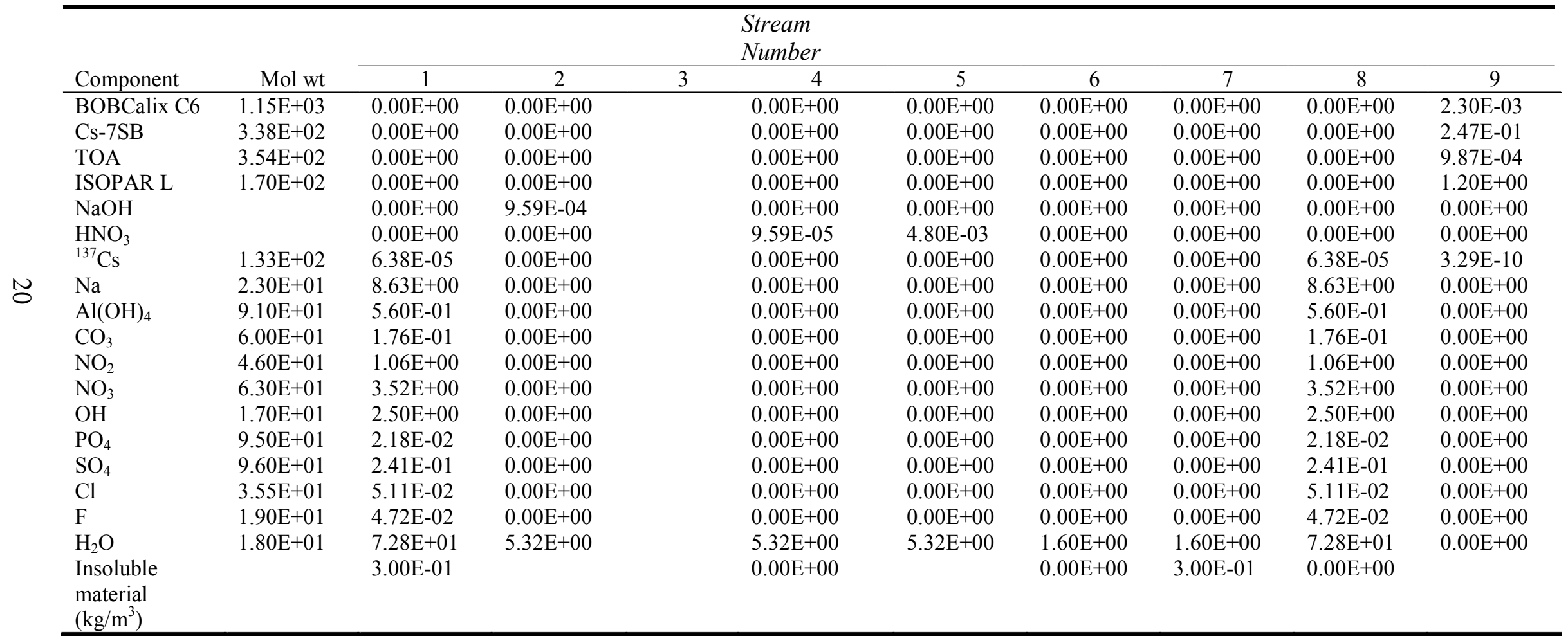


Table 3 (continued)

\begin{tabular}{|c|c|c|c|c|c|c|c|c|c|c|}
\hline \multicolumn{11}{|c|}{ Stream Number } \\
\hline Component & Mol wt & 10 & 11 & 12 & 13 & 14 & 15 & 16 & 17 & 18 \\
\hline BOBCalix C6 & $1.15 \mathrm{E}+03$ & $0.00 \mathrm{E}+00$ & $2.30 \mathrm{E}-03$ & $0.00 \mathrm{E}+00$ & $0.00 \mathrm{E}+00$ & $0.00 \mathrm{E}+00$ & $2.30 \mathrm{E}-03$ & $0.00 \mathrm{E}+00$ & $2.30 \mathrm{E}-03$ & $2.30 \mathrm{E}-03$ \\
\hline Cs-7SB & $3.38 \mathrm{E}+02$ & $0.00 \mathrm{E}+00$ & $2.47 \mathrm{E}-01$ & $0.00 \mathrm{E}+00$ & $0.00 \mathrm{E}+00$ & $0.00 \mathrm{E}+00$ & $2.47 \mathrm{E}-01$ & $0.00 \mathrm{E}+00$ & $2.47 \mathrm{E}-01$ & 2.47E-01 \\
\hline TOA & $3.54 \mathrm{E}+02$ & $0.00 \mathrm{E}+00$ & $9.87 \mathrm{E}-04$ & $0.00 \mathrm{E}+00$ & $0.00 \mathrm{E}+00$ & $0.00 \mathrm{E}+00$ & $9.87 \mathrm{E}-04$ & $0.00 \mathrm{E}+00$ & $9.87 \mathrm{E}-04$ & 9.87E-04 \\
\hline ISOPAR L & $1.70 \mathrm{E}+02$ & $0.00 \mathrm{E}+00$ & $1.20 \mathrm{E}+00$ & $0.00 \mathrm{E}+00$ & $0.00 \mathrm{E}+00$ & $0.00 \mathrm{E}+00$ & $1.20 \mathrm{E}+00$ & $0.00 \mathrm{E}+00$ & $1.20 \mathrm{E}+00$ & $1.20 \mathrm{E}+00$ \\
\hline $\mathrm{NaOH}$ & & $9.59 \mathrm{E}-04$ & $0.00 \mathrm{E}+00$ & $0.00 \mathrm{E}+00$ & $0.00 \mathrm{E}+00$ & $0.00 \mathrm{E}+00$ & $0.00 \mathrm{E}+00$ & $0.00 \mathrm{E}+00$ & $0.00 \mathrm{E}+00$ & $0.00 \mathrm{E}+00$ \\
\hline $\mathrm{HNO}_{3}$ & & & $0.00 \mathrm{E}+00$ & $4.80 \mathrm{E}-03$ & $4.80 \mathrm{E}-03$ & $4.80 \mathrm{E}-03$ & $0.00 \mathrm{E}+00$ & $9.59 \mathrm{E}-05$ & $0.00 \mathrm{E}+00$ & $0.00 \mathrm{E}+00$ \\
\hline${ }^{137} \mathrm{Cs}$ & $1.33 \mathrm{E}+02$ & & $3.29 \mathrm{E}-10$ & $1.59 \mathrm{E}-06$ & $6.38 \mathrm{E}-05$ & $0.00 \mathrm{E}+00$ & $6.22 \mathrm{E}-05$ & $6.22 \mathrm{E}-05$ & $3.29 \mathrm{E}-10$ & $6.22 \mathrm{E}-05$ \\
\hline $\mathrm{Na}$ & $2.30 \mathrm{E}+01$ & & $0.00 \mathrm{E}+00$ & $8.63 \mathrm{E}+00$ & $8.63 \mathrm{E}+00$ & $0.00 \mathrm{E}+00$ & $0.00 \mathrm{E}+00$ & $0.00 \mathrm{E}+00$ & $0.00 \mathrm{E}+00$ & $0.00 \mathrm{E}+00$ \\
\hline $\mathrm{Al}(\mathrm{OH})_{4}$ & $9.10 \mathrm{E}+01$ & & $0.00 \mathrm{E}+00$ & $5.60 \mathrm{E}-01$ & $5.60 \mathrm{E}-01$ & $0.00 \mathrm{E}+00$ & $0.00 \mathrm{E}+00$ & $0.00 \mathrm{E}+00$ & $0.00 \mathrm{E}+00$ & $0.00 \mathrm{E}+00$ \\
\hline $\mathrm{CO}_{3}$ & $6.00 \mathrm{E}+01$ & & $0.00 \mathrm{E}+00$ & $1.76 \mathrm{E}-01$ & $1.76 \mathrm{E}-01$ & $0.00 \mathrm{E}+00$ & $0.00 \mathrm{E}+00$ & $0.00 \mathrm{E}+00$ & $0.00 \mathrm{E}+00$ & $0.00 \mathrm{E}+00$ \\
\hline $\mathrm{NO}_{2}$ & $4.60 \mathrm{E}+01$ & & $0.00 \mathrm{E}+00$ & $1.06 \mathrm{E}+00$ & $1.06 \mathrm{E}+00$ & $0.00 \mathrm{E}+00$ & $0.00 \mathrm{E}+00$ & $0.00 \mathrm{E}+00$ & $0.00 \mathrm{E}+00$ & $0.00 \mathrm{E}+00$ \\
\hline $\mathrm{NO}_{3}$ & $6.30 \mathrm{E}+01$ & & $0.00 \mathrm{E}+00$ & $3.52 \mathrm{E}+00$ & $3.52 \mathrm{E}+00$ & $0.00 \mathrm{E}+00$ & $0.00 \mathrm{E}+00$ & $0.00 \mathrm{E}+00$ & $0.00 \mathrm{E}+00$ & $0.00 \mathrm{E}+00$ \\
\hline $\mathrm{OH}$ & $1.70 \mathrm{E}+01$ & & $0.00 \mathrm{E}+00$ & $2.50 \mathrm{E}+00$ & $2.50 \mathrm{E}+00$ & $0.00 \mathrm{E}+00$ & $0.00 \mathrm{E}+00$ & $0.00 \mathrm{E}+00$ & $0.00 \mathrm{E}+00$ & $0.00 \mathrm{E}+00$ \\
\hline $\mathrm{PO}_{4}$ & $9.50 \mathrm{E}+01$ & & $0.00 \mathrm{E}+00$ & $2.18 \mathrm{E}-02$ & $2.18 \mathrm{E}-02$ & $0.00 \mathrm{E}+00$ & $0.00 \mathrm{E}+00$ & $0.00 \mathrm{E}+00$ & $0.00 \mathrm{E}+00$ & $0.00 \mathrm{E}+00$ \\
\hline $\mathrm{SO}_{4}$ & $9.60 \mathrm{E}+01$ & & $0.00 \mathrm{E}+00$ & $2.41 \mathrm{E}-01$ & $2.41 \mathrm{E}-01$ & $0.00 \mathrm{E}+00$ & $0.00 \mathrm{E}+00$ & $0.00 \mathrm{E}+00$ & $0.00 \mathrm{E}+00$ & $0.00 \mathrm{E}+00$ \\
\hline $\mathrm{Cl}$ & $3.55 \mathrm{E}+01$ & & $0.00 \mathrm{E}+00$ & $5.11 \mathrm{E}-02$ & $5.11 \mathrm{E}-02$ & $0.00 \mathrm{E}+00$ & $0.00 \mathrm{E}+00$ & $0.00 \mathrm{E}+00$ & $0.00 \mathrm{E}+00$ & $0.00 \mathrm{E}+00$ \\
\hline $\mathrm{F}$ & $1.90 \mathrm{E}+01$ & & $0.00 \mathrm{E}+00$ & 4.72E-02 & 4.72E-02 & $0.00 \mathrm{E}+00$ & $0.00 \mathrm{E}+00$ & $0.00 \mathrm{E}+00$ & $0.00 \mathrm{E}+00$ & $0.00 \mathrm{E}+00$ \\
\hline $\mathrm{H}_{2} \mathrm{O}$ & $1.80 \mathrm{E}+01$ & $5.32 \mathrm{E}+00$ & $0.00 \mathrm{E}+00$ & $7.81 \mathrm{E}+01$ & $7.81 \mathrm{E}+01$ & $5.32 \mathrm{E}+00$ & $0.00 \mathrm{E}+00$ & $5.32 \mathrm{E}+00$ & $0.00 \mathrm{E}+00$ & $0.00 \mathrm{E}+00$ \\
\hline $\begin{array}{l}\text { Insoluble } \\
\text { material } \\
\left(\mathrm{kg} / \mathrm{m}^{3}\right)\end{array}$ & & & & & $0.00 \mathrm{E}+00$ & $0.00 \mathrm{E}+00$ & $0.00 \mathrm{E}+00$ & $0.00 \mathrm{E}+00$ & $0.00 \mathrm{E}+00$ & $0.00 \mathrm{E}+00$ \\
\hline
\end{tabular}


Table 4. Material balance results, flows in kilograms per hour

\begin{tabular}{|c|c|c|c|c|c|c|c|c|c|c|}
\hline \multicolumn{11}{|c|}{ Stream Number } \\
\hline Component & Mol wt & 1 & 2 & 3 & 4 & 5 & 6 & 7 & 8 & 9 \\
\hline BOBCalix C6 & $1.15 \mathrm{E}+03$ & & $0.00 \mathrm{E}+00$ & & & $0.00 \mathrm{E}+00$ & & $0.00 \mathrm{E}+00$ & $0.00 \mathrm{E}+00$ & $2.65 \mathrm{E}+00$ \\
\hline Cs-7SB & $3.38 \mathrm{E}+02$ & & $0.00 \mathrm{E}+00$ & & & $0.00 \mathrm{E}+00$ & & $0.00 \mathrm{E}+00$ & $0.00 \mathrm{E}+00$ & $8.35 \mathrm{E}+01$ \\
\hline TOA & $3.54 \mathrm{E}+02$ & & $0.00 \mathrm{E}+00$ & & & $0.00 \mathrm{E}+00$ & & $0.00 \mathrm{E}+00$ & $0.00 \mathrm{E}+00$ & $3.49 \mathrm{E}-01$ \\
\hline ISOPAR L & $1.70 \mathrm{E}+02$ & & $0.00 \mathrm{E}+00$ & & & $0.00 \mathrm{E}+00$ & & $0.00 \mathrm{E}+00$ & $0.00 \mathrm{E}+00$ & $2.03 \mathrm{E}+02$ \\
\hline $\mathrm{NaOH}$ & & & $3.84 \mathrm{E}-02$ & & & $0.00 \mathrm{E}+00$ & & $0.00 \mathrm{E}+00$ & $0.00 \mathrm{E}+00$ & $0.00 \mathrm{E}+00$ \\
\hline $\mathrm{HNO}_{3}$ & & & $0.00 \mathrm{E}+00$ & & $6.04 \mathrm{E}-03$ & $3.02 \mathrm{E}-01$ & & $0.00 \mathrm{E}+00$ & $0.00 \mathrm{E}+00$ & $0.00 \mathrm{E}+00$ \\
\hline${ }^{137} \mathrm{Cs}$ & $1.33 \mathrm{E}+02$ & $8.47 \mathrm{E}-03$ & $0.00 \mathrm{E}+00$ & $0.00 \mathrm{E}+00$ & $0.00 \mathrm{E}+00$ & $0.00 \mathrm{E}+00$ & $0.00 \mathrm{E}+00$ & $0.00 \mathrm{E}+00$ & $8.47 \mathrm{E}-03$ & $4.37 \mathrm{E}-08$ \\
\hline $\mathrm{Na}$ & $2.30 \mathrm{E}+01$ & $1.98 \mathrm{E}+02$ & $0.00 \mathrm{E}+00$ & $0.00 \mathrm{E}+00$ & $0.00 \mathrm{E}+00$ & $0.00 \mathrm{E}+00$ & $0.00 \mathrm{E}+00$ & $0.00 \mathrm{E}+00$ & $1.98 \mathrm{E}+02$ & $0.00 \mathrm{E}+00$ \\
\hline $\mathrm{Al}(\mathrm{OH})_{4}$ & $9.10 \mathrm{E}+01$ & $5.09 \mathrm{E}+01$ & $0.00 \mathrm{E}+00$ & $0.00 \mathrm{E}+00$ & $0.00 \mathrm{E}+00$ & $0.00 \mathrm{E}+00$ & $0.00 \mathrm{E}+00$ & $0.00 \mathrm{E}+00$ & $5.09 \mathrm{E}+01$ & $0.00 \mathrm{E}+00$ \\
\hline $\mathrm{CO}_{3}$ & $6.00 \mathrm{E}+01$ & $1.06 \mathrm{E}+01$ & $0.00 \mathrm{E}+00$ & $0.00 \mathrm{E}+00$ & $0.00 \mathrm{E}+00$ & $0.00 \mathrm{E}+00$ & $0.00 \mathrm{E}+00$ & $0.00 \mathrm{E}+00$ & $1.06 \mathrm{E}+01$ & $0.00 \mathrm{E}+00$ \\
\hline $\mathrm{NO}_{2}$ & $4.60 \mathrm{E}+01$ & $4.88 \mathrm{E}+01$ & $0.00 \mathrm{E}+00$ & $0.00 \mathrm{E}+00$ & $0.00 \mathrm{E}+00$ & $0.00 \mathrm{E}+00$ & $0.00 \mathrm{E}+00$ & $0.00 \mathrm{E}+00$ & $4.88 \mathrm{E}+01$ & $0.00 \mathrm{E}+00$ \\
\hline $\mathrm{NO}_{3}$ & $6.30 \mathrm{E}+01$ & $2.22 \mathrm{E}+02$ & $0.00 \mathrm{E}+00$ & $0.00 \mathrm{E}+00$ & $0.00 \mathrm{E}+00$ & $0.00 \mathrm{E}+00$ & $0.00 \mathrm{E}+00$ & $0.00 \mathrm{E}+00$ & $2.22 \mathrm{E}+02$ & $0.00 \mathrm{E}+00$ \\
\hline $\mathrm{OH}$ & $1.70 \mathrm{E}+01$ & $4.24 \mathrm{E}+01$ & $0.00 \mathrm{E}+00$ & $0.00 \mathrm{E}+00$ & $0.00 \mathrm{E}+00$ & $0.00 \mathrm{E}+00$ & $0.00 \mathrm{E}+00$ & $0.00 \mathrm{E}+00$ & $4.24 \mathrm{E}+01$ & $0.00 \mathrm{E}+00$ \\
\hline $\mathrm{PO}_{4}$ & $9.50 \mathrm{E}+01$ & $2.07 \mathrm{E}+00$ & $0.00 \mathrm{E}+00$ & $0.00 \mathrm{E}+00$ & $0.00 \mathrm{E}+00$ & $0.00 \mathrm{E}+00$ & $0.00 \mathrm{E}+00$ & $0.00 \mathrm{E}+00$ & $2.07 \mathrm{E}+00$ & $0.00 \mathrm{E}+00$ \\
\hline $\mathrm{SO}_{4}$ & $9.60 \mathrm{E}+01$ & $2.31 \mathrm{E}+01$ & $0.00 \mathrm{E}+00$ & $0.00 \mathrm{E}+00$ & $0.00 \mathrm{E}+00$ & $0.00 \mathrm{E}+00$ & $0.00 \mathrm{E}+00$ & $0.00 \mathrm{E}+00$ & $2.31 \mathrm{E}+01$ & $0.00 \mathrm{E}+00$ \\
\hline $\mathrm{Cl}$ & $3.55 \mathrm{E}+01$ & $1.81 \mathrm{E}+00$ & $0.00 \mathrm{E}+00$ & $0.00 \mathrm{E}+00$ & $0.00 \mathrm{E}+00$ & $0.00 \mathrm{E}+00$ & $0.00 \mathrm{E}+00$ & $0.00 \mathrm{E}+00$ & $1.81 \mathrm{E}+00$ & $0.00 \mathrm{E}+00$ \\
\hline $\mathrm{F}$ & $1.90 \mathrm{E}+01$ & $8.96 \mathrm{E}-01$ & $0.00 \mathrm{E}+00$ & $0.00 \mathrm{E}+00$ & $0.00 \mathrm{E}+00$ & $0.00 \mathrm{E}+00$ & $0.00 \mathrm{E}+00$ & $0.00 \mathrm{E}+00$ & $8.96 \mathrm{E}-01$ & $0.00 \mathrm{E}+00$ \\
\hline $\mathrm{H}_{2} \mathrm{O}$ & $1.80 \mathrm{E}+01$ & $1.31 \mathrm{E}+03$ & $9.59 \mathrm{E}+01$ & $0.00 \mathrm{E}+00$ & $9.59 \mathrm{E}+01$ & $9.59 \mathrm{E}+01$ & $2.88 \mathrm{E}+01$ & $2.88 \mathrm{E}+01$ & $1.31 \mathrm{E}+03$ & $0.00 \mathrm{E}+00$ \\
\hline $\begin{array}{l}\text { Insoluble } \\
\text { material } \\
\left(\mathrm{kg} / \mathrm{m}^{3}\right)\end{array}$ & & $3.00 \mathrm{E}-01$ & $0.00 \mathrm{E}+00$ & $0.00 \mathrm{E}+00$ & $0.00 \mathrm{E}+00$ & $0.00 \mathrm{E}+00$ & $0.00 \mathrm{E}+00$ & $3.00 \mathrm{E}-01$ & $0.00 \mathrm{E}+00$ & $0.00 \mathrm{E}+00$ \\
\hline $\operatorname{Sum}(\mathrm{kg} / \mathrm{h})$ & & $1.91 \mathrm{E}+03$ & $9.60 \mathrm{E}+01$ & $0.00 \mathrm{E}+00$ & $9.59 \mathrm{E}+01$ & $9.62 \mathrm{E}+01$ & $2.88 \mathrm{E}+01$ & $2.91 \mathrm{E}+01$ & $1.91 \mathrm{E}+03$ & $2.90 \mathrm{E}+02$ \\
\hline
\end{tabular}


Table 4 (continued)

\begin{tabular}{|c|c|c|c|c|c|c|c|c|c|c|}
\hline \multicolumn{11}{|c|}{ Stream Number } \\
\hline Component & Mol wt & 10 & 11 & 12 & 13 & 14 & 15 & 16 & 17 & 18 \\
\hline BOBCalix C6 & $1.15 \mathrm{E}+03$ & $0.00 \mathrm{E}+00$ & $2.65 \mathrm{E}+00$ & $0.00 \mathrm{E}+00$ & $0.00 \mathrm{E}+00$ & $0.00 \mathrm{E}+00$ & $2.65 \mathrm{E}+00$ & $0.00 \mathrm{E}+00$ & $2.65 \mathrm{E}+00$ & $2.65 \mathrm{E}+00$ \\
\hline Cs-7SB & $3.38 \mathrm{E}+02$ & $0.00 \mathrm{E}+00$ & $8.35 \mathrm{E}+01$ & $0.00 \mathrm{E}+00$ & $0.00 \mathrm{E}+00$ & $0.00 \mathrm{E}+00$ & $8.35 \mathrm{E}+01$ & $0.00 \mathrm{E}+00$ & $8.35 \mathrm{E}+01$ & $8.35 \mathrm{E}+01$ \\
\hline TOA & $3.54 \mathrm{E}+02$ & $0.00 \mathrm{E}+00$ & $3.49 \mathrm{E}-01$ & $0.00 \mathrm{E}+00$ & $0.00 \mathrm{E}+00$ & $0.00 \mathrm{E}+00$ & $3.49 \mathrm{E}-01$ & $0.00 \mathrm{E}+00$ & $3.49 \mathrm{E}-01$ & $3.49 \mathrm{E}-01$ \\
\hline ISOPAR L & $1.70 \mathrm{E}+02$ & $0.00 \mathrm{E}+00$ & $2.03 \mathrm{E}+02$ & $0.00 \mathrm{E}+00$ & $0.00 \mathrm{E}+00$ & $0.00 \mathrm{E}+00$ & $2.03 E+02$ & $0.00 \mathrm{E}+00$ & $2.03 \mathrm{E}+02$ & $2.03 \mathrm{E}+02$ \\
\hline $\mathrm{NaOH}$ & $5.84 \mathrm{E}+01$ & $3.84 \mathrm{E}-02$ & $0.00 \mathrm{E}+00$ & $0.00 \mathrm{E}+00$ & $0.00 \mathrm{E}+00$ & $0.00 \mathrm{E}+00$ & $0.00 \mathrm{E}+00$ & $0.00 \mathrm{E}+00$ & $0.00 \mathrm{E}+00$ & $0.00 \mathrm{E}+00$ \\
\hline $\mathrm{HNO}_{3}$ & $6.30 \mathrm{E}+01$ & $0.00 \mathrm{E}+00$ & $0.00 \mathrm{E}+00$ & $3.02 \mathrm{E}-01$ & $3.02 \mathrm{E}-01$ & $3.02 \mathrm{E}-01$ & $0.00 \mathrm{E}+00$ & $6.04 \mathrm{E}-03$ & $0.00 \mathrm{E}+00$ & $0.00 \mathrm{E}+00$ \\
\hline${ }^{137} \mathrm{Cs}$ & $1.33 \mathrm{E}+02$ & $0.00 \mathrm{E}+00$ & 4.37E-08 & $0.00 \mathrm{E}+00$ & $8.47 \mathrm{E}-03$ & $0.00 \mathrm{E}+00$ & $8.26 \mathrm{E}-03$ & $8.26 \mathrm{E}-03$ & $4.37 \mathrm{E}-08$ & $8.26 \mathrm{E}-03$ \\
\hline $\mathrm{Na}$ & $2.30 \mathrm{E}+01$ & $0.00 \mathrm{E}+00$ & $0.00 \mathrm{E}+00$ & $1.98 \mathrm{E}+02$ & $1.98 \mathrm{E}+02$ & $0.00 \mathrm{E}+00$ & $0.00 \mathrm{E}+00$ & $0.00 \mathrm{E}+00$ & $0.00 \mathrm{E}+00$ & $0.00 \mathrm{E}+00$ \\
\hline $\mathrm{Al}(\mathrm{OH})_{4}$ & $9.10 \mathrm{E}+01$ & $0.00 \mathrm{E}+00$ & $0.00 \mathrm{E}+00$ & $5.09 \mathrm{E}+01$ & $5.09 \mathrm{E}+01$ & $0.00 \mathrm{E}+00$ & $0.00 \mathrm{E}+00$ & $0.00 \mathrm{E}+00$ & $0.00 \mathrm{E}+00$ & $0.00 \mathrm{E}+00$ \\
\hline $\mathrm{CO}_{3}$ & $6.00 \mathrm{E}+01$ & $0.00 \mathrm{E}+00$ & $0.00 \mathrm{E}+00$ & $1.06 \mathrm{E}+01$ & $1.06 \mathrm{E}+01$ & $0.00 \mathrm{E}+00$ & $0.00 \mathrm{E}+00$ & $0.00 \mathrm{E}+00$ & $0.00 \mathrm{E}+00$ & $0.00 \mathrm{E}+00$ \\
\hline $\mathrm{NO}_{2}$ & $4.60 \mathrm{E}+01$ & $0.00 \mathrm{E}+00$ & $0.00 \mathrm{E}+00$ & $4.88 \mathrm{E}+01$ & $4.88 \mathrm{E}+01$ & $0.00 \mathrm{E}+00$ & $0.00 \mathrm{E}+00$ & $0.00 \mathrm{E}+00$ & $0.00 \mathrm{E}+00$ & $0.00 \mathrm{E}+00$ \\
\hline $\mathrm{NO}_{3}$ & $6.30 \mathrm{E}+01$ & $0.00 \mathrm{E}+00$ & $0.00 \mathrm{E}+00$ & $2.22 \mathrm{E}+02$ & $2.22 \mathrm{E}+02$ & $0.00 \mathrm{E}+00$ & $0.00 \mathrm{E}+00$ & $0.00 \mathrm{E}+00$ & $0.00 \mathrm{E}+00$ & $0.00 \mathrm{E}+00$ \\
\hline $\mathrm{OH}$ & $1.70 \mathrm{E}+01$ & $0.00 \mathrm{E}+00$ & $0.00 \mathrm{E}+00$ & $4.24 \mathrm{E}+01$ & $4.24 \mathrm{E}+01$ & $0.00 \mathrm{E}+00$ & $0.00 \mathrm{E}+00$ & $0.00 \mathrm{E}+00$ & $0.00 \mathrm{E}+00$ & $0.00 \mathrm{E}+00$ \\
\hline $\mathrm{PO}_{4}$ & $9.50 \mathrm{E}+01$ & $0.00 \mathrm{E}+00$ & $0.00 \mathrm{E}+00$ & $2.07 \mathrm{E}+00$ & $2.07 \mathrm{E}+00$ & $0.00 \mathrm{E}+00$ & $0.00 \mathrm{E}+00$ & $0.00 \mathrm{E}+00$ & $0.00 \mathrm{E}+00$ & $0.00 \mathrm{E}+00$ \\
\hline $\mathrm{SO}_{4}$ & $9.60 \mathrm{E}+01$ & $0.00 \mathrm{E}+00$ & $0.00 \mathrm{E}+00$ & $2.31 \mathrm{E}+01$ & $2.31 \mathrm{E}+01$ & $0.00 \mathrm{E}+00$ & $0.00 \mathrm{E}+00$ & $0.00 \mathrm{E}+00$ & $0.00 \mathrm{E}+00$ & $0.00 \mathrm{E}+00$ \\
\hline $\mathrm{Cl}$ & $3.55 \mathrm{E}+01$ & $0.00 \mathrm{E}+00$ & $0.00 \mathrm{E}+00$ & $1.81 \mathrm{E}+00$ & $1.81 \mathrm{E}+00$ & $0.00 \mathrm{E}+00$ & $0.00 \mathrm{E}+00$ & $0.00 \mathrm{E}+00$ & $0.00 \mathrm{E}+00$ & $0.00 \mathrm{E}+00$ \\
\hline $\mathrm{F}$ & $1.90 \mathrm{E}+01$ & $0.00 \mathrm{E}+00$ & $0.00 \mathrm{E}+00$ & $8.96 \mathrm{E}-01$ & $8.96 \mathrm{E}-01$ & $0.00 \mathrm{E}+00$ & $0.00 \mathrm{E}+00$ & $0.00 \mathrm{E}+00$ & $0.00 \mathrm{E}+00$ & $0.00 \mathrm{E}+00$ \\
\hline $\mathrm{H}_{2} \mathrm{O}$ & $1.80 \mathrm{E}+01$ & $9.59 \mathrm{E}+01$ & $0.00 \mathrm{E}+00$ & $1.41 \mathrm{E}+03$ & $1.41 \mathrm{E}+03$ & $9.59 \mathrm{E}+01$ & $0.00 \mathrm{E}+00$ & $9.59 \mathrm{E}+01$ & $0.00 \mathrm{E}+00$ & $0.00 \mathrm{E}+00$ \\
\hline $\begin{array}{l}\text { Insoluble } \\
\text { material } \\
\left(\mathrm{kg} / \mathrm{m}^{3}\right)\end{array}$ & - & $0.00 \mathrm{E}+00$ & $0.00 \mathrm{E}+00$ & $0.00 \mathrm{E}+00$ & $0.00 \mathrm{E}+00$ & $0.00 \mathrm{E}+00$ & $0.00 \mathrm{E}+00$ & $0.00 \mathrm{E}+00$ & $0.00 \mathrm{E}+00$ & $0.00 \mathrm{E}+00$ \\
\hline $\operatorname{Sum}(\mathrm{kg} / \mathrm{h})$ & - & $9.60 \mathrm{E}+01$ & $2.90 \mathrm{E}+02$ & $2.01 \mathrm{E}+03$ & $2.01 \mathrm{E}+03$ & $9.62 \mathrm{E}+01$ & $2.90 \mathrm{E}+02$ & $9.59 \mathrm{E}+01$ & $2.90 \mathrm{E}+02$ & $2.90 \mathrm{E}+02$ \\
\hline
\end{tabular}


Table 5. Material balance stream characterizations in kilogram moles per cubic meter

\begin{tabular}{|c|c|c|c|c|c|c|c|c|c|c|}
\hline \multicolumn{11}{|c|}{ Stream Number } \\
\hline Component & Mol wt & 1 & 2 & 3 & 4 & 5 & 6 & 7 & 8 & 9 \\
\hline BOBCalix C6 & $1.15 \mathrm{E}+03$ & - & - & $7.00 \mathrm{E}-03$ & - & - & - & - & $0.00 \mathrm{E}+00$ & $7.00 \mathrm{E}-03$ \\
\hline Cs-7SB & $3.38 \mathrm{E}+02$ & - & - & $7.50 \mathrm{E}-01$ & - & - & - & - & $0.00 \mathrm{E}+00$ & $7.50 \mathrm{E}-01$ \\
\hline TOA & $3.54 \mathrm{E}+02$ & - & - & $3.00 \mathrm{E}-03$ & - & - & - & - & $0.00 \mathrm{E}+00$ & $3.00 \mathrm{E}-03$ \\
\hline ISOPAR L & $1.70 \mathrm{E}+02$ & - & - & $3.64 \mathrm{E}+00$ & - & - & - & - & $0.00 \mathrm{E}+00$ & $3.64 \mathrm{E}+00$ \\
\hline $\mathrm{NaOH}$ & - & - & $1.00 \mathrm{E}-02$ & - & - & - & - & - & $0.00 \mathrm{E}+00$ & - \\
\hline $\mathrm{HNO}_{3}$ & - & - & - & - & $1.00 \mathrm{E}-03$ & $5.00 \mathrm{E}-02$ & 一 & - & $0.00 \mathrm{E}+00$ & - \\
\hline${ }^{137} \mathrm{Cs}$ & $1.33 \mathrm{E}+02$ & $4.43 \mathrm{E}-05$ & & - & $0.00 \mathrm{E}+00$ & $0.00 \mathrm{E}+00$ & - & - & $4.43 \mathrm{E}-05$ & $1.00 \mathrm{E}-09$ \\
\hline $\mathrm{Na}$ & $2.30 \mathrm{E}+01$ & $6.00 \mathrm{E}+00$ & - & - & - & - & 一 & - & $6.00 \mathrm{E}+00$ & - \\
\hline $\mathrm{Al}(\mathrm{OH})_{4}$ & $9.10 \mathrm{E}+01$ & $3.89 \mathrm{E}-01$ & - & - & - & - & - & - & $3.89 \mathrm{E}-01$ & - \\
\hline $\mathrm{CO}_{3}$ & $6.00 \mathrm{E}+01$ & $1.22 \mathrm{E}-01$ & - & - & - & - & - & - & $1.22 \mathrm{E}-01$ & - \\
\hline $\mathrm{NO}_{2}$ & $4.60 \mathrm{E}+01$ & $7.37 \mathrm{E}-01$ & - & - & - & - & - & - & 7.37E-01 & - \\
\hline $\mathrm{NO}_{3}$ & $6.30 \mathrm{E}+01$ & $2.45 \mathrm{E}+00$ & - & - & - & - & - & - & $2.45 \mathrm{E}+00$ & - \\
\hline $\mathrm{OH}$ & $1.70 \mathrm{E}+01$ & $1.73 \mathrm{E}+00$ & - & - & - & - & - & $0.00 \mathrm{E}+00$ & $1.73 \mathrm{E}+00$ & - \\
\hline $\mathrm{PO}_{4}$ & $9.50 \mathrm{E}+01$ & $1.51 \mathrm{E}-02$ & - & - & - & - & - & $0.00 \mathrm{E}+00$ & $1.51 \mathrm{E}-02$ & - \\
\hline $\mathrm{SO}_{4}$ & $9.60 \mathrm{E}+01$ & $1.67 \mathrm{E}-01$ & - & - & - & - & - & $0.00 \mathrm{E}+00$ & $1.67 \mathrm{E}-01$ & - \\
\hline $\mathrm{Cl}$ & $3.55 \mathrm{E}+01$ & $3.55 \mathrm{E}-02$ & 一 & - & - & - & - & $0.00 \mathrm{E}+00$ & $3.55 \mathrm{E}-02$ & - \\
\hline $\mathrm{F}$ & $1.90 \mathrm{E}+01$ & $3.28 \mathrm{E}-02$ & - & - & - & - & - & & $3.28 \mathrm{E}-02$ & - \\
\hline $\mathrm{H}_{2} \mathrm{O}$ & $1.80 \mathrm{E}+01$ & $5.06 \mathrm{E}+01$ & $5.55 \mathrm{E}+01$ & - & $5.55 \mathrm{E}+01$ & $5.55 \mathrm{E}+01$ & $5.55 \mathrm{E}+01$ & $5.55 \mathrm{E}+01$ & $5.06 \mathrm{E}+01$ & - \\
\hline $\begin{array}{l}\text { Insoluble } \\
\text { material } \\
\left(\mathrm{kg} / \mathrm{m}^{3}\right)\end{array}$ & - & $3.00 \mathrm{E}-01$ & - & - & - & - & - & $3.00 \mathrm{E}-01$ & $0.00 \mathrm{E}+00$ & - \\
\hline $\begin{array}{l}\text { Density } \\
\left(\mathrm{kg} / \mathrm{m}^{3}\right)\end{array}$ & - & $1.33 \mathrm{E}+03$ & $1.00 \mathrm{E}+03$ & $8.81 \mathrm{E}+02$ & $1.00 \mathrm{E}+03$ & $1.00 \mathrm{E}+03$ & $1.00 \mathrm{E}+03$ & $1.00 \mathrm{E}+03$ & $1.33 \mathrm{E}+03$ & $8.81 \mathrm{E}+02$ \\
\hline
\end{tabular}


Table 5 (continued)

\begin{tabular}{|c|c|c|c|c|c|c|c|c|c|c|}
\hline \multicolumn{11}{|c|}{ Stream Number } \\
\hline Component & Mol wt & 10 & 11 & 12 & 13 & 14 & 15 & 16 & 17 & 18 \\
\hline BOBCalix C6 & $1.15 \mathrm{E}+03$ & $0.00 \mathrm{E}+00$ & $7.00 \mathrm{E}-03$ & $0.00 \mathrm{E}+00$ & $0.00 \mathrm{E}+00$ & $0.00 \mathrm{E}+00$ & $7.00 \mathrm{E}-03$ & $0.00 \mathrm{E}+00$ & 7.00E-03 & $7.00 \mathrm{E}-03$ \\
\hline Cs-7SB & $3.38 \mathrm{E}+02$ & $0.00 \mathrm{E}+00$ & $7.50 \mathrm{E}-01$ & $0.00 \mathrm{E}+00$ & $0.00 \mathrm{E}+00$ & $0.00 \mathrm{E}+00$ & $7.50 \mathrm{E}-01$ & $0.00 \mathrm{E}+00$ & $7.50 \mathrm{E}-01$ & $7.50 \mathrm{E}-01$ \\
\hline TOA & $3.54 \mathrm{E}+02$ & $0.00 \mathrm{E}+00$ & $3.00 \mathrm{E}-03$ & $0.00 \mathrm{E}+00$ & $0.00 \mathrm{E}+00$ & $0.00 \mathrm{E}+00$ & $3.00 \mathrm{E}-03$ & $0.00 \mathrm{E}+00$ & $3.00 \mathrm{E}-03$ & $3.00 \mathrm{E}-03$ \\
\hline ISOPAR L & $1.70 \mathrm{E}+02$ & $0.00 \mathrm{E}+00$ & $3.64 \mathrm{E}+00$ & $0.00 \mathrm{E}+00$ & $0.00 \mathrm{E}+00$ & $0.00 \mathrm{E}+00$ & $3.64 \mathrm{E}+00$ & $0.00 \mathrm{E}+00$ & $3.64 \mathrm{E}+00$ & $3.64 \mathrm{E}+00$ \\
\hline $\mathrm{NaOH}$ & $5.84 \mathrm{E}+01$ & $1.00 \mathrm{E}-02$ & $0.00 \mathrm{E}+00$ & $0.00 \mathrm{E}+00$ & $0.00 \mathrm{E}+00$ & $0.00 \mathrm{E}+00$ & $0.00 \mathrm{E}+00$ & $0.00 \mathrm{E}+00$ & $0.00 \mathrm{E}+00$ & - \\
\hline $\mathrm{HNO}_{3}$ & $6.30 \mathrm{E}+01$ & $0.00 \mathrm{E}+00$ & $0.00 \mathrm{E}+00$ & $3.12 \mathrm{E}-03$ & $3.12 \mathrm{E}-03$ & $5.00 \mathrm{E}-02$ & $0.00 \mathrm{E}+00$ & $1.00 \mathrm{E}-03$ & $0.00 \mathrm{E}+00$ & - \\
\hline${ }^{137} \mathrm{Cs}$ & $1.33 \mathrm{E}+02$ & $0.00 \mathrm{E}+00$ & $1.00 \mathrm{E}-09$ & $1.04 \mathrm{E}-06$ & $4.15 \mathrm{E}-05$ & $0.00 \mathrm{E}+00$ & $1.89 \mathrm{E}-04$ & $6.48 \mathrm{E}-04$ & $1.00 \mathrm{E}-09$ & $1.89 \mathrm{E}-04$ \\
\hline $\mathrm{Na}$ & $2.30 \mathrm{E}+01$ & $0.00 \mathrm{E}+00$ & $0.00 \mathrm{E}+00$ & $5.62 \mathrm{E}+00$ & $5.62 \mathrm{E}+00$ & $0.00 \mathrm{E}+00$ & $0.00 \mathrm{E}+00$ & $0.00 \mathrm{E}+00$ & $0.00 \mathrm{E}+00$ & - \\
\hline $\mathrm{Al}(\mathrm{OH})_{4}$ & $9.10 \mathrm{E}+01$ & $0.00 \mathrm{E}+00$ & $0.00 \mathrm{E}+00$ & $3.65 \mathrm{E}-01$ & $3.65 \mathrm{E}-01$ & $0.00 \mathrm{E}+00$ & $0.00 \mathrm{E}+00$ & $0.00 \mathrm{E}+00$ & $0.00 \mathrm{E}+00$ & - \\
\hline $\mathrm{CO}_{3}$ & $6.00 \mathrm{E}+01$ & $0.00 \mathrm{E}+00$ & $0.00 \mathrm{E}+00$ & $1.15 \mathrm{E}-01$ & $1.15 \mathrm{E}-01$ & $0.00 \mathrm{E}+00$ & $0.00 \mathrm{E}+00$ & $0.00 \mathrm{E}+00$ & $0.00 \mathrm{E}+00$ & - \\
\hline $\mathrm{NO}_{2}$ & $4.60 \mathrm{E}+01$ & $0.00 \mathrm{E}+00$ & $0.00 \mathrm{E}+00$ & $6.91 \mathrm{E}-01$ & $6.91 \mathrm{E}-01$ & $0.00 \mathrm{E}+00$ & $0.00 \mathrm{E}+00$ & $0.00 \mathrm{E}+00$ & $0.00 \mathrm{E}+00$ & - \\
\hline $\mathrm{NO}_{3}$ & $6.30 \mathrm{E}+01$ & $0.00 \mathrm{E}+00$ & $0.00 \mathrm{E}+00$ & $2.29 \mathrm{E}+00$ & $2.29 \mathrm{E}+00$ & $0.00 \mathrm{E}+00$ & $0.00 \mathrm{E}+00$ & $0.00 \mathrm{E}+00$ & $0.00 \mathrm{E}+00$ & 一 \\
\hline $\mathrm{OH}$ & $1.70 \mathrm{E}+01$ & $0.00 \mathrm{E}+00$ & $0.00 \mathrm{E}+00$ & $1.63 \mathrm{E}+00$ & $1.63 \mathrm{E}+00$ & $0.00 \mathrm{E}+00$ & $0.00 \mathrm{E}+00$ & $0.00 \mathrm{E}+00$ & $0.00 \mathrm{E}+00$ & - \\
\hline $\mathrm{PO}_{4}$ & $9.50 \mathrm{E}+01$ & $0.00 \mathrm{E}+00$ & $0.00 \mathrm{E}+00$ & $1.42 \mathrm{E}-02$ & $1.42 \mathrm{E}-02$ & $0.00 \mathrm{E}+00$ & $0.00 \mathrm{E}+00$ & $0.00 \mathrm{E}+00$ & $0.00 \mathrm{E}+00$ & - \\
\hline $\mathrm{SO}_{4}$ & $9.60 \mathrm{E}+01$ & $0.00 \mathrm{E}+00$ & $0.00 \mathrm{E}+00$ & $1.57 \mathrm{E}-01$ & $1.57 \mathrm{E}-01$ & $0.00 \mathrm{E}+00$ & $0.00 \mathrm{E}+00$ & $0.00 \mathrm{E}+00$ & $0.00 \mathrm{E}+00$ & - \\
\hline $\mathrm{Cl}$ & $3.55 \mathrm{E}+01$ & $0.00 \mathrm{E}+00$ & $0.00 \mathrm{E}+00$ & $3.33 \mathrm{E}-02$ & $3.33 \mathrm{E}-02$ & $0.00 \mathrm{E}+00$ & $0.00 \mathrm{E}+00$ & $0.00 \mathrm{E}+00$ & $0.00 \mathrm{E}+00$ & - \\
\hline $\mathrm{F}$ & $1.90 \mathrm{E}+01$ & $0.00 \mathrm{E}+00$ & $0.00 \mathrm{E}+00$ & $3.07 \mathrm{E}-02$ & $3.07 \mathrm{E}-02$ & $0.00 \mathrm{E}+00$ & $0.00 \mathrm{E}+00$ & $0.00 \mathrm{E}+00$ & $0.00 \mathrm{E}+00$ & - \\
\hline $\mathrm{H}_{2} \mathrm{O}$ & $1.80 \mathrm{E}+01$ & $5.55 \mathrm{E}+01$ & $0.00 \mathrm{E}+00$ & $5.09 \mathrm{E}+01$ & $5.09 \mathrm{E}+01$ & $5.55 \mathrm{E}+01$ & $0.00 \mathrm{E}+00$ & $5.55 \mathrm{E}+01$ & $0.00 \mathrm{E}+00$ & - \\
\hline $\begin{array}{l}\text { Insoluble } \\
\text { material } \\
\left(\mathrm{kg} / \mathrm{m}^{3}\right)\end{array}$ & - & $0.00 \mathrm{E}+00$ & $0.00 \mathrm{E}+00$ & $0.00 \mathrm{E}+00$ & $0.00 \mathrm{E}+00$ & - & $0.00 \mathrm{E}+00$ & $0.00 \mathrm{E}+00$ & $0.00 \mathrm{E}+00$ & - \\
\hline $\begin{array}{l}\text { Density } \\
\left(\mathrm{kg} / \mathrm{m}^{3}\right)\end{array}$ & - & $1.00 \mathrm{E}+03$ & $8.81 \mathrm{E}+02$ & $0.00 \mathrm{E}+00$ & - & $1.00 \mathrm{E}+03$ & $8.81 \mathrm{E}+02$ & $1.00 \mathrm{E}+03$ & $8.81 \mathrm{E}+02$ & $8.81 \mathrm{E}+02$ \\
\hline
\end{tabular}


As stated previously, the distribution ratio under extraction conditions was predicted using a thermodynamic model that was verified previously against the original CSSX solvent composition. Prediction of a value was necessary in order to compensate for (1) a higher level of sodium $(6.44 M)$ than was present in simulated waste solutions used in previous equilibration and multi-stage flowsheet tests (5.6 $M)$ and (2) the combination of waste feed and scrub solutions in the extraction section of the cascade. The predicted value of $D_{C s}$ in extraction is 9.17 (ref. 6); a value of 9.0 was used in the extraction stage-calculations. The value used to determine the number of stripping stages was 0.08 , a somewhat conservative value that is based on experimental results reported for the optimized solvent. ${ }^{5}$ All calculations were based on system operation at $25^{\circ} \mathrm{C}$.

Cascade flow rates were determined from the stage number calculations and the material balance. Key flow values are shown in Fig. 4. As shown in Fig. 4, the cascade consists of 6 stages of extraction, 2 stages of scrubbing, 11 stages of stripping, and 1 solvent wash stage arranged for countercurrent flow of organic and aqueous streams. Numbering stages in order from extraction to stripping and solvent washing, the solvent enters Stage 1, from which the aqueous raffinate exits. The raffinate stream, which is the combination of treated waste and aqueous scrub solution, is transferred to a holding tank prior to transfer for immobilization as saltstone. The current design is based on transfer of this solution from the CSSX-A system to Tank 41, where it will be characterized to verify that it meets the Saltstone WAC prior to transfer to SMDF for immobilization. The solvent is delivered from the solvent feed tank, which holds a nominal inventory of 100 gal.

The waste feed stream enters the cascade at Stage 6 after passing through one of the two cross-flow filtration units and is combined with the aqueous effluent from the scrubbing section of the cascade. Feed is supplied from a low-residence-time feed tank that provides a buffer between the cascade and the HLW system. The purpose of providing a small feed-holdup capacity is to simplify system startup and to provide feed solution to allow for an orderly system shutdown in the event that feed delivery from the HLW system is interrupted. 
Due to the likely presence of high levels of cesium and actinides in the solids collected in the feed filters, the back-flush solution from the filters will be combined with the concentrated aqueous cesium product from the stripping section of the cascade and then transferred for vitrification in the DWPF.

The scrub solution, $0.05 \mathrm{MHNO}_{3}$, is delivered to the cascade at Stage 8. The strip solution, $0.001 \mathrm{MHNO}_{3}$, enters the cascade at Stage 19 and exits the cascade from Stage 9. Both scrub and strip solutions are delivered to the cascade from tanks located in a cold chemical makeup area that is separate from the shielded operating areas. Two scrub and two strip feed tanks are included to permit makeup of solutions without interrupting system operation. Due to the need to neutralize the product before transfer to the DWPF feed tank for holding prior to vitrification, two product tanks are installed in parallel, each having a nominal holdup time of at least $6 \mathrm{~h}$.

The stripped solvent is contacted with nominally $0.001 \mathrm{M} \mathrm{NaOH}$ in Stage 20 to remove any chemical degradation products prior to recycle of the solvent to the extraction section of the cascade. Due to the low level of cesium present in this stream, it will be combined with the raffinate from extraction and transferred to Tank 41 for eventual immobilization as saltstone. As is the case with scrub and strip solutions, the wash solution is delivered from one of two parallel tanks located in the cold chemical makeup area.

Two alternative disposal operations for spent solvent were identified during performance of the design project. Due to the low solvent disposal rate (estimated at 100 gal/year), it has been determined that spent solvent can be adsorbed onto "oil dry" and disposed of as solid waste. Alternatively, spent solvent can be drummed and transferred to the SWPF CSSX system for consolidation and disposal with spent solvent from that process. Given that projected CSSX-A deployment is expected to predate SWPF startup by 3-4 years, the solvent used in the CSSX-A system during this period will have to be stored until SWPF comes on-line.

Based on results from previous development activities, it is expected that some fraction of the solvent will be lost as entrainment in the raffinate and product streams from the process. If the proposed alternative technology is selected for deployment, determination of the rate of loss will be made as a prerequisite to detailed system design 
so that the loss rate can be mitigated by addition of separating equipment to the flowsheet, if necessary.

\subsection{EQUIPMENT DESCRIPTIONS}

\subsubsection{Centrifugal Contactors}

To estimate contactor costs and to development equipment layouts, contactor sizes were determined using a correlation developed for devices ranging in size from 4 to $25 \mathrm{~cm}$, where the size refers to the outside diameter of the rotor. The results of these calculations indicate that contactors having a nominal rotor diameter of $15 \mathrm{~cm}$ operating at maximum throughput are required in the extraction section of the cascade. At maximum capacity, contactors having 8-cm-diam rotors are required in the scrubbing, stripping, and solvent washing sections of the cascade.

Historically, contactors used in operations involving radionuclides have been designed and fabricated for each specific application. However, due to the desire to expedite deployment of the proposed technology, efforts have been made to locate commercially available process equipment, thereby minimizing or eliminating the time and cost associated with detailed design of process components. Within the last 10 15 years, DOE-owned contactor technology has been licensed by at least one commercial vendor [Costner Texas Industries (CTI)], which has developed standard contactor designs for a limited number of contactor sizes. To facilitate procurement of contactors of standard CTI designs, the extraction contactor size has been specified at 25-cm (10-in) rotor diameter; the scrubbing, stripping, and washing contactors have been specified at 12.5-cm (5-in) rotor diameter. The 25- and 12.5-cm units have maximum rated capacities of 30 and $5 \mathrm{gal} / \mathrm{min}$, respectively. It is important to note that the actual capacity of a centrifugal contactor is dependent on the phase-separation behavior of the particular system of interest and is also impacted by the phase ratio.

\subsubsection{Cross-flow Filters}

Experience with centrifugal contactors indicates that solids present in solutions fed to these devices are effectively captured and accumulate within the contactor rotors. 
This is particularly true of larger, higher-density solids. To address the likelihood that a minor fraction of solids will be carried to the CSSX-A system from upstream processes, cross-flow filters have been specified for installation in the aqueous feed stream to the extraction section of the cascade. For cost-estimation purposes, a HyPulse ${ }^{\circledR}$ LSI filtration system manufactured by the Mott Corporation has been selected for installation in the proposed system. The system consists of two filters installed in parallel, with each filter comprising a stainless steel housing containing seven filter elements graded at $0.5 \mu \mathrm{m}$. The filter grade is consistent with typical requirements for centrifugal contactor installations in which $99 \%$ removal of particles $1 \mu \mathrm{m}$ and larger is recommended. Because the filters are of the cross-flow type, a stream equivalent to $2 \%$ of the feed rate on a continuous basis is required for filter rinsing. Differential pressure sensors will be installed across each filter housing to provide indication of solids accumulation prior to loss of filter performance.

At the time this report was prepared, evaluations of the performance of rotary filters for clarification of HLW solutions were under way. Pending the results of this activity, it is likely that filters of this type will be established as the device of choice for application at SRS. Due to the relatively low cost of filter equipment, it is not anticipated that uncertainty regarding the type of filter used in CSSX-A will affect the CSSX-A project cost estimate or the cost/benefit analysis appreciably.

\subsubsection{Fluid Movers}

For cost-estimation purposes, canned rotor centrifugal pumps have been selected for use in the CSSX-A system. This selection was consistent with the original equipment layout concept in which all pumps were located below associated supply tanks. In the design, each fluid transfer application has been provided with two pumps installed in parallel, providing redundancy as mitigation against pump failure.

As the system design evolved, a second system configuration concept was developed in which pumps with suction lift capability are located above their respective supply tanks. The benefit of this alternative configuration is the ability to locate pumps, which contain higher-frequency-of-repair components, in a common enclosure (an operating gallery) with the contactor cascade instead of placing them in the enclosure 
containing feed, raffinate, and product tanks. This arrangement has advantages with regard both to accessibility for maintenance and pump longevity. Radiation dose rate in the operating gallery is reduced because of isolation from the bulk of the radionuclide inventory.

Due to the relatively high cost of canned rotor pumps, it is not expected that the change in pump selection to accommodate the modified configuration will result in increased system cost.

\subsubsection{Tankage}

Tank layouts and costs have been based on use of standard cylindrical vessels fabricated from 300 series low-carbon stainless steel. Due to the low levels of fissile materials expected to be present in the system, critically safe tank geometries are not required in the CSSX-A system.

\subsection{SYSTEM DEPLOYMENT}

Two general deployment concepts were considered from the outset of the project: installation of the process in an existing but unused SRS facility or deployment of the process in one or more transportable containers assembled as a new installation. Construction of a new bricks-and-mortar facility was not considered, as this approach is inherently unfavorable from both cost and schedule standpoints. Use of an existing facility has the potential advantage of accelerating deployment and eliminates cost associated with construction of a new processing building. The potentials for cost savings and schedule acceleration can be limited by the need to upgrade or renovate the existing structure, particularly if the facility is contaminated with radionuclides, which necessitate special access and waste disposal considerations.

The original skid-mounted containerized approach was based on installation of equipment inside two or more, standard-size, over-the-road trailers. These trailers with installed process equipment could be fabricated anywhere and transported to the SRS for integration into the HLW system. A second containerized concept that was developed during the project calls for skid-mounted equipment to be installed inside standard-size 
shipping containers of the type that are commonly used to ship materials on flatbed rail cars or on container ships.

Selection of the final deployment concept recommendation has been made based on several criteria:

- availability,

- configuration,

- availability of needed services,

- proximity to existing system and tank interfaces,

- existence of HLW system tie-ins, and

- cost (existing facility upgrades versus containers and ancillaries).

Due to the accelerated schedule imposed on the subject project, determination of costs required to upgrade existing facilities was not made; therefore, no cost comparison between deployment concepts was performed. Evaluation of the deployment options against the remaining criteria are discussed in the following sections.

\subsubsection{Building 241-96H}

Building 241-96H is well situated for the proposed activity, being located in the H-Area Tank Farm adjacent to the grouping of Tanks 48, 49, 50, and 51. Building 241$96 \mathrm{H}$ is actually two buildings, the Filter Building and the Stripper Building, the latter being an addition to the former. The Filter Building was constructed to house equipment for filtering solutions that were removed from HLW tanks following in-tank precipitation of cesium using tetraphenylborate (TPB). The facility has been in cold standby since intank precipitation processing was suspended due to safety concerns. The primary areas of interest in the proposed project are two shielded filter cells having identical asymmetric layouts. Each cell has an overall height of $17 \mathrm{ft} 6 \mathrm{in}$. The lower $6 \mathrm{ft} 6 \mathrm{in}$. of each pit is $15 \mathrm{ft} 6$ in. wide by $17 \mathrm{ft}$ long. The upper $11 \mathrm{ft}$ of each pit is $15 \mathrm{ft} 6$ in. wide by $24 \mathrm{ft}$ long. The pits are separated by a concrete wall that is $3 \mathrm{ft}$ thick. North of the filter cells is an unshielded area containing two filtrate tanks. 
Of the two existing facilities considered, this location is the most favorable. Location adjacent to Tanks $48-51$ is significant since Tank 51 is projected to receive the high-activity product stream from CSSX-A and Tank 48 is a candidate feed supply tank to the CSSX-A system. Space in either of the available filter cells is adequate for installation of the radionuclide-containing portion of the CSSX-A process. (The portion of the process requiring shielding consists of the contactor cascade; the feed, raffinate, product, and solvent tanks; and the associated pumps.) There are potential equipment access concerns if a single cell is used to house the shielded portion of the process, because tanks and pumps would have to be installed below the contactors, making replacement of the latter difficult. In addition, if a single cell is used for installation of CSSX-A, subdivision of the cell to separate pumps and contactors from the high-rad tank inventories is desirable.

Availability of the filter cells is not clear. Information received from project teams proposing complementary alternative processing technologies indicated that these projects intended to use the filter cells.

SRS HLW operating personnel contacted during the design process expressed a preference for the skid-mounted approach over installation of CSSX-A in Building 241$96 \mathrm{H}$.

\subsubsection{Building 512S}

Building 512S previously housed the Late Wash Facility. The primary function of this facility was to wash the precipitate from the In-Tank Precipitation Process prior to transfer of the material to DWPF for vitrification. In the Late Wash Facility, the precipitate was contacted with a solution to remove corrosion-inhibiting sodium nitrite from the salt solution that was pretreated at the In-Tank Precipitation Facility. From the wash tank, the precipitate and wash water were passed through a cross-flow filter to separate washed precipitate from contaminants and wash water. The precipitate was then pumped to the DWPF for immobilization as glass.

Deployment of CSSX-A in Building 512S was dropped from consideration based on evaluation against two factors: availability and system interface logistics. First, the CSSX-A project team was informed that Building 512S is to serve as the location for the 
Actinide Removal Process, which will receive low-curie salt that does not require processing in SWPF but does require removal of actinides and/or strontium in order to meet SMDF WAC. Second, Building 512S is located at lower elevation than Tank 51, which is to receive the high-activity cesium product stream from CSSX-A. Consequently, the transfer line from CSSX-A to Tank 51 could not be emptied completely without having to pressurize the transfer line. This is considered undesirable from a safety standpoint.

\subsubsection{Skid-Mounted Containerized System}

As mentioned previously, the original skid-mounted deployment concept called for installation of the CSSX-A system using interconnected, standard-size, over-the-road (semi) trailers. This approach has the advantage of mobility, at least until necessary shielding is installed. However, the presence of the trailer undercarriage (tires, axles, etc.) makes stacking of enclosures impossible, thus adding to the system footprint. During a presentation of the skid-mounted trailer concept as SRS, HLW personnel suggested that the project consider deployment of the system using shielded shipping containers. Subsequently, information was received from SRTC personnel, indicating that shielded shipping containers were commercially available. (Such containers had been constructed previously for shipment and storage of radioactive materials.)

The project team was able to locate a supplier of shipping containers that has the capability of making container modifications including addition of shielding and installation of access doors. Standard containers are available in $20-\mathrm{ft}, 40-\mathrm{ft}$, and $48-\mathrm{ft}$ lengths. Price quotes for standard (unmodified) containers are $\$ 2300$ for a 20 - $\mathrm{ft}$ unit, $\$ 2700$ for a 40 -ft unit, and $\$ 8800$ for a 48 -ft unit. No estimate for addition of shielding was obtained; access doors may be added at a cost of $\$ 550$ per door. To compensate for addition of shielding and installation of doors, the cost of a shielded container was estimated to be $\$ 50,000$, based on experience and engineering judgment.

During an inspection of potential deployment sites in the H-Area Tank Farm, a site east of Building 241-96H and adjacent to the Tanks 48-51 was identified as having sufficient area to accommodate up to three containers installed at ground level. 
Advantages of the skid-mounted deployment concept include maximization of off-site system fabrication, flexibility of location, and avoidance of potentially costly and time-consuming renovation of existing facilities. The primary disadvantage is the need to prepare a new site for system installation. However, site preparation of the containerized system is expected to be minimal.

\subsubsection{Selection of Deployment Concept}

Based on the information obtained and on assessment of the options available, deployment of the proposed CSSX-A system as a skid-mounted containerized system was selected as the preferred approach on which the project cost estimate has been based. Installation in Building 241-96H remains a viable option in the event that information obtained or developed during detailed design indicates that containerized deployment is unacceptable.

\subsection{SYSTEM MAINTENANCE CONCEPT}

To simplify system installation and operation, minimize cost, and expedite deployment, a direct maintenance approach is proposed for the CSSX-A system. Because of the presence of radionuclides within the process, direct maintenance can be performed only if the design includes provision for transfer of radionuclide-containing

process solutions from affected equipment into a separate shielded area and for rinsing of equipment to minimize dose to workers performing equipment replacement. It is envisioned that failed equipment located within shielded processing areas will be removed and replaced. No repairs will be performed within shielded enclosures unless the problem diagnosis is certain and the time required to implement repairs is less than the time required for equipment replacement.

\subsection{EQUIPMENT LAYOUT}

Based on selection of the skid-mounted containerized deployment concept, two equipment configurations were developed. The first layout locates the main processing line, including shielded process tanks, in a single shielded enclosure. This enclosure is installed as a second level above a shielded enclosure containing rework tanks, which 
receive system inventory and rinse solution to facilitate access to the main process line for maintenance. A third enclosure, which is not shielded, is installed at ground level adjacent to the shielded enclosures and contains cold chemical makeup tanks and transfer pumps. The cold chemical enclosure also includes an area for installation of a data archival and system monitoring station. Figure 7 shows plan and elevation views of equipment in the main shielded enclosure in accordance with the original configuration concept. The cold chemical makeup layout is presented in Fig. 8. The equipment arrangements shown confirm the feasibility of the deployment approach in terms of space requirements in that all equipment can be accommodated within the spatial confines of standard commercially available shipping containers.

The chief disadvantage of the first layout concept developed pertains to system maintenance. Since the system components most likely to fail (other than instrumentation) are the centrifugal contactors and pumps, the ability is needed to drain (via gravity) these equipment items and all other components that could contain radionuclides. If the radionuclide-containing tanks are located in the same enclosure as the pumps and contactors at roughly the same elevation, additional tankage is required at a lower elevation to receive all radionuclide-containing solutions from the main processing line.

To address these disadvantages, a second layout concept has been developed in which the contactors, pumps, and ancillary equipment (most notably, instrumentation) are located in a shielded enclosure mounted atop a separate shielded container that houses the radionuclide-containing aqueous solutions. To minimize radiolytic degradation of the solvent, the solvent inventory is located in the enclosure housing the contactor cascade.

The alternative layouts are shown in Figs. 9 and 10. Equipment configuration in the cold chemical makeup area is unchanged from that shown in Fig. 8.

\subsection{IMPACTS ON HLW SYSTEM}

The proposed process is fundamentally identical to the SWPF CSSX system. Consequently, the CSSX-A system will not pose any unique impacts on the SRS HLW system with regard to stream compositions. The primary composition-related consideration for integrating CSSX-A with the HLW system is the need to neutralize the 

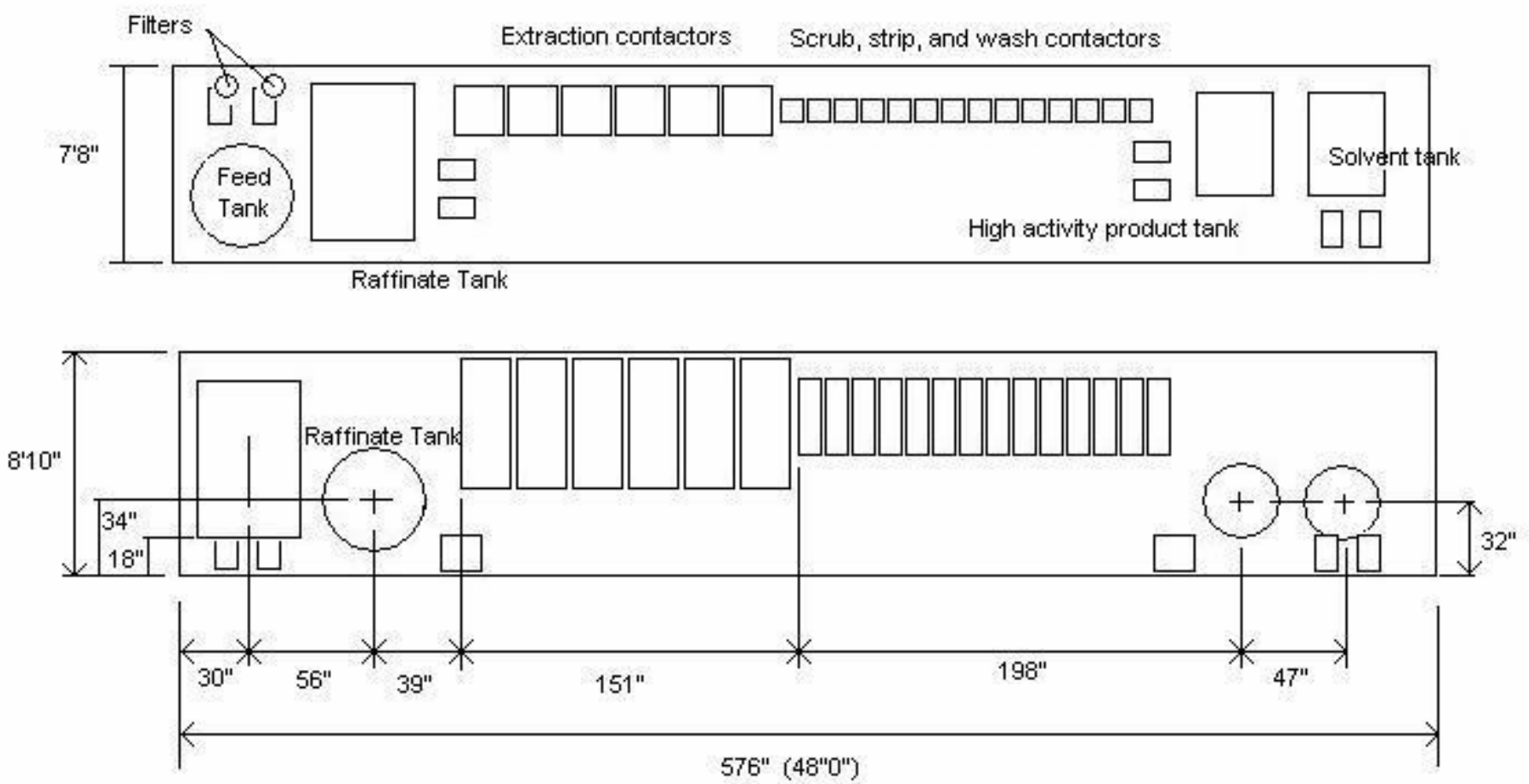

Fig. 7. Mainline process equipment arrangement, based on original system configuration concept. 

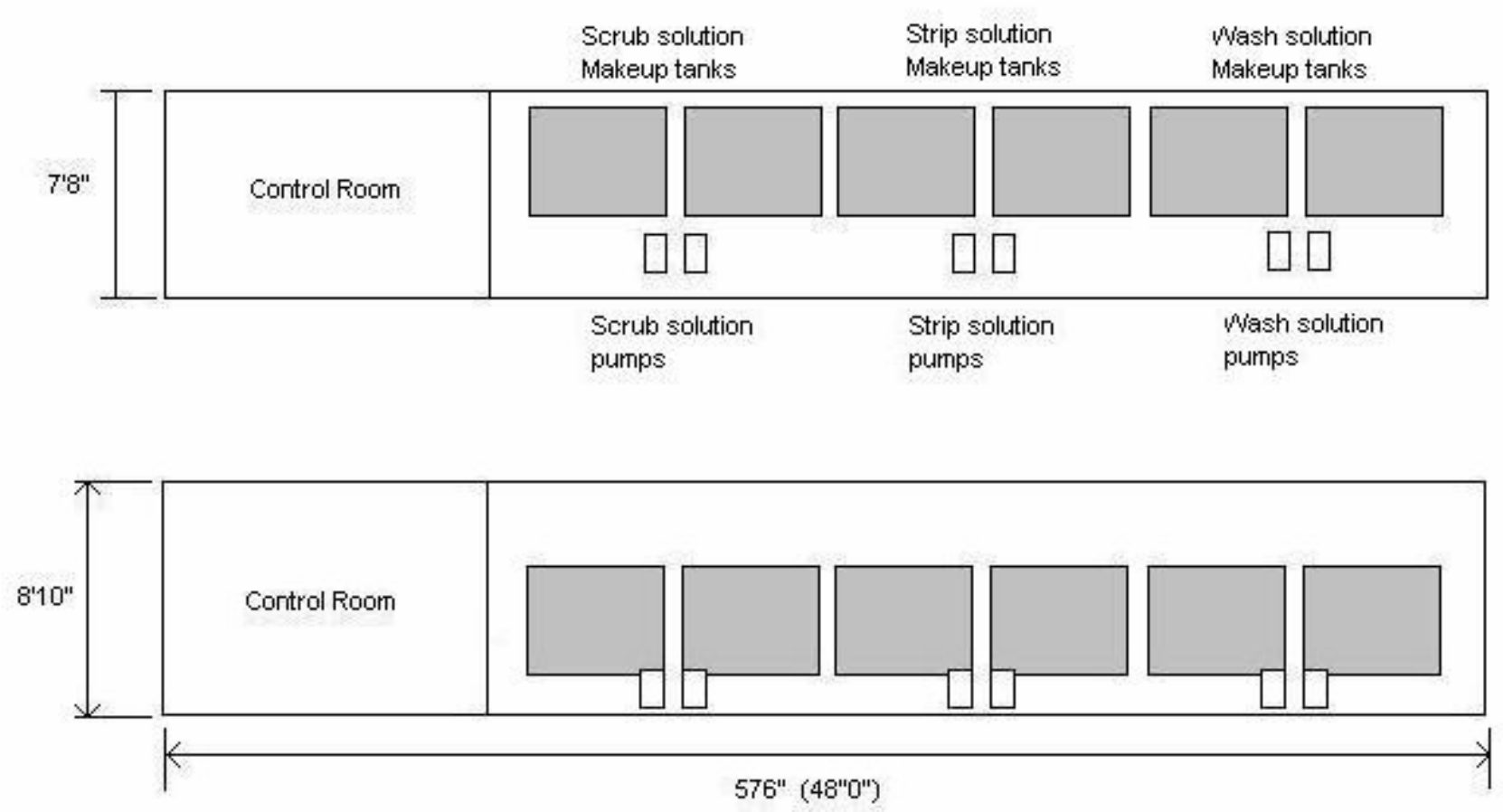

Fig. 8. Cold chemical makeup area equipment arrangement. 


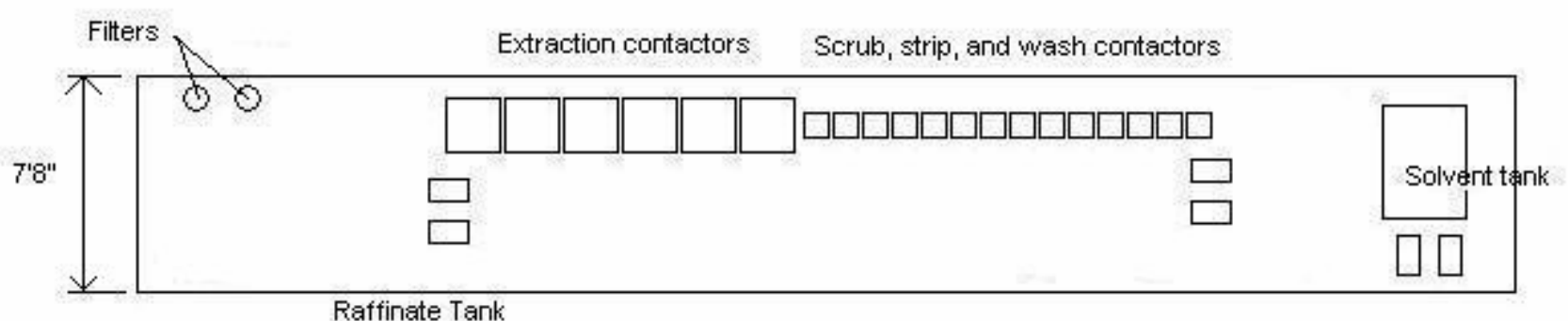

$\infty$

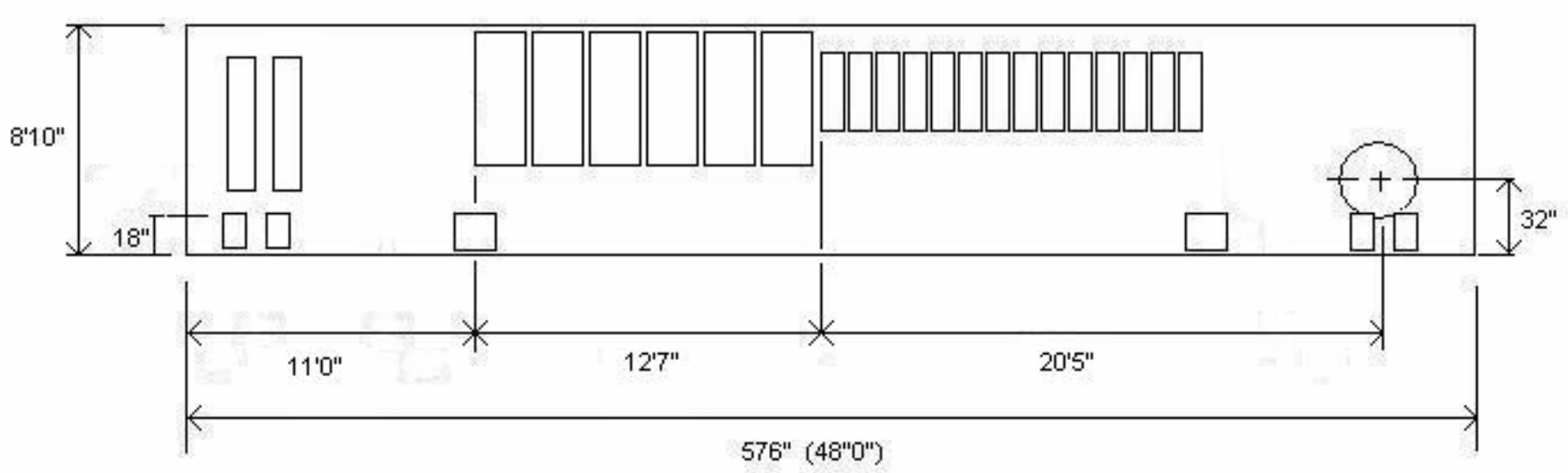

Fig. 9. Mainline process equipment arrangement, based on alternative configuration concept. 

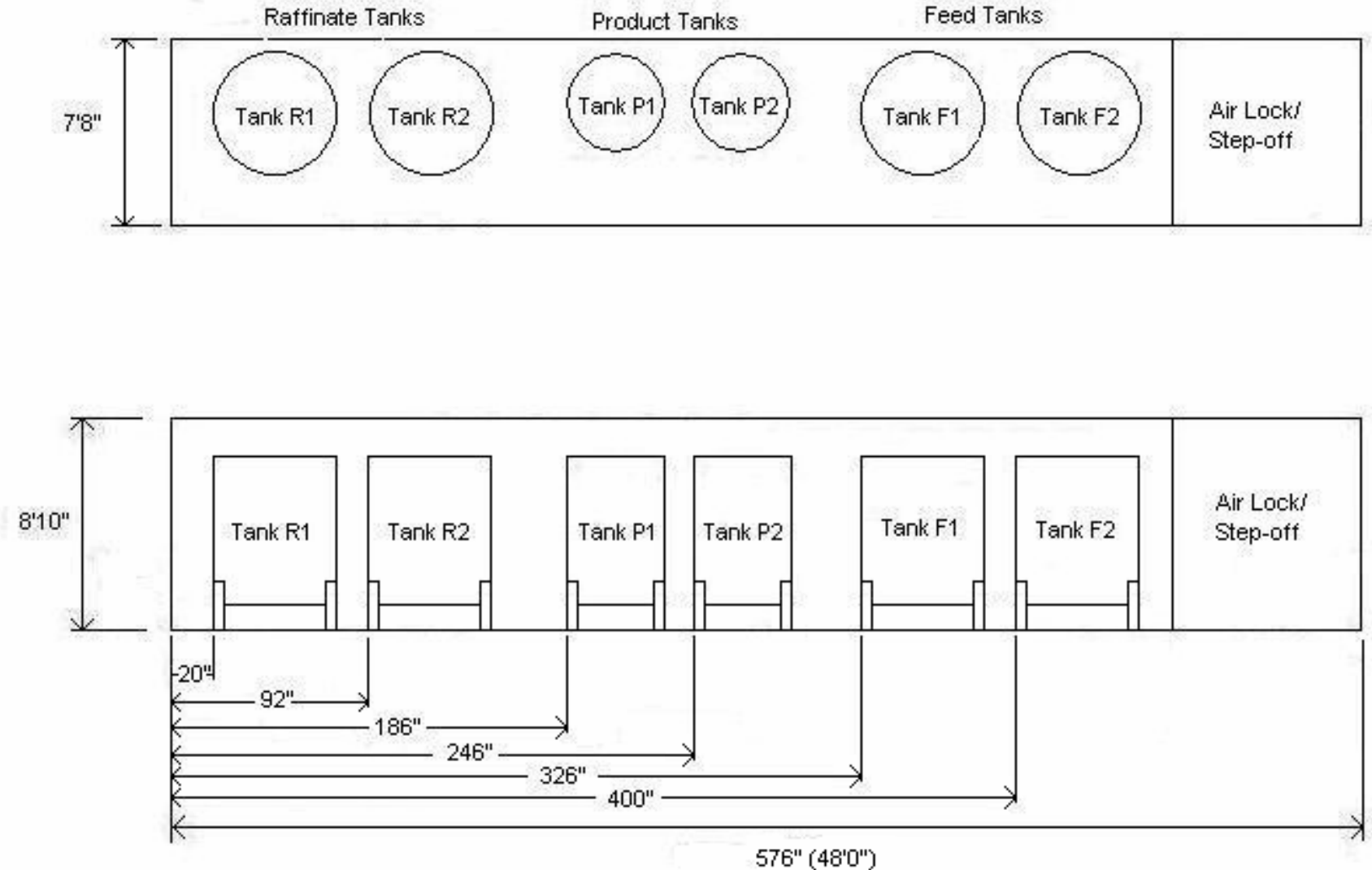

Fig. 10. Arrangement of aqueous feed and receiving tanks in alternative system configuration. 
acidic product solution prior to transfer to the DWPF feed tank (Tank 40) to prevent the possible precipitation of dissolved salt waste. The required $\mathrm{pH}$ adjustment has been accommodated in the CSSX-A system design. Consequently, the impacts of CSSX-A on the HLW system pertain to flow rates rather than composition.

Transfers to the HLW system were determined in the process of solving the CSSX-A process material balance and are presented in Table 6. As stated previously, it is anticipated that up to 100 gal of used solvent will require disposal annually. Disposal

alternatives for this waste stream are absorption onto oil dry followed by disposal as solid waste or transfer to the SWPF CSSX system for consolidation with used solvent from that facility.

\subsection{PROCESS CONTROL DESCRIPTION}

The process control scheme is based on the use of remotely monitored instrumentation and remotely actuated motors and valves. The process control diagram for the mainline process is presented in Fig. 11.

All valves, pumps, and contactor motors are remotely actuated. The commercial contactors on which the current design is based have variable speed capability. Variable

Table 6. Summary of transfer to the SRS HLW System from CSSX-A

\begin{tabular}{|c|c|c|c|c|}
\hline Source & Stream description & $\begin{array}{l}\text { Flow rate } \\
\text { gal } / \mathrm{min}\end{array}$ & $\begin{array}{c}\text { Annual flow } \\
\text { gal } \times 10^{6}\end{array}$ & Disposition \\
\hline $\begin{array}{l}\text { Solvent washing } \\
\text { contactor }\end{array}$ & $\begin{array}{l}\text { Aqueous wash sol'n } \\
\text { (dilute } \mathrm{NaOH} \text { ) }\end{array}$ & 0.42 & 0.2 & SMDF \\
\hline $\begin{array}{l}\text { Extraction system } \\
\text { raffinate }\end{array}$ & $\begin{array}{l}\text { Treated waste (aqueous } \\
\text { alkaline) }\end{array}$ & 6.76 & 3.2 & SMDF \\
\hline $\begin{array}{l}\text { Extraction system } \\
\text { product }\end{array}$ & $\begin{array}{l}\text { Concentrated Cs stream } \\
\left(\text { dilute } \mathrm{HNO}_{3}\right)\end{array}$ & 0.42 & 0.2 & DWPF \\
\hline Filter system & $\begin{array}{l}\text { Filter rinsate } \\
\text { (water/sludge) }\end{array}$ & 0.13 & 0.06 & DWPF \\
\hline
\end{tabular}

\footnotetext{
${ }^{a}$ Total flow to SMDF (Saltstone) $=3.4$ million gallons/year; total flow to DWPF
} $($ vitrification $)=0.26$ gallons $/$ year 


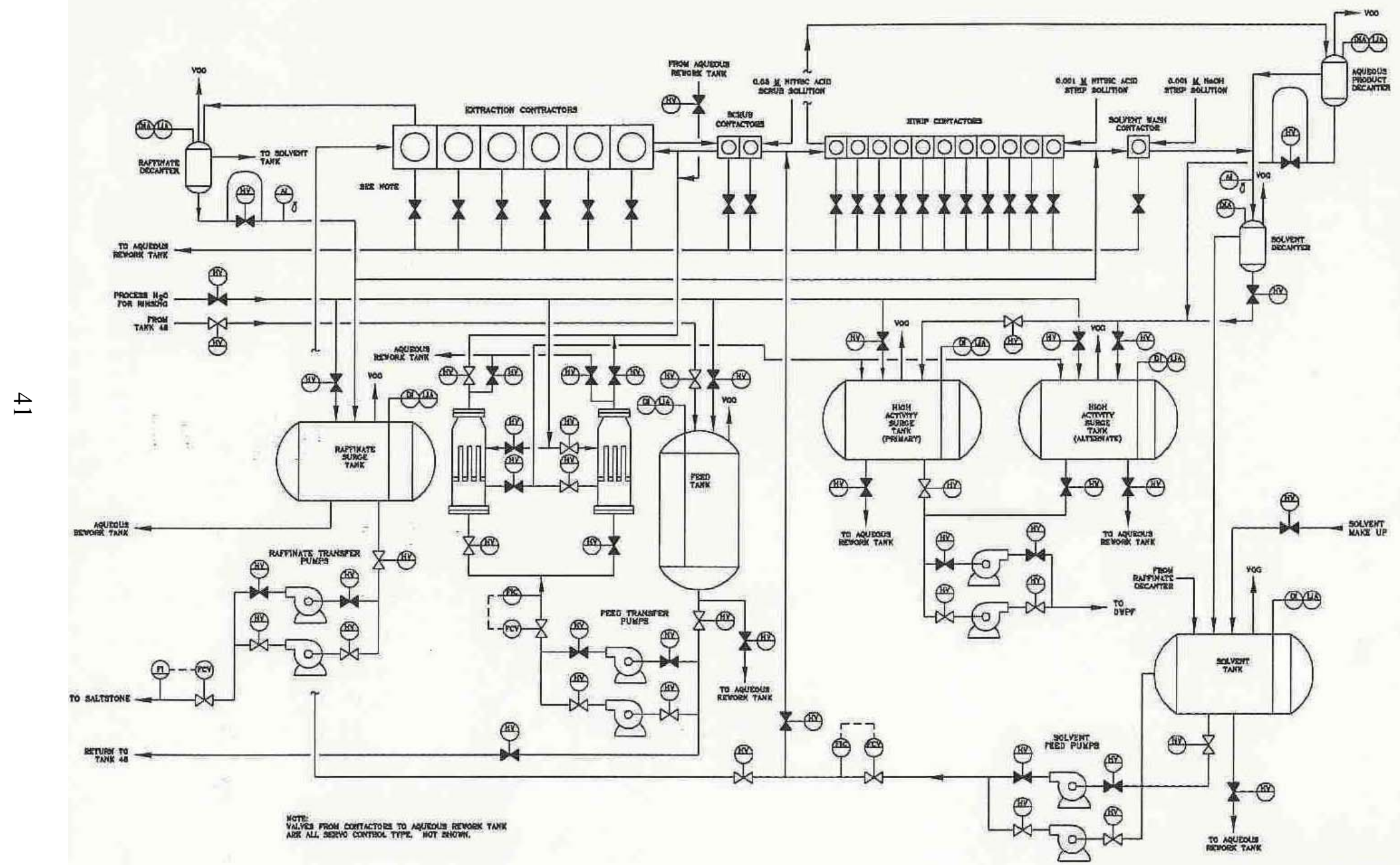

Fig. 11. CSSX-A system process instrumentation diagram. 
speed capability adds flexibility to the process and is useful in purging the system of the solvent phase without draining the system. It is recommended that this capability be retained in the system but that speed control for contactors in a specific section of the cascade be combined (e.g., all extraction contactors will be operated from one motor speed controller). Based on previous contactor experience, single contactor failures occur catastrophically; impending motor failure is indicated by increased current as well as by a drop in contactor speed. It is typically easier to reliably monitor current than it is to monitor rotor speed. Therefore, the individual contactor motor currents should be monitored and indicated.

Differential pressure indicators are installed across both filter housings to provide indication of element loading prior to filter plugging. Standard instrumentation on all tanks including decanters consists of level and density probes. In-line gamma monitors are to be installed in the raffinate and solvent effluent lines downstream of the applicable decanters to detect loss of extraction performance (raffinate stream indicator) or poor solvent stripping (stripped solvent indicator).

Flow indication from each feed line is key to process operation. Experience with contactor cascades indicates that as long as feed stream compositions remain within normal operating bounds, and as long as there are no upsets in contactor operation or flow rates, contactor system performance will remain within target parameters. Each feed to the system is indicated and monitored remotely. Deviations from setpoints are to be alarmed to the data acquisition system. The potential for producing large quantities of off-specification effluents in the event that a flow upset occurs dictates that the system be monitored constantly by a system operator.

\subsection{RISK ASSESSMENT}

As part of the design process, a list of potential risks to the successful deployment and operation of the CSSX-A system were identified. These risks can be divided into three categories: environmental, safety, and health (ES\&H) issues; factors 
affecting system operability and reliability; and technical uncertainties. Due to the highly developed state of CSSX technology, there are few technical issues, of which none pose the potential to make the technology unviable. Recommended development tasks needed to mitigate technical uncertainties are presented in Sect. 6 of this report.

ES\&H issues identified are typical of chemical processes involving radionuclides and include handling of hazardous materials, exposure to radiation, nuclear criticality, exposure to and release of gaseous emissions, accidental release of process solutions, use of electrical equipment, use of rotating machinery, and use of flammable chemicals. No heat sources are present in the CSSX-A system; hence, there are no thermal hazards. Operational risks, other than those posed by ES\&H issues and technical uncertainties, are posed primarily by the reliability of system components.

\subsubsection{Handling of Hazardous Materials}

Under normal operating conditions, the only direct handling of hazardous materials is the periodic formulation of aqueous scrub, strip, and solvent washing solutions. These operations will be performed in the unshielded cold chemical makeup area approximately once every $12 \mathrm{~h}$ for each of the three solutions. The solutions to be prepared are relatively dilute: $0.05 \mathrm{MHNO}_{3}, 0.001 M \mathrm{HNO}_{3}$, and $0.001 \mathrm{NaOH}$, respectively, for the scrub, strip, and wash solutions. However, formulation of each solution may involve dilution of fairly concentrated acid or caustic. Hazards associated with handling of these chemicals will be mitigated by use of appropriate personal protection equipment (PPE), including gloves, goggles and face shields, and protective clothing. Worker exposure to vapors during chemical formation will be mitigated by maintaining airflow through the enclosure. If deemed necessary as a result of a system safety analysis, a half-face or full-face respirator will be worn during formulation of cold chemical solutions.

Off-normal operations include removal and replacement of chemical equipment, miscellaneous hardware (e.g., valves), and failed instruments. To minimize exposure to hazardous chemicals, these operations will be performed only after solutions have been drained from the affected equipment. Where necessary due to the presence of radioactive components or concentrated hazardous chemicals, the affected component 
and associated process piping will be flushed with water prior to opening the system. PPE appropriate for the specific task will be utilized, in accordance with applicable safety requirements.

\subsubsection{Radiation Protection}

Radiation exposure of CSSX-A system operators and support personnel will be limited by installation of shielding around all areas of the process that contain radionuclides. Shielding calculations were performed for the system based on the original equipment configuration in which all mainline process components containing radiochemical solutions (tanks, contactors, and pumps) are located in a single enclosure. Calculations were performed for two cases: (1) the system containing the ${ }^{137} \mathrm{Cs}$ inventory present under normal operating conditions and (2) a worst-case scenario in which the extraction section of the system (contactors and tanks) is flooded with untreated feed and the product end of the system is flooded with concentrated product.

Under the base-case scenario, $8.1 \mathrm{~cm}$ (3.2 in.) of lead shielding is sufficient to lower the dose rate immediately outside the shielding to $2.5 \mathrm{mrem} / \mathrm{h}$. A summary of the shielding calculation results is presented in Appendix A.

Based on the original equipment layout, worker exposure during enclosure entry for maintenance or inspection will be minimized by draining the contents of the entire main processing line into tankage inside a separate shielded enclosure installed below the main process enclosure. In addition to draining the main process line, the equipment and interconnecting piping will be flushed of residual radionuclides.

The original equipment layout does not take advantage of the fact that failure of process tankage is a very low probability event, assuming proper quality inspection and assurance procedures are followed during fabrication. A second main process layout takes into account the reliability of tanks relative to moving components (pumps and contactors) by locating these components in a shielded enclosure separate from the tanks. Since the bulk of the radionuclide inventory is present in tankage, the dose in the main process line (i.e., the contactor cascade) is significantly reduced. In addition, preparation of the system for direct maintenance of contactors or pumps consists of draining and rinsing these process equipment items. 


\subsubsection{Criticality}

Based on waste characterization data and the source of the majority of the salt cake waste being processed (actinide recovery operations), the fissile material content of the material that will be treated in the CSSX-A system is too low to present the potential for a criticality event. Furthermore, the operations performed in the CSSX-A system cannot result in concentration of the system feed stream to such an extent that a criticality excursion can occur.

\subsubsection{Release of Gaseous Emissions}

Other than evaporation of process solutions, CSSX-A operations do not result in the generation of gaseous emissions.

The CSSX-A conceptual design includes installation of a dedicated ventilation system including a HEPA filtration system. All enclosures will be operated under negative pressure to prevent leakage of system atmosphere to the environment. All equipment will be sealed to the atmosphere. The only standard procedure requiring direct access to the system is the preparation of scrub, strip, and solvent washing solutions. These operations will be performed in the cold chemical makeup area. Respirators will be worn during chemical preparation procedures if needed, based on the results of a system safety analysis to be performed in conjunction with detailed design of the system.

\subsubsection{Accidental Release of Process Solutions}

To prevent release of process solutions to the environment, catch pans will be installed under equipment components. Each fluid mover will be mounted inside an individual catch pan equipped with duplicate level switches to indicate leakage. Similarly, each tank will be installed over a catch pan equipped with duplicate level switches. Each section of the CSSX-A contactor cascade, extraction, scrubbing, stripping, and solvent washing will be equipped with a catch pan with level sensing. Use of separate catch pans for each cascade section will aid in determining the source of any leak and will simplify dispositioning of any solutions collected. 
The process enclosures (containers) will serve as secondary leak containment. The interior of each enclosure that houses equipment containing radioactive solutions will be lined with stainless steel. The interior of the cold chemical makeup enclosure will be lined with stainless steel from the floor to a height sufficient to contain the complete solution inventory that is present when all chemical makeup tanks are full.

\subsubsection{Electrical Equipment}

Other than low-current instrument signals, electrical equipment within the system consists of pumps and centrifugal contactor motors. Both pumps and contactor motors are completely enclosed; there will be no open access to electrical circuits. Additional equipment will be installed as part of the ventilation, heating, and air condition system, which will be procured as a unit. All wiring and electrical connections will be installed according to applicable standards. The maximum voltage used in the CSSX-A system will be $220 \mathrm{~V}$.

\subsubsection{Use of Rotating Machinery}

Contactors and pumps (depending on type) contain rotating components. The specific contactors on which the current cost estimate is based may operate at speeds up to $5000 \mathrm{rpm}$. All rotating equipment used in the CSSX-A system is located inside housings to prevent direct access by system operators. In addition, dynamic balancing of each centrifugal contactor rotor will be performed by the vendor prior to its acceptance by the project.

\subsubsection{Fire Hazard}

Components of the solvent used in the CSSX-A process are flammable. Specifically, the flash point of trioctylamine is $163^{\circ} \mathrm{C}$ and the flash point of Isopar ${ }^{\circ} \mathrm{L}$ is $60^{\circ} \mathrm{C}$. There are no heat sources within the proposed system that could generate temperatures in excess of the flash point of any of the solvent components. A dedicated HVAC system has been incorporated into the design to maintain a temperature of approximately $25^{\circ} \mathrm{C}$ within the process enclosures, well below the flash point of all 
solvent components. As a further precaution against fire, the system design specifies use of totally enclosed explosion-proof motors for pumps and centrifugal contactors.

The cost estimate for the current design includes installation of a fire suppression system. Installation of this system was deemed necessary based on deployment of the system as a stand-alone containerized system, which precludes use of any existing suppression systems.

\subsubsection{Operational Risks}

Other than technical uncertainties that could impact system operation, the primary risk to operability is system reliability. The maintenance concept developed to address system failures has been described previously. The concept is based in part on the predicted reliability of contactor system components, which has been analyzed in the context of actinide recovery operation. The unavailability of the CSSX-A process due to failure of a contactor motor is projected to be 0.00403 (i.e., the system is unavailable $0.403 \%$ of the time due to motor malfunction). The unavailability of the system due to contactor bearing failure is estimated to be 0.0028 , while that due to pump failure is 0.00504 . The availability of the process based on availability of these particular components alone is 0.988 , or $98.8 \%$. By adding redundancy to the system via installation of duplicate pumps and excess stages to the process and by designing flexibility into the cascade through flow ratio variation, the availability of the process is predicted to exceed $99 \%$.

\subsection{TECHNICAL UNCERTAINTIES}

Due to the extensive effort that was undertaken to develop and demonstrate the CSSX process as an alternative to the In-Tank Precipitation Process, no experimental program was conducted in conjunction with the reported design effort. However, a small number of unresolved technical issues pertaining to CSSX have been identified and means to resolve them are proposed in this report. 


\subsubsection{Processing of Concentrated Feed}

Two issues identified during the subject effort result from the desire to increase the concentration of the waste feed stream to SWPF CSSX and the proposed CSSX-A system in order to increase the effective salt waste processing rate. Previous CSSX development tasks focused on treatment of waste containing sodium at 5.6 M. The current goal is to process waste that has been concentrated to a sodium level of $6.44 \mathrm{M}$. Concentration of the waste stream may affect cesium distribution under extraction conditions, since increased levels of sodium and potassium in the waste stream will lead to increased co-extraction of $\mathrm{Na}^{+}$and $\mathrm{K}^{+}$, potentially interfering with $\mathrm{Cs}^{+}$extraction.

To address these concerns, a thermodynamic model was used to predict distribution ratios in the extraction, scrubbing, and stripping sections of the cascade for the concentrated-feed case. ${ }^{6}$ In addition, the effect of combining the scrub and feed streams in the extraction section of the cascade has been taken into account in the modeling effort. The predicted extraction-condition values for the concentrated-feed case are lower than those obtained for less-concentrated feed without the contribution of the aqueous effluent from scrubbing. Consequently, the number of stages of extraction required for the concentrated case is greater than would have been determined using the previous $D_{C s}$ values for extraction.

\subsubsection{Need for Solvent Loss Mitigation}

Loss of solvent due to entrainment in the aqueous effluent streams from centrifugal contactors was observed in some tests performed during development and demonstration of the CSSX process. ${ }^{7}$ Results from other tests did not indicate the presence of entrained solvent in these effluents. ${ }^{8}$ The ability to remove entrained organic has been demonstrated using both decanters and coalescers. Previous results indicate that use of decanters is impractical for large-scale CSSX-type operations; the size of the decanters required to obtain sufficient residence time for effective separation has a severe impact on the process footprint. Results from coalescer tests indicate that up to $99 \%$ removal of entrained solvent from aqueous solutions is achievable.

Prior indications of entrained solvent were observed in tests using laboratoryand pilot-scale centrifugal contactors. Contactors used in the CSSX-A system will be 
significantly larger and will generate considerably higher centrifugal forces in the separating zones. Consequently, phase-separation performance is likely to be improved in the larger units. The need for addition of coalescers to the CSSX-A flowsheet will be determined based on results of phase-separation tests using full-scale contactors, which will be performed prior to final design of the system.

\subsubsection{Contactor Hydraulic and Mass Transfer Performance}

Extensive contactor testing was performed during the CSSX technology development and demonstration effort, using both simulated and real salt waste. All tests performed thus far have utilized laboratory-scale (2-cm-diam rotor) or bench-scale (5- and 5.5-cm-diam rotor) contactors. Acceptable mass transfer and phase-separation results have been obtained in previous tests using these small contactors. It is anticipated that due to the larger amount of energy input to the liquid in the mixing zone of larger contactors and as a result of the higher centrifugal forces generated using larger rotor diameters, both mass transfer and phase-separation performance will be improved over previous results and will exceed the performance metrics on which the current system design is based. Nonetheless, the performance of contactors of the sizes needed in the various sections of the CSSX-A cascade needs to be verified and quantified to increase confidence in the CSSX-A system design.

\subsubsection{In-line Gamma Monitoring}

Commercial in-line gamma monitoring is available. However, in-line gamma monitoring has not been fully demonstrated in applications at SRS. Therefore, demonstration of this capability in a test mock-up or in conjunction with other waste treatment technology demonstrations may be required to achieve acceptance of the technology prior to its deployment in the CSSX-A system. 


\section{ECONOMIC ANALYSIS}

Many benefits derived by deployment of the proposed technology cannot be described in quantitative terms. This limitation is due in part to the nature of the specific benefits obtained. For example, it is not possible to place a value on the benefit to SWPF CSSX deployment resulting from the design, fabrication, installation, and operating information obtained from the CSSX-A system. In other instances, the scope of the Phase 1 activity and the accelerated schedule imposed on the effort precluded the performance of in-depth economic benefit assessments.

Qualitative benefits that will be realized by deploying the CSSX-A processing alternative include:

- verification of full-scale contactor operation in support of SWPF CSSX design and deployment;

- development of design, fabrication, installation, and operation experience bases that will be at least partially applicable to the SWPF CSSX system; and

- availability of the CSSX-A system as a means of providing contactor-based solvent extraction system operating experience to SRS HLW operations personnel.

\subsection{BASIS FOR COMPARISON WITH BASELINE}

As discussed previously, the original plan for treatment of SRS HLW was to treat all stored supernates and dissolved salt cake through the SWPF. Initially, processing would be performed in a reduced-scale facility that would serve to demonstrate the SWPF component systems (MST actinide removal, filtration, and CSSX). A full-scale SWPF system operating at an annual throughput of 6 million gallons would incorporate lessons learned from the demonstration flowsheet and would come on-line 2 to 3 years after the reduced-scale facility. On the assumption that the demonstration-scale facility would operate at an annual throughput of 1 million gallons 
and would begin operation at the beginning of FY 2007 and that the full-scale SWPF would come on-line at the beginning of FY 2010, processing of all HLW supernates and dissolved salt cake would require 12 to 14 years. The lower time-to-process is achieved if the demonstration facility is operated in parallel with the full-scale SWPF for the duration of remediation.

To accelerate closure of the SRS HLW tank farms, DOE and the Westinghouse Savannah River Company have proposed to treat waste according to its actinide, strontium, and cesium content. Using this approach, only supernates, interstitial liquid, and the fraction of dissolved salt cake containing high levels of actinides and cesium will be treated in the SWPF. Low-curie salt will be immobilized as saltstone with no treatment following dissolution and retrieval. Dissolved salt cake containing actinides and/or strontium at levels exceeding Saltstone WAC but containing only low levels of cesium will be treated in an Actinide Removal Process, with the treated material being sent to the Saltstone facility for immobilization. Material that contains high levels of actinides and cesium will be transferred to the SWPF for cesium removal after processing in the Actinide Removal Process. Salt cake waste that exceeds the Saltstone WAC due to cesium concentration only will be processed in the SWPF. It is anticipated that approximately one-third of the combined supernate and dissolved salt cake inventory will require treatment in the SWPF, one-third can be characterized as lowcurie salt, and one-third will require treatment in the Actinide Removal Process.

It is highly probable that the installation and operation of a demonstration-scale facility will be eliminated in the accelerated-closure approach. Most likely, a reducedscale SWPF will be constructed, possibly having one-half the throughput of the original full-scale plant concept. Unfortunately, cost data for a reduced-scale SWPF are not available for comparison in the current cost/benefit analysis. Therefore, the cost/benefit analysis performed for the proposed CSSX-A process includes a primary comparison with the original SWPF deployment plan in which the plant capacity is 6 million gallons/year. This comparison assumes that a full-scale SWPF is constructed and operated and that the cost savings from CSSX-A deployment result from early tank farm closure and reduced SWPF operating costs due to diversion of material to the CSSX-A system. A second comparison has been made with the reduced-scale SWPF deployment 
(projected at $50 \%$ of the original plan), using the assumptions that the operating costs are unchanged from the full-scale SWPF and that facility costs can be scaled against throughput using a constant factor.

To reflect uncertainties regarding the level of cesium that will be allowed in waste that can be immobilized as saltstone, analyses have been performed on the bases of 74 million gallons and 37 million gallons of dissolved salt cake treated by cesium removal. In all cases, it has been assumed that 18 million gallons of dissolved salt cake requires a degree of cesium removal that can be accomplished using the proposed alternative process (CSSX-A).

\subsection{FIXED CAPITAL INVESTMENT ESTIMATE}

The estimate of fixed capital investment begins with selection and costing of the major purchased but uninstalled major equipment items. The estimate of fixed capital investment includes the direct and indirect costs associated with installing the major equipment items into an operable facility. The fire suppression equipment is provided as a system with little installation cost, so its cost is added to the investment estimate without the imposition of additional direct and indirect costs.

The costs of purchased major equipment items, presented in Table 7, are valid for mid-year 2003. The contactor cascade cost estimate is based on a previous ORNL procurement of a similar contactor cascade; current costs have been scaled from $\$ 37,500$ per stage for 8.4-cm contactors procured in 1992. Tank costs were calculated based on size and material of construction using a standard estimating procedure. ${ }^{9}$ Other costs were budget quotes from the sources referenced. Costs for shipping major equipment items to the facility site were assumed to be zero.

The primary construction material for all equipment surfaces contacting process solutions is 300 series stainless steel, with selection of appropriate grade reserved for the detailed design phase of this project. The design and selection of equipment here are based primarily on feasibility and cost considerations; these selections will be reviewed during detailed design. 
Table 7. Purchased costs of major equipment items

\begin{tabular}{lr}
\hline \multicolumn{1}{c}{ Equipment } & \multicolumn{1}{c}{ Cost } \\
Cascade & $\$ 1,034,904^{a}$ \\
Tankage & $\$ 346,846$ (ref. 9) \\
Filters & $\$ 72,000$ (ref. 10) \\
Pumps & $\$ 110,000$ (ref. 11) \\
Equipment containers & $\$ 70,000$ (ref. 15) \\
Air filtration system & $\$ 50,000$ (ref. 12) \\
Fire suppression & $\$ 49,119$ (ref. 13) \\
Total purchased equipment & $\$ 1,732,869$ \\
\hline${ }^{a}$ Engineering estimate
\end{tabular}

${ }^{a}$ Engineering estimate.

The cascade utilizes appropriately sized centrifugal contactors of a standard design by CTI of Cedar Park, Texas. These units have interchangeable heavy-phase weir plates to facilitate optimization of phase-separation performance for specific twophase systems. Determination of the optimum heavy-phase (aqueous) weir diameter has been identified as a key prerequisite to detailed design of the CSSX-A system.

The tankage for the process is designed for less than 50-psi service; this pressure rating is subject to review during detailed design. The backwashable feed filtration system is of a standard design from Mott Corp. of Farmington, Connecticut and is composed of two filter vessels, each containing several porous metal filter elements of approximately 2.5-in. diameter and 60-in. length. ${ }^{10}$ The filters are expected to retain approximately $90 \%$ of particles larger that $1 \mu \mathrm{m}$. The pumps included in the current design and cost estimate are of canned rotor leakproof design from Chempump of Warrington, Pennsylvania. ${ }^{11}$

Three containers for housing the equipment have exterior dimensions of $48-\mathrm{ft}$ (length) by 8 -ft (height) by 8.5 -ft (width). The container exterior is painted mild steel with additional support structure added to a commercial design so that the equivalent of 3 -in. thickness of lead shielding may be added on the side and top surfaces; the interior of the containers is lined with stainless steel sheet with the floor installed so that any leaks will be contained and routed through an appropriate collection system. The 
containers are provided with doorways (with an air lock) so that maintenance personnel may enter and leave.

The air handling and filtration system is designed to maintain a differential pressure of 2-in. of water with a maximum airflow rate of 15,000 scfm. The air handling and filtration system utilizes HEPA filters with bagout housings and expected efficiency of $99.97 \% .^{12}$ The halon-based fire suppression system is of standard design by International Equipment ${ }^{13}$ and utilizes units for each equipment module; the cost shown Table 7 is for a fully installed unit.

The fixed capital investment is presented in Table 8. It should be noted that this investment estimate does not contain startup funds or working capital. The purchased equipment costs in Table 7 include the cost of a fire suppression system, which is added without direct and indirect installation costs. The costs in Table 8 were estimated using multipliers taken from the U.S. chemical industry and modified for application to processing of radioactive materials. ${ }^{14}$ The total fixed capital cost shown in Table 8 includes the purchase of an initial charge of solvent to the system (approximately 100 gal at $\$ 5500 / \mathrm{gal})$.

\subsection{ESTIMATION OF ANNUAL OPERATING COSTS}

Operating costs for a single year of operation have been estimated based on current cost rates (FY 2003) without escalation and are presented in Table 9. The

estimating procedure is typical of that found in the U.S. chemical industry. ${ }^{14}$ The determination of the operating labor required per shift is based on discussions with SRS personnel in summer of 2003; the hourly rate for operating labor is $\$ 53.00$ with $35 \%$ overhead.

\subsection{LIFE-CYCLE COSTS AND COST/BENEFIT ANALYSIS}

Life-cycle costs are presented for the proposed CSSX-A facility with no consideration of inflation on the cost elements, which are valid for mid-2003. An upfront development cost (for resolution of identified technical uncertainties) has been added to the estimate of fixed capital, which was presented previously. The basic cost elements are presented in Table 10. 
Table 8. Estimate of fixed capital investment

\begin{tabular}{|c|c|c|}
\hline Cost element & Cost & $\begin{array}{c}\text { Percentage of purchased } \\
\text { equipment cost }\end{array}$ \\
\hline \multicolumn{3}{|l|}{ Direct expenses } \\
\hline Purchased equipment (major) & $\$ 1,683,750$ & 100 \\
\hline Installation & $\$ 791,363$ & 47 \\
\hline Instrumentation & $\$ 505,125$ & 30 \\
\hline Piping & $\$ 1,347,000$ & 80 \\
\hline Electrical & $\$ 336,750$ & 20 \\
\hline Buildings & $\$ 589,313$ & 35 \\
\hline Yard improvement & $\$ 0$ & 0 \\
\hline Service facilities & $\$ 0$ & 0 \\
\hline SUBTOTAL, Direct expenses & $\$ 5,253,301$ & 312 \\
\hline \multicolumn{3}{|l|}{ Indirect expenses } \\
\hline Engr. \& supervision & $\$ 555,638$ & 33 \\
\hline Construction expenses & $\$ 690,338$ & 41 \\
\hline Contractor fee & $\$ 353,588$ & 21 \\
\hline $\begin{array}{l}\text { Contingencies } \\
\text { (20\% Direct \& indirect) }\end{array}$ & $\$ 1,713,216$ & 102 \\
\hline TOTAL EXPENSES & $\$ 8,566,081$ & 509 \\
\hline $\begin{array}{l}\text { TOTAL EXPENSES } \\
\text { (with fire suppression) }\end{array}$ & $\$ 8,615,200$ & \\
\hline Purchase of solvent for startup & $\$ 550,000$ & \\
\hline $\begin{array}{l}\text { TOTAL FIXED CAPITAL } \\
\text { INVESTMENT } \\
\text { (including initial solvent) }\end{array}$ & $\$ 9,165,200$ & \\
\hline
\end{tabular}


Table 9. Annual direct operational cost estimate

\begin{tabular}{lrrr}
\hline \multicolumn{1}{c}{$\begin{array}{c}\text { Cost element } \\
\text { Raw materials }\end{array}$} & Cost basis & Quantity per year & Cost per year \\
$\mathrm{HNO}_{3}$ & $\$ 0.108 / \mathrm{lb}$ & $5952 \mathrm{lb}$ & $\$ 643$ \\
$\mathrm{NaOH}$ & $\$ 0.093 / \mathrm{lb}$ & $741 \mathrm{lb}$ & $\$ 69$ \\
Solvent & & & \\
$\quad$ Makeup & $\$ 5,500 / \mathrm{gal}$ & $100 \mathrm{gal}$ & $\$ 550,000$ \\
$\quad$ Replacement & $\$ 5,500 / \mathrm{gal}$ & 0 & $\$ 0$ \\
& & & \\
Operating labor & $\$ 72 / \mathrm{h}$ (incl. $35 \%$ ovhd) & $15,545 \mathrm{~h}$ & $\$ 1,119,240$ \\
$\begin{array}{l}\text { Supervision \& } \\
\text { clerical labor }\end{array}$ & $15 \%$ of operating labor & & $\$ 167,886$ \\
Utilities & & & \\
Electricity & $\$ 0.05 / \mathrm{kWh}$ & $545,571 \mathrm{kWh}$ & $\$ 27,279$ \\
Process water & $\$ 0.0019 / \mathrm{gal}$ & $532,484 \mathrm{gal}$ & $\$ 1,012$ \\
Cooling water & $\$ 0.0003 / \mathrm{gal}$ & $3,878,330 \mathrm{gal}$ & $\$ 1,164$ \\
Waste disposal & $\$ 13.82 / \mathrm{gal}$ & $100 \mathrm{gal}$ & $\$ 1,382$ \\
& & & $\$ 549,912$ \\
Maint. and repair & $6 \%$ of fixed capital & & $\$ 82,487$ \\
Operating supplies & $15 \%$ of maint. and repairs & & $\$ 166,837$ \\
Laboratory charges & $15 \%$ of operating labor & & $\$ 2,667,911$ \\
\hline Total & & & \\
\hline
\end{tabular}

Table 10. Life-cycle cost elements

\begin{tabular}{ll}
\hline Cost element & Cost value \\
Fixed capital investment & $\$ 9,165,200$ \\
& $\$ 2,734,801$ \\
Development costs & $\$ 2,667,911$ \\
\hline
\end{tabular}


The life-cycle cost varies as the project lifetime is extended and the total volume of waste treated increases. Life-cycle costs as a function of lifetime are presented in Table 11 without escalation, based on an assumed constant treatment rate of 3 million gallons of waste per year. The life-cycle costs are estimated as the sum of the total fixed capital investment, the development costs, and the product of the years of operation and the annual operating costs.

The life-cycle cost of the CSSX-A system operating over 6 years is approximately $\$ 27,400,000$. The cost/benefit analysis is presented in Table 12 for an SWPF CSSX system that processes a total of 74 million gallons of waste. The cost/benefit analysis results shown in Table 12 are based on the assumption that all waste requires cesium removal and that the benefit is derived by diverting a fraction of the waste stream to the CSSX-A process, thereby accelerating dispositioning. In the column headed "Accelerated Case A," the operating costs for the SWPF CSSX system are reduced proportionally to the fraction of waste that is processed in the CSSX-A system (18 million gallons); for this comparison, the benefit-to-cost ratio is estimated to be 20. In Case A it is assumed that the capacity of the SWPF CSSX system is not changed from the baseline in order to expedite HLW dispositioning, and that there is no reduction in SWPF capital costs. In the column headed "Accelerated Case B," the Case A reduction in operating costs was used and the total project cost of SWPF CSSX has been revised downward to reflect reduced SWPF CSSX throughput, by the 0.6 power of the throughput reduction (i.e., a factor of 2). The cost/benefit ratio for this case is estimated to be 47. By coincidence, the rate of processing in CSSX-A is half the rate in the baseline SWPF CSSX system. Therefore the scale reduction in SWPF CSSX due to CSSX-A operation results in a 50\%-of-baseline SWPF plant-the currently anticipated full-scale SWPF capacity.

The cost/benefit analysis presented in Table 13 is based on a need to treat 37 million gallons of dissolved salt cake. This basis assumes that the regulatory limit for ${ }^{137} \mathrm{Cs}$ in saltstone is increased to permit diversion of approximately half of the total waste inventory directly to the Saltstone processing facility without prior treatment in SWPF. The baseline operating costs were revised prior to the cost/benefit analysis 
Table 11. Life-cycle cost as a function of project lifetime

\begin{tabular}{cc}
\hline $\begin{array}{c}\text { Years of } \\
\text { operation }\end{array}$ & $\begin{array}{c}\text { Life-cycle cost } \\
\text { \$/gal }\end{array}$ \\
\hline 1 & 4.67 \\
2 & 2.78 \\
3 & 2.15 \\
4 & 1.84 \\
5 & 1.65 \\
6 & 1.52 \\
7 & 1.43 \\
8 & 1.36 \\
9 & 1.31 \\
10 & 1.27 \\
\hline
\end{tabular}

proportional to the quantity of materials processed. In the column headed "Accelerated Case A1," the operating costs of SWPF are reduced proportionately to the quantity of material diverted to CSSX-A (18 million gallons); for this comparison, the benefit-tocost ratio is estimated at 20. In the column headed "Accelerated Case B1," the previous revision of operating costs was used and the total project cost of CSSX was revised downward by the 0.6 power of the ratio of materials processed in CSSX; the benefit-tocost ratio for this case is estimated at 46.

It should be noted that the difference in benefits between Cases A and A1 and between Cases B and B1 are minimal; this reflects the relatively minor fraction of the life-cycle cost that is attributable to operating costs.

Significant uncertainties in the salt waste dispositioning program exist, any of which can invalidate the results of the cost/benefit analyses presented in Tables 12 and 13. These uncertainties include

1. the processing capacity of the SWPF

2. the level of ${ }^{137} \mathrm{Cs}$ that can be processed in the Saltstone facility due to shielding or other processing-related parameters, 
3. the level of ${ }^{137} \mathrm{Cs}$ that state and/or federal regulations permit to be immobilized in saltstone,

4. the efficacy of interstitial supernate removal and its impact on the ${ }^{137} \mathrm{Cs}$ concentrations in dissolved salt cake.

Table 12. Cost/benefit analysis for an SWPF CSSX baseline of 74 million gallons ${ }^{a}$

\begin{tabular}{lrrr}
\hline Life Cycle Costs for CSSX & Baseline (SWPF) & $\begin{array}{c}\text { Accelerated } \\
\text { Case A }^{\mathrm{b}}\end{array}$ & $\begin{array}{c}\text { Accelerated } \\
\text { Case B }^{c}\end{array}$ \\
Volume processed (gal) & $7.40 \mathrm{E}+07$ & $5.60 \mathrm{E}+07$ & $5.60 \mathrm{E}+07$ \\
Total project cost, $\$$ & $1.60 \mathrm{E}+09$ & $1.60 \mathrm{E}+09$ & $1.06 \mathrm{E}+09$ \\
Operating cost, $\$$ & $1.49 \mathrm{E}+09$ & $1.13 \mathrm{E}+09$ & $1.13 \mathrm{E}+09$ \\
Life-cycle costs, $\$$ & $3.09 \mathrm{E}+09$ & $2.73 \mathrm{E}+09$ & $2.19 \mathrm{E}+09$ \\
Cost per gallon $(\mathrm{CSSX}), \$$ & 42 & 49 & 39 \\
Tank closure savings, ${ }^{d} \$$ & Baseline & $2.04 \mathrm{E}+08$ & $4.08 \mathrm{E}+08$ \\
Benefit (savings), $\$$ & 0 & $5.64 \mathrm{E}+08$ & $1.31 \mathrm{E}+09$ \\
Life-cycle-cost $(\mathrm{CSSX}-\mathrm{A}), \$$ & & $2.74 \mathrm{E}+07$ & $2.74 \mathrm{E}+07$ \\
Net cost savings, $\$$ & & $5.37 \mathrm{E}+08$ & $1.28 \mathrm{E}+09$ \\
Benefit-to-cost ratio & & 20 & 47 \\
\hline
\end{tabular}

${ }^{a}$ The baseline quantity of material potentially processed in CSSX is assumed to be 74 million gallons. CSSX-A processes 18 million gallons at 3 million gallons per year.

${ }^{b}$ Accelerated Case A is based on operation of CSSX-A in conjunction with the full-scale SWPF. Total cost includes reduced operating costs relative to the stand-alone full-scale SWPF baseline, which are proportional to reduced number of gallons treated.

${ }^{c}$ Accelerated Case B is based on operation of CSSX-A in conjunction with a 50\%-scale SWPF. The total cost includes reductions in operating costs (similar to Case A) and the system capital cost, the latter using the six-tenths rule on the basis of reduced throughput.

${ }^{d}$ Tank Farm cost savings are estimated at $\$ 68$ million/year, based on early closure of Tank Farm F and consolidation of wastes in Tank Farm H as a result of CSSX-A operation. Increased tank closure rate of 3 million gallons/year will lead to closure in 18/3 (i.e., 6) fewer years compared with the stand-alone reduced-throughput SWPF. The increased tank closure rate of 6 million gallons/year will lead to closure in 18/6 (i.e., 3) fewer years compared with the baseline SWPF without CSSX-A. 
Table 13. Cost/benefit analysis for an SWPF CSSX baseline of 37 million gallons ${ }^{a}$

\begin{tabular}{lrrr}
\hline Life-cycle costs for CSSX & Baseline (SWPF) & $\begin{array}{r}\text { Accelerated } \\
\text { Case A1 }\end{array}$ & $\begin{array}{r}\text { Accelerated } \\
\text { Case B1 }\end{array}$ \\
Volume processed, gal & $3.70 \mathrm{E}+07$ & $1.90 \mathrm{E}+07$ & $1.90 \mathrm{E}+07$ \\
Total plant cost, $\$$ & $1.60 \mathrm{E}+09$ & $1.60 \mathrm{E}+09$ & $1.07 \mathrm{E}+09$ \\
Operating cost, $\$$ & $7.45 \mathrm{E}+08$ & $3.83 \mathrm{E}+08$ & $3.83 \mathrm{E}+08$ \\
Life-cycle cost, $\$$ & $2.35 \mathrm{E}+09$ & $1.98 \mathrm{E}+09$ & $1.46 \mathrm{E}+09$ \\
Cost per gallon $(\mathrm{CSSX}), \$$ & 63 & 104 & 77 \\
Tank closure savings, $\$$ & Baseline & $2.04 \mathrm{E}+08$ & $4.08 \mathrm{E}+08$ \\
Benefit (savings), $\$$ & 0 & $5.74 \mathrm{E}+08$ & $1.30 \mathrm{E}+09$ \\
Life-cycle cost CSSX-A, $\$$ & & $2.74 \mathrm{E}+07$ & $2.74 \mathrm{E}+07$ \\
Net cost savings, $\$$ & & $5.47 \mathrm{E}+08$ & $1.27 \mathrm{E}+09$ \\
Benefit-to-cost ratio & & 20 & 46 \\
\hline
\end{tabular}

${ }^{a}$ CSSX-A processes 18 million gallons at 3 million gallons per year.

${ }^{b}$ Accelerated Case A1 has reduced operating costs proportional to the reduced number of gallons treated.

${ }^{c}$ Accelerated Case B1 has reduced operating costs and reduced CSSX capital cost using the six-tenths rule.

${ }^{d}$ Tank Farm cost savings are estimated at $\$ 68$ million/year, based on early closure of Tank Farm F and consolidation of wastes in Tank Farm $\mathrm{H}$ as a result of CSSX-A operation. Increased tank closure rate of 3 million gallons/year will lead to closure in 18/3 (i.e., 6) fewer years compared with the stand-alone, reduced-throughput SWPF. Increased tank closure rate of 6 million gallons/year will lead to closure in 18/6 (i.e., 3) fewer years compared with the baseline SWPF without CSSX-A.

Items 2, 3, and 4 have potentially significant impacts on the both the SWPF processing capacity determination and on the volume of dissolved salt cake that can be treated in CSSX-A (or any other alternative treatment technology that has a lower decontamination capability than the SWPF CSSX system).

To obtain a benefit determination independent of these uncertainties, a simple determination of cost saved due to early closure of the HLW tank farms has been made. The results of this analysis are presented in Table 14. Two cases (C and D) have been considered. In Case $\mathrm{C}$, the total volume of waste that can be treated to meet the Saltstone facility WAC for ${ }^{137} \mathrm{Cs}$ using the CSSX-A system is limited by system 
Table 14. Cost savings based solely on accelerated tank farm closure.

\begin{tabular}{|c|c|c|c|c|c|}
\hline Case $C$ & $\begin{array}{c}\text { Waste treated } \\
\text { in SWPF } \\
\text { (gal) }\end{array}$ & $\begin{array}{c}\text { Waste treated } \\
\text { in CSSX-A } \\
\text { (gal) } \\
18 F+06\end{array}$ & $\begin{array}{c}\text { SWPF } \\
\text { start date/ } \\
\text { end date } \\
2010 / 2016\end{array}$ & $\begin{array}{l}\text { CSSX-A } \\
\text { start date/ } \\
\text { end date } \\
2006 / 2012\end{array}$ & $\begin{array}{c}\text { Savings } \\
\text { w/ CSSX-A } \\
(\$)^{a} \\
12 \mathrm{~F}+09\end{array}$ \\
\hline $\begin{array}{l}\text { Case C } \\
\text { w/o CSSX-A }\end{array}$ & $55 \mathrm{E}+06$ & 0 & $2010 / 2028$ & & \\
\hline Case D & $42 \mathrm{E}+06$ & $32 \mathrm{E}+06$ & $2010 / 2017$ & $2006 / 2017$ & $5 \mathrm{E}+08$ \\
\hline $\begin{array}{l}\text { Case D } \\
\text { w/o CSSX-A }\end{array}$ & $74 \mathrm{E}+06$ & 0 & $2010 / 2022$ & & \\
\hline
\end{tabular}

${ }^{a}$ Savings are based on early tank farm closure, and have been calculated by multiplying the number of years of reduced tank farm operation by an estimated annual tank farm operation cost of $\$ 100$ million.

capability to 18 million gallons, with an additional 37 million gallons of waste requiring treatment in SWPF. In Case D, 74 million gallons of waste requires cesium removal, with up to 37 million gallons being treated using CSSX-A. In the first case, the basis for comparison is use of a 50\% SWPF (3 million gallon/year capacity) to perform all cesium removal treatment, with SWPF startup in 2010. In the second case, the comparison is made with the full-scale (6 million gallon/year) plant. Different throughputs are established for the comparison cases because processing 74 million gallons of waste (Case D) in a 50\% SWPF extends the treatment and dispositioning process beyond all current and previously considered end dates. The comparisons are based on startup of CSSX-A operation at the beginning of calendar year 2006. No credit for partial closure of the HLW tank farms is taken: benefit is derived only after complete closure of both HLW tank farms. In addition, no cost savings resulting from reduced operating periods for SWPF are included in the cost benefit. Similarly, no capital or operating costs for CSSX-A have been subtracted from the cost savings. The life-cycle cost for CSSX-A (presented in Tables 12 and 13) is insignificant relative to the cost savings provided by accelerated closure of the HLW tank farms. 


\section{SCHEDULE}

The project schedule is presented as a series of Microsoft Project Gantt charts in Appendix B. The schedule is considered optimistic and should be considered a bestcase deployment. A primary basis for development of the schedule is the procurement of centrifugal contactors of existing designs. This basis significantly shortens the overall project schedule due to the elimination of a design/bid/procure cycle that may extend for up to 18 months, based on past experience.

The schedule should be viewed in terms of durations; a project start date of January 1, 2004 has been established arbitrarily.

\section{DISCUSSION AND CONCLUSIONS}

This study accomplished the following objectives: (1) establishment of a need for and the feasibility of deploying a simplified version of the SWPF CSSX system (CSSX-A), and (2) development of estimates of required capital investment and operating costs to determine benefit-to-cost ratios, which ranged from 20 to 47 depending on the comparison basis.

Eighteen million gallons of the total combined volume of supernate, interstitial liquid and dissolved salt cake wastes were identified for treatment in the proposed

system, based on reported ${ }^{137} \mathrm{Cs}$ concentrations in this waste subset that exceed the WAC for immobilization in the Saltstone facility but that are substantially less than the cesium concentration used as the design basis for SWPF CSSX.

A conceptual design of a continuous CSSX-A system was completed in this activity based on a processing rate of 3 million gallons per year and $90 \%$ availability. The primary component of the process is a 20 -stage cascade of centrifugal solvent extraction contactors. The decontamination and concentration factors were 40 and 15 , respectively. The solvent, scrub, strip, and wash solutions are to have the same compositions as planned for the SWPF CSSX system; solvent and scrub flow rates are equal. The system was designed to facilitate remote operation and direct maintenance.

Two general deployment concepts were considered: (1) deployment in an existing but unused SRS facility and (2) deployment in transportable containers. 
Consideration of these two concepts proceeded based on availability, configuration, access to needed services, proximity to HLW system and interfaces, existence of HLW system tie-ins, and cost. Deployment in three transportable containers was selected as the preferred option. It should be noted that since the deployment selection and cost/benefit analyses have been completed, the interfaces between CSSX-A and the HLW system have changed. Specifically, Tank 40 has replaced Tank 51 as the DWPF feed tank. This change has no significant impact on the cost/benefit results reported, since these results were based on a skid-mounted containerized deployment of CSSX-A. CSSX-A deployment can accommodate the change in interface tankage by relocation to a site nearer Tank 40, by installation of a transfer line to this tank, or by switching Tanks 40 and 51 back to their originally designated services.

A risk assessment indicated the $\mathrm{ES} \& \mathrm{H}$ issues that require mitigation. Because of the highly developed state of CSSX technology, there are few technical issues, none of which have the potential to make the technology unviable. Recommended development tasks to mitigate technical uncertainties are included in this report.

Significant benefits, both qualitative and quantitative in nature, will be realized by deployment of the proposed CSSX-A system. The qualitative benefits include (1) verification of full-scale contactor performance in support of the SWPF CSSX design and deployment; (2) development of a design, fabrication, installation, and operation experience base that will be at least partially applicable to the SWPF CSSX system; and (3) availability of the CSSX-A system as a means of providing contactorbased solvent extraction system operating experience. Estimates of fixed capital investment, development costs, and annual operating cost for SRS deployment (mid2003 dollars) are $\$ 9,165,200, \$ 2,734,801$, and $\$ 2,667,911$, respectively. When the economics of the CSSX-A system are compared with that of the original SRS HLW system, benefit-to-cost ratios ranging from 20 to 47 are obtained. The benefits in the cost/benefit comparison arise from expedited tank closure and avoided cost of the SWPF CSSX system. In light of these factors, timely deployment of the CSSX-A system at SRS is recommended. 


\section{REFERENCES}

1. J. K. W. Dunaway and J. R. Sessions, Waste Incidental to Reprocessing Evaluation for Disposing Saltcake to Saltstone, HLW-SDT-2001-00281, Rev. 1, Westinghouse Savannah River Company, Aiken, SC, February 2002.

2. T. J. Spears, High Level Waste Program, Savannah River Operations Office, "Salt Processing Overview: Pre-Proposal Conference," Aiken, SC, presented December $18,2001$.

3. "Liquid Waste Processing Overview," in CST Operator Training, WAAIGO01 Rev. 2, Westinghouse Savannah River Company, Aiken, SC.

4. P. V. Bonnesen, L. H. Delmau, T. J. Haverlock, and B. A. Moyer, Alkaline-Side Extraction of Cesium from Savannah River Tank Waste Using Calixarene-Crown Ether Extractant, ORNL/TM-13704, Oak Ridge National Laboratory, Oak Ridge, TN, 1998.

5. L. N. Klatt et al., Caustic-Side Solvent Extraction Solvent-Composition Recommendation, ORNL/TM-2001/258, Oak Ridge National Laboratory, Oak Ridge, TN, 2001.

6. Personal communication from L. H. Delmau, ORNL, to J. F. Birdwell, ORNL, August 21, 2003.

7. S. G. Campbell, M. W. Geeting, C. W. Kennell, J. D. Law, R. A. Leonard, M A. Norato, R. A. Pierce, T. A. Todd, D. D. Walker, and W. R. Wilmarth, Demonstration of Caustic-Side Solvent Extraction with Savannah River Site High Level Waste, WSRC-TR-2001-00223, Westinghouse Savannah River Company, Aiken, SC, 2001.

8. J. F. Birdwell, Jr., and K. K. Anderson, Evaluation of 5-cm Centrifugal Contactor Hydraulic and Mass Transfer Performance for Caustic-Side Solvent Extraction of Cesium, ORNL/TM-2001-137, Oak Ridge National Laboratory, Oak Ridge, TN, 2001.

9. J. Douglas, Conceptual Design of Chemical Processes, McGraw-Hill, New York, 1988.

10. W. Krause, Budgetary Quote from Mott Corporation, Farmington, CT, July 2003.

11. J. McDaniel, Budgetary Quote from Chempump, Warrington, PA, August 2003.

12. A. J. Palermo, Budgetary Quote from Riley Equipment, Houston, TX, August 2003.

13. B. Ewton, Budgetary Quote from International Equipment, Chattanooga, TN, August 2003. 
14. M. Peters, K. D. Timmerhaus, M. S. Peters, and R. E. West, Plant Design and Economic Analysis for Chemical Engineers, McGraw-Hill, New York, 2003.

15. J. Arnerich, Budgetary Quote from Container Solutions Inc., Walnut Creek, CA, August 2003. 
APPENDIX A

DOSE RATE CALCULATIONS 


\title{
DOSE RATE CALCULATIONS
}

\author{
Estimated Dose Rates for the SRS Radiation Waste Shipping Container
}

Dose rates at selected positions around the waste shipping container were calculated using the point kernel method. The cylindrical containers were approximated by equal-volume parallelepipeds, since such geometries are the only ones allowed in the selected point kernel code, the PUTZ ${ }^{1}$ code. The twenty-six source volumes and the eighteen detector positions are identified in Figure 1. With the origin as indicated in Figure 1, the detector locations are shown in Table 1. The detectors are located at points where there was expected to be a local maximum in the dose-rate field. The photon spectrum that results from ${ }^{137} \mathrm{Cs}$ is grouped in the 23 -group structure of the DABL $69^{2}$ cross-section library, and the data are given in Table 2. Dose rates were calculated for three subcases of the Cases A and B. The subcases included: (1) no shielding, (2) $5.08 \mathrm{~cm}$ of lead, and (3) $10.16 \mathrm{~cm}$ of lead. Detector positions are at least $10.2 \mathrm{~cm}$ from the container surface so that they are located outside the thickest shield considered. The calculated dose rates are given in Table 3 along with the estimated lead shield thickness required to reduce the dose rate to $2.5 \mathrm{mrem} / \mathrm{h}$. Indications are that $8.1 \mathrm{~cm}(3.2 \mathrm{in}$.) of lead would be sufficient shielding. Contributions to the dose rate at each detector from the various sources are given in Tables 4-9.

Results for three of the sources for the unshielded container were checked using the QADS ${ }^{3}$ point kernel computer code. Values for some detectors agreed within a few percent. Others differed by as much as a factor of 4.0 due to differences in the build-up factor treatment. QADS used build-up factors for a single, user-selected material while PUTZ used composite build-up factors. The results should be considered quite approximate. Except for line-of-sight effects, the point kernel method does not consider interactions among the various sources and shields. And radiation traveling shorter paths through the shield might make greater contributions to the dose rate at the detector positions than that calculated by the point kernel method. Evidence of this

may be seen for detector 7 (Case A) in Table 3. Normally, the $10.2 \mathrm{~cm}$ of lead should reduce the flux from ${ }^{137} \mathrm{Cs}$ photons by a factor of $10^{5}$, but the dose rate is reduced by a factor of $6 \times 10^{6}$. To be conservative, one should increase the shielding thickness to $12.7 \mathrm{~cm}(5 \mathrm{in}$.).

\section{REFERENCES}

1. D. T. Ingersoll, "User's Manual for PUTZ: A Point-Kernel Photon Shielding Code," ORNL/TM-9803 (May 1986).

2. D. T. Ingersoll, et al., "DABL69: A Broad-Group Neutron/Photon Cross-Section Library for Defense Nuclear Applications," ORNL/TM-10568 (June 1989).

3. B. L. Broadhead, "QADS: A Multidimensional Point Kernel Analysis Module," NUREG/CR-5468 and ORNL/CSD/TM-270 (May 1990). 


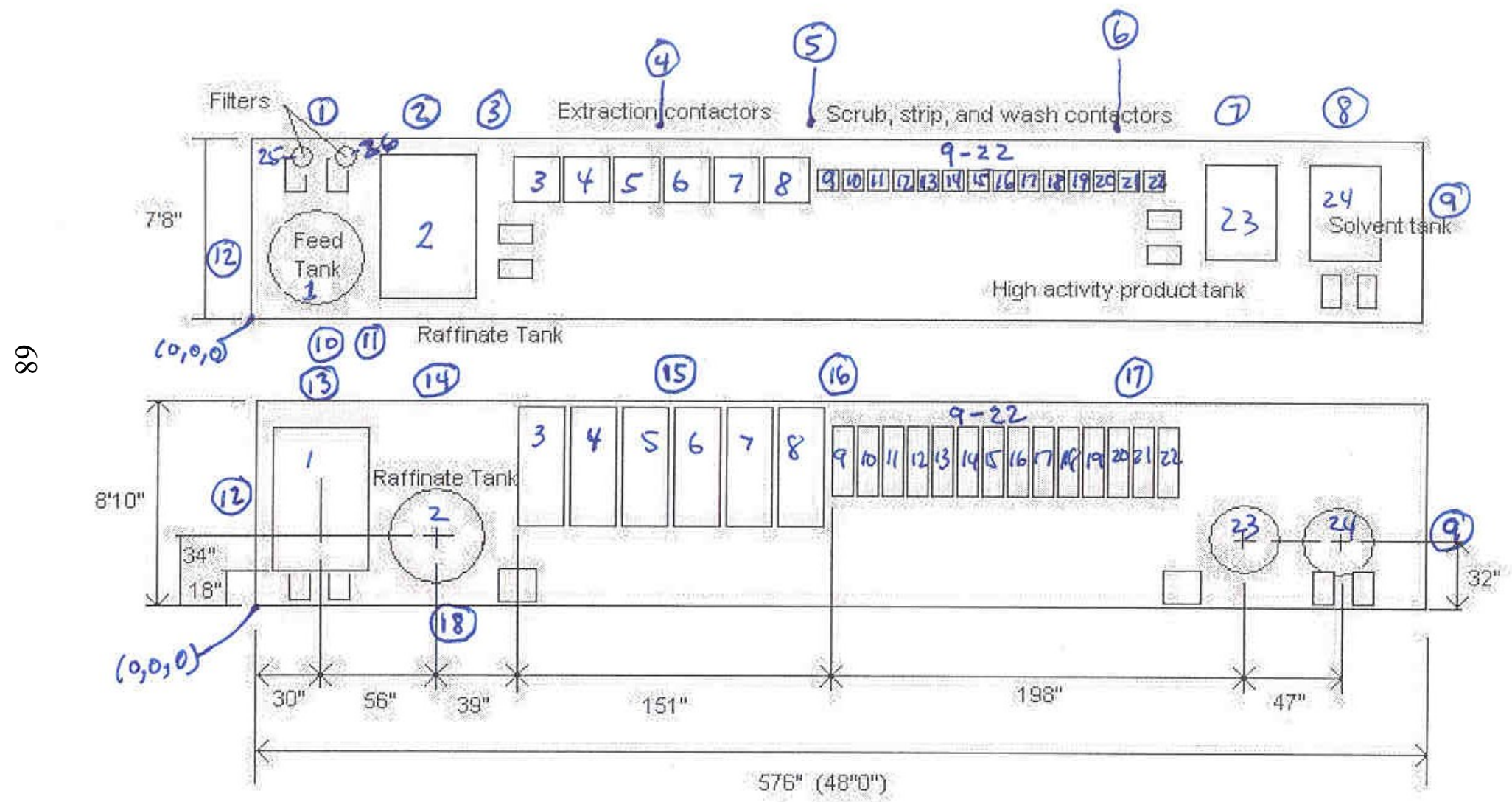

Figure 1. Source numbers and detector positions for dose-rate calculations. 
Table 1. Coordinates for detector locations

\begin{tabular}{|l|c|c|c|}
\hline \multirow{2}{*}{ Detector } & \multicolumn{3}{|c|}{ Detector Coordinates (cm) } \\
\cline { 2 - 4 } & $\mathrm{X}$ & $\mathrm{Y}$ & $\mathrm{Z}$ \\
\hline 2 & 76.2 & 244.0 & 123.0 \\
\hline 3 & 218.0 & 244.0 & 80.0 \\
\hline 4 & 305.0 & 244.0 & 173.5 \\
\hline 5 & 500.0 & 244.0 & 173.5 \\
\hline 6 & 684.6 & 244.0 & 180.0 \\
\hline 7 & 1067.0 & 244.0 & 180.0 \\
\hline 8 & 1204.0 & 244.0 & 77.0 \\
\hline 9 & 1323.0 & 244.0 & 60.0 \\
\hline 10 & 1474.0 & 142.0 & 60.0 \\
\hline 11 & 76.2 & -10.5 & 123.0 \\
\hline 12 & 147.0 & -10.5 & 100.0 \\
\hline 13 & -10.5 & 61.0 & 123.0 \\
\hline 14 & 76.2 & 61.0 & 279.5 \\
\hline 15 & 218.0 & 117.0 & 279.5 \\
\hline 16 & 500.0 & 180.0 & 279.5 \\
\hline 17 & 684.5 & 180.0 & 279.5 \\
\hline 18 & 1067.0 & 180.0 & 279.5 \\
\hline
\end{tabular}


Table 2. Photon spectrum per Curie of ${ }^{137}$ Cs.

\begin{tabular}{|l|c|c|}
\hline Group & Group Upper Energy $(\mathrm{eV})$ & Photon Source $\left(\mathrm{s}^{-1} \cdot \mathrm{Ci}^{-1}\right)$ \\
\hline 1 & $2.0+7^{\mathrm{a}}$ & 0.0 \\
\hline 2 & $1.4+7$ & 0.0 \\
\hline 3 & $1.2+7$ & 0.0 \\
\hline 4 & $1.0+7$ & 0.0 \\
\hline 5 & $8.0+6$ & 0.0 \\
\hline 6 & $7.0+6$ & 0.0 \\
\hline 7 & $6.0+6$ & 0.0 \\
\hline 8 & $5.0+6$ & 0.0 \\
\hline 9 & $4.0+6$ & 0.0 \\
\hline 10 & $3.0+6$ & 0.0 \\
\hline 11 & $2.5+6$ & 0.0 \\
\hline 12 & $2.0+6$ & 0.0 \\
\hline 13 & $1.5+6$ & $7.8805+2$ \\
\hline 14 & $1.0+6$ & $2.0678+5$ \\
\hline 15 & $7.0+5$ & $3.6046+10$ \\
\hline 16 & $4.5+5$ & $1.6690+7$ \\
\hline 17 & $3.0+5$ & $1.1136+8$ \\
\hline 18 & $1.5+5$ & $1.4023+8$ \\
\hline 19 & $1.0+5$ & $2.6747+8$ \\
\hline 20 & $7.0+4$ & $5.3644+8$ \\
\hline 21 & $4.5+4$ & $2.7000+9$ \\
\hline 22 & $3.0+4$ & $6.9184+8$ \\
\hline $23^{\mathrm{b}}$ & $2.0+4$ & $3.8253+9$ \\
\hline All & $2.0+7$ & $4.4336+10$ \\
\hline
\end{tabular}

${ }^{\mathrm{a}}$ Read as $2.0 \times 10^{7}$.

${ }^{\mathrm{b}}$ The lower energy of this group is $1.0+4 \mathrm{eV}$. 
Table 3. Dose rates at various locations around waste container.

\begin{tabular}{|c|c|c|c|c|}
\hline \multirow[b]{2}{*}{ Detector } & \multicolumn{3}{|c|}{ Dose Rate (mrem/h) } & \multirow{2}{*}{$\begin{array}{c}\text { Required Lead } \\
\text { Thickness } \\
(\mathrm{cm})^{\mathrm{a}} \\
\end{array}$} \\
\hline & No Lead & $5.08 \mathrm{~cm} \mathrm{Lead}$ & $10.16 \mathrm{~cm} \mathrm{Lead}$ & \\
\hline \multicolumn{5}{|c|}{ Case A } \\
\hline 1 & 52670 & 11.83 & $7.081-3$ & 6.1 \\
\hline 2 & 16520 & 0.739 & $3.993-4$ & 4.5 \\
\hline 3 & 13580 & 0.1411 & $2.920-5$ & 3.8 \\
\hline 4 & 13120 & 0.2951 & $1.414-4$ & 4.1 \\
\hline 5 & 20260 & 0.2255 & $9.139-5$ & 4.0 \\
\hline 6 & 134500 & 6.028 & $3.476-3$ & 5.7 \\
\hline 7 & 742200 & 204.8 & 0.1181 & 8.1 \\
\hline 8 & 109800 & 0.3128 & $2.25-8$ & 4.3 \\
\hline 9 & 7025 & 2.235 & $1.943-6$ & 5.0 \\
\hline 10 & 82530 & 145.3 & 7.974-3 & 7.2 \\
\hline 11 & 45900 & 3.522 & $1.436-3$ & 5.3 \\
\hline 12 & 90900 & 13.89 & $7.834-3$ & 6.2 \\
\hline 13 & 37740 & 10.39 & $6.605-3$ & 6.1 \\
\hline 14 & 22390 & 1.401 & $5.663-4$ & 4.8 \\
\hline 15 & 5077 & 0.184 & $1.232-4$ & 3.8 \\
\hline 16 & 11280 & 0.159 & $9.831-5$ & 3.8 \\
\hline 17 & 63610 & 6.900 & $2.321-3$ & 5.7 \\
\hline 18 & 20390 & 0.759 & $4.002-4$ & 4.5 \\
\hline \multicolumn{5}{|c|}{ Case B } \\
\hline 1 & 60910 & 11.85 & $7.085-3$ & 6.1 \\
\hline 2 & 92000 & 14.59 & $7.976-3$ & 6.3 \\
\hline 3 & 29250 & 0.695 & $1.930-4$ & 4.5 \\
\hline 4 & 20320 & 0.4504 & $2.112-4$ & 4.3 \\
\hline 5 & 23790 & 0.3211 & $1.289-4$ & 4.2 \\
\hline 6 & 140100 & 8.183 & 4.719-3 & 5.9 \\
\hline 7 & 747600 & 204.8 & 0.1181 & 8.1 \\
\hline 8 & 135000 & 7.574 & $4.659-3$ & 5.8 \\
\hline 9 & 15800 & 6.113 & $5.193-3$ & 5.7 \\
\hline 10 & 93140 & 14.56 & $7.976-3$ & 6.3 \\
\hline 11 & 77550 & 6.045 & $2.439-3$ & 5.7 \\
\hline 12 & 90910 & 13.89 & 7.834-3 & 6.2 \\
\hline 13 & 42690 & 10.57 & $6.631-3$ & 6.1 \\
\hline 14 & 42920 & 9.719 & $6.459-3$ & 6.0 \\
\hline 15 & 7709 & 0.2711 & $1.735-4$ & 4.0 \\
\hline 16 & 12560 & 0.2223 & $1.360-4$ & 4.0 \\
\hline 17 & 66150 & 7.415 & $2.747-3$ & 5.8 \\
\hline 18 & 107000 & 14.65 & $7.981-3$ & 6.3 \\
\hline
\end{tabular}

${ }^{\mathrm{a}}$ For a dose rate of $2.5 \mathrm{mrem} / \mathrm{h}$. 
Table 4. Dose rates $(\mathrm{rem} / \mathrm{h})$ for SRS Rad waste container Case A (no lead)

\begin{tabular}{|c|c|c|c|c|c|c|c|c|c|c|}
\hline & 1 & 2 & 3 & 4 & 5 & 6 & $r$ & 8 & 9 & \\
\hline 1 & +01 & $2131 \mathrm{E}+00$ & +00 & $1.1561 \mathrm{E}+\odot \odot$ & 1 & & & & & \\
\hline 2 & 4. $3205 \mathrm{E}-01$ & $3.9675 \mathrm{E}+\odot \odot$ & $8.0788 \mathrm{E}-01$ & $3.4153 \mathrm{E}-01$ & $1.4769 \mathrm{E}-01$ & $4.6242 \mathrm{E}-02$ & $3.3649 \mathrm{E}-02$ & $2.6647 \mathrm{E}-02$ & $3.7961 \mathrm{E}-09$ & $5.5778 \mathrm{E}-01$ \\
\hline 3 & $2.1770 \mathrm{E}-02$ & $8.1128 \mathrm{E}-02$ & $761 F_{-}-01$ & $.6332 \mathrm{E}-02$ & $9.9025 \mathrm{E}-03$ & $8.7059 \mathrm{E}-04$ & $3510 E-03$ & $8073 \mathrm{E}-03$ & $1.8916 \mathrm{E}-09$ & \\
\hline 4 & $1.4827 \mathrm{E}-02$ & 4.5445E- - 2 & $1.4110 \mathrm{E}-01$ & $1.6961 \mathrm{E}-01$ & $1.6727 \mathrm{E}-02$ & $1.1485 E-03$ & $7043 E-03$ & $.046 \odot E-\odot 3$ & $35 E-\odot 9$ & \\
\hline 5 & 1. $0798 \mathrm{E}-\odot 2$ & $2.7248 \mathrm{E}-\odot 2$ & $6.4471 \mathrm{E}-02$ & $4.2983 \mathrm{E}-01$ & $3.3195 E-\odot 2$ & $1.5831 \mathrm{E}-03$ & $3.1527 \mathrm{E}-03$ & $2.3362 \mathrm{E}-03$ & 1. 2786E- $\odot 8$ & $649 E-03$ \\
\hline 6 & $8.2474 \mathrm{E}-03$ & $1.7583 \mathrm{E}-\odot 2$ & $2.7568 \mathrm{E}-02$ & $4.2555 \mathrm{E}-01$ & 7.5852E- - 02 & $2.2655 \mathrm{E}-03$ & $254 E-03$ & $2.6945 \mathrm{E}-03$ & 3. $2819 E-08$ & $778 E-04$ \\
\hline 7 & $\mathrm{E}-03$ & $\mathrm{E}-02$ & & $E-01$ & $7 E-01$ & $\bar{E}-03$ & E- -3 & & & \\
\hline 8 & $5.0702 \mathrm{E}-03$ & $8.8516 \mathrm{E}-03$ & $\mathrm{E}-03$ & $7.5614 \mathrm{E}-02$ & $4.3178 \mathrm{E}-01$ & $E-\odot 2$ & $\mathrm{E}-03$ & $3 E-03$ & $7 E-07$ & \\
\hline 9 & $1.2754 \mathrm{E}-03$ & $2.4272 \mathrm{E}-03$ & $4.0443 \mathrm{E}-03$ & $2.2307 \mathrm{E}-02$ & $2.2188 \mathrm{E}-01$ & $6 \mathrm{E}-03$ & $9 \mathrm{E}-03$ & $2.3785 \mathrm{E}-03$ & $3 E-06$ & $5 E-04$ \\
\hline 10 & $E-\odot 4$ & $E-\Theta 3$ & $\mathrm{E}-03$ & $3 E-02$ & $3 \mathrm{E}-01$ & $E-03$ & E- 03 & $\mathrm{E}-03$ & -06 & $1 \mathrm{E}-$ \\
\hline 11 & $E-04$ & $E-\odot 3$ & $\mathrm{E}-03$ & $\mathrm{E}-02$ & $E-02$ & $\mathrm{E}-03$ & $E-\odot 3$ & $\odot 3$ & 05 & \\
\hline 12 & $7.8661 E-04$ & $2.3750 \mathrm{E}-\odot 3$ & $=-03$ & $7.9702 \mathrm{E}-\odot 3$ & $6 E-02$ & $\mathrm{E}-\mathrm{-02}$ & E- -3 & $3 E-03$ & $3 E-05$ & $7 E-05$ \\
\hline 13 & $E-\odot 4$ & $E-\odot 3$ & -03 & $E-\odot 3$ & $E-\odot 2$ & $E-\odot 2$ & $E-03$ & $\mathrm{E}-03$ & $E-\odot 4$ & $8 E-04$ \\
\hline 14 & -04 & E- 03 & -03 & $\mathrm{E}-03$ & $E-02$ & -02 & $=-03$ & & & $E-\odot 4$ \\
\hline 15 & $7.3420 \mathrm{E}-04$ & $E-03$ & $5 E-03$ & OE- -03 & $5 E-02$ & $\mathrm{E}-02$ & $\mathrm{E}-\mathrm{-02}$ & BE- -3 & $E-03$ & $1 E-04$ \\
\hline 16 & -94 & -03 & -04 & $E-03$ & $\mathrm{E}-\odot 2$ & $=-02$ & $=-02$ & & & : 01 \\
\hline 17 & $E-04$ & E- 03 & -04 & E- -3 & $E-\odot 2$ & $E-\odot 2$ & $E-02$ & & & $9 E-\odot 4$ \\
\hline 18 & $5.2876 \mathrm{E}-\odot 4$ & 1. $2309 \mathrm{E}-\odot 3$ & $6.8925 E-\odot 4$ & $8 E-\odot 3$ & 7E- - & $4 E-01$ & $7 E-\odot 2$ & $3 E-\odot 3$ & 1. $2251 \mathrm{E}-03$ & $1 E-\odot 4$ \\
\hline 19 & $-\odot 4$ & 1.1 & 5.8 & $\mathrm{E}-03$ & $\mathrm{E}-03$ & $\mathrm{E}-01$ & -02 & & & $7 F_{-} \cap 1$ \\
\hline 20 & $\mathrm{E}-03$ & -02 & -03 & $\mathrm{E}-02$ & $E-02$ & $E+\odot \odot$ & 01 & 91 & 02 & $B \mathrm{E}$ \\
\hline 21 & $5.9260 \mathrm{E}-03$ & 1. $3493 \mathrm{E}-02$ & $\partial E-03$ & $1.7930 \mathrm{E}-\odot 2$ & $3 E-02$ & $46 \mathrm{E}+\odot \odot$ & $7 E-01$ & $4 E-01$ & $6 \mathrm{E}-02$ & $3.1089 \mathrm{E}-03$ \\
\hline 22 & $3.5976 \mathrm{E}-04$ & $E-04$ & E- -04 & $3 E-03$ & E- -03 & $E-01$ & $E-02$ & 02 & E- 03 & $6 \mathrm{E}-04$ \\
\hline 23 & $3.7986 \mathrm{E}+\odot \odot$ & $5.0087 \mathrm{E}+\odot \odot$ & $3 \mathrm{E}+0 \odot$ & $2+01$ & $3 \mathrm{E}+01$ & $\mathrm{E}+02$ & $\mathrm{E}+\mathrm{O} 2$ & +02 & $E+\Theta \odot$ & \\
\hline 24 & $\odot . \odot \odot \odot \odot E+\odot \odot$ & $\odot .0 \odot \odot \odot E+\odot \odot$ & $\odot . \odot \odot \odot \odot E+\odot \odot$ & $\odot .0 \odot \odot \odot E+\odot \odot$ & $\odot .0 \odot \odot \odot E+\odot \odot$ & $\partial \mathrm{E}+0 \odot$ & $\odot \mathrm{E}+\odot \odot$ & $\odot \mathrm{E}+\odot \odot$ & $\odot E+\odot \odot$ & $\odot \odot E+\odot \odot$ \\
\hline 25 & $1.6367 \mathrm{E}+01$ & $1.0803 \mathrm{E}+0 \odot$ & E- 01 & $1 E-\odot 2$ & $7 E-02$ & $E-03$ & $\mathrm{FE}-03$ & $5 E-03$ & $2.8347 \mathrm{E}-14$ & $7.8-8-2.8$ \\
\hline 26 & $\mathrm{E}+01$ & $\mathrm{E}+\Theta \Theta$ & E-01 & & & & & & & \\
\hline & $5.2664 \mathrm{E}+01$ & $1.6517 \mathrm{E}+01$ & $1.3580 \mathrm{E}+01$ & $1.3117 \mathrm{E}+01$ & 2. $0259 \mathrm{E}+01$ & $\mathrm{E}+\mathrm{O} 2$ & $9 \mathrm{E}+02$ & $1.0977 \mathrm{E}+02$ & 7. $0248 \mathrm{E}+0 \Theta$ & 8.2 \\
\hline
\end{tabular}


Table 4 (continued). Dose rates $(\mathrm{rem} / \mathrm{h}$ ) for SRS Rad waste container Case A (no lead)

\begin{tabular}{|c|c|c|c|c|c|c|c|c|}
\hline & 11 & 12 & 13 & 14 & 15 & 16 & & \\
\hline 1 & $3.9740 \mathrm{E}+01$ & $563 E+01$ & $3.3249 \mathrm{E}+01$ & $4516 E+01$ & $7 \mathrm{E}+0 \odot$ & 7. $0360 \mathrm{E}-01$ & $33 E-01$ & $1 \mathrm{E}+0 \odot$ \\
\hline 2 & $1.6644 \mathrm{E}+\odot \odot$ & $4465 E-\odot 4$ & $.5825 \mathrm{E}-01$ & $\odot 762 E+\odot \odot$ & $2629 E-01$ & 3979E- - 22 & 3. $0790 \mathrm{E}-\odot 2$ & $4.5546 \mathrm{E}+\odot \odot$ \\
\hline 3 & 3 & & 12 & $2 \mathrm{~F}-02$ & & & & \\
\hline 4 & $2.4371 \mathrm{E}-04$ & $.3683 E-06$ & $1.6059 \mathrm{E}-02$ & $4.2462 \mathrm{E}-02$ & $3340 E-02$ & 16E-03 & $69 \mathrm{E}-05$ & 1 \\
\hline 5 & $8.5206 \mathrm{E}-05$ & $3.6623 E-06$ & 1. $0884 \mathrm{E}-\odot 2$ & $2.3633 \mathrm{E}-\odot 2$ & $1.5390 \mathrm{E}-01$ & $1.1893 \mathrm{E}-02$ & $4.1136 \mathrm{E}-05$ & $2.4114 \mathrm{E}-02$ \\
\hline 6 & כE- $\odot 4$ & $\Theta E-\odot 7$ & $51 E-03$ & $1 E-02$ & $4 E-01$ & $8 E-02$ & $15 E-\odot 4$ & $3 E-02$ \\
\hline 7 & $3 E-\odot 4$ & $E-\odot 8$ & E- $\odot 3$ & $E-\odot 2$ & $7 E-02$ & $8 \mathrm{E}-02$ & $3 E-\odot 4$ & \\
\hline 8 & $76 E-\odot 4$ & $8 \mathrm{E}-\odot 8$ & ЭE-๑3 & $33 E-03$ & $3 E-02$ & $22 \mathrm{E}-01$ & $4 E-04$ & $79 E-03$ \\
\hline 9 & $E-\odot 4$ & $6 \mathrm{E}-\odot 8$ & $29 E-03$ & $5 E-03$ & $5 E-03$ & $36 \mathrm{E}-01$ & $96 \mathrm{E}-\odot 4$ & $1 E-03$ \\
\hline 10 & $E-04$ & $\mathrm{E}-\odot 8$ & E- 03 & $\mathrm{E}-\odot 3$ & $\mathrm{E}-03$ & $E-02$ & $E-\odot 4$ & $E-\odot 3$ \\
\hline 11 & $E-\odot 4$ & E-๑8 & $E-03$ & $E-03$ & $7 E-\odot 3$ & $\odot 2$ & $3 E-03$ & $-\odot 3$ \\
\hline 12 & $E-04$ & $E-\odot 8$ & $E-\odot 3$ & $E-\odot 3$ & E- $\odot 3$ & $E-02$ & $4 \mathrm{E}-\odot 3$ & $28 E-03$ \\
\hline 13 & $E-03$ & E- ๑८ & $E-\odot 3$ & $E-\odot 3$ & {$[01$} & $E-02$ & $\mathrm{E}-\odot 3$ & E- -03 \\
\hline 14 & $1.6314 \mathrm{E}-03$ & $1.4451 \mathrm{E}-\odot 8$ & כE- $\odot 4$ & $5 E-03$ & $5.2107 \mathrm{E}-04$ & $8 \mathrm{E}-03$ & $2 E-03$ & $07 E-\odot 3$ \\
\hline 15 & $=02$ & $F_{-} \mathrm{O}$ & $E-84$ & & & & $-\odot 2$ & $=-03$ \\
\hline 0 & $E-03$ & E- ๑९ & $\mathrm{E}-\odot 4$ & $E-\odot 4$ & E- 05 & $E-03$ & $\mathrm{E}-\mathrm{-02}$ & $\mathrm{E}-03$ \\
\hline 17 & $3 E-03$ & $E-\odot 9$ & $4 E-\odot 4$ & $3 E-\odot 4$ & $8 E-05$ & $9 E-04$ & $E-\odot 2$ & $8 E-03$ \\
\hline 18 & $E-03$ & $\mathrm{E}-\odot 9$ & $E-\odot 4$ & E- & E- 05 & $\mathrm{E}-03$ & -02 & $0 E-03$ \\
\hline 19 & 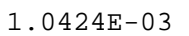 & 9 & & & & & & \\
\hline 20 & $\mathrm{E}-02$ & E- -8 & $\mathrm{E}-03$ & $E-03$ & $\mathrm{E}-04$ & $5 E-03$ & $9 \mathrm{E}+0 \odot$ & $5 E-02$ \\
\hline 21 & $8 \mathrm{E}-02$ & $1 \mathrm{E}-07$ & $2 \mathrm{E}-03$ & $5 E-03$ & $1.5413 \mathrm{E}-04$ & $1 E-03$ & $72 \mathrm{E}+0 \odot$ & 1. $8043 \mathrm{E}-02$ \\
\hline 22 & 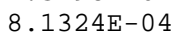 & \ulcorner & - & & & & $\mathrm{F}-\odot 2$ & $18 E-03$ \\
\hline 23 & 4.2 & $F-\rho 7$ & 3. & $5+00$ & $E+\odot \odot$ & & & $2 E+\odot \odot$ \\
\hline 24 & $\Theta E+\odot \odot$ & $\odot \mathrm{E}+\Theta \odot$ & $\odot E+\odot \odot$ & $\odot \mathrm{E}+\odot \odot$ & $\vartheta \odot \odot E+\odot \odot$ & $\odot . \odot \odot \odot \odot E+\odot \odot$ & $\odot . \odot \odot \odot \odot E+\odot \odot$ & $\odot . \odot \odot \odot \odot E+\odot \odot$ \\
\hline 25 & $E-04$ & $E-01$ & $E-\odot 2$ & & & & -06 & 9.1 \\
\hline 26 & $2.0562 \mathrm{E}-01$ & $1.5929 \mathrm{E}-02$ & $8.3953 \mathrm{E}-02$ & $7.5945 \mathrm{E}-01$ & $2.4108 \mathrm{E}-02$ & $3.6218 \mathrm{E}$ & 1. $0979 \mathrm{E}-05$ & 6. $2036 \mathrm{E}-01$ \\
\hline
\end{tabular}
$\begin{array}{lllllllll}\text { total } & 4.5901 \mathrm{E}+01 & 9.0900 \mathrm{E}+01 & 3.7738 \mathrm{E}+01 & 2.2387 \mathrm{E}+01 & 5.0760 \mathrm{E}+00 & 1.1283 \mathrm{E}+01 & 6.3610 \mathrm{E}+01 & 2.0389 \mathrm{E}+01\end{array}$ 
Table 5. Dose rates $(\mathrm{rem} / \mathrm{h})$ for SRS Rad waste container Case A $(5.08 \mathrm{~cm}$ lead)

\begin{tabular}{|c|c|c|c|c|c|c|c|c|c|c|}
\hline & 1 & 2 & 3 & 4 & 5 & 6 & 7 & 8 & 9 & 0 \\
\hline 1 & & & & 8 & & 5 & & & & \\
\hline 2 & $1.1632 \mathrm{E}-06$ & $7.2912 \mathrm{E}-\odot 4$ & $2.7430 \mathrm{E}-05$ & 4. 8913E-०7 & 1. $2772 \mathrm{E}-\odot 9$ & $5.3596 \mathrm{E}-15$ & $2.3481 \mathrm{E}-16$ & 3. $0223 \mathrm{E}-17$ & $.6760 \mathrm{E}-14$ & $1.5395 \mathrm{E}-06$ \\
\hline 3 & $1.6833 \mathrm{E}-14$ & $1.5893 E-\odot 9$ & $6.5594 \mathrm{E}-05$ & $2.8008 \mathrm{E}-\odot 9$ & 7. $0051 \mathrm{E}-17$ & $3.6340 \mathrm{E}-19$ & $2.5324 \mathrm{E}-19$ & $.9492 \mathrm{E}-19$ & $8255 E-14$ & $769 \mathrm{E}$ \\
\hline 4 & $2.3206 \mathrm{E}-16$ & $9 E-12$ & $78 E-\odot 7$ & $39 E-\odot 6$ & $4.7209 E-15$ & $360 E-19$ & ००E-19 & $46 \mathrm{E}-19$ & $840 E-13$ & \\
\hline 5 & 1. $0247 \mathrm{E}-17$ & $3.5821 \mathrm{E}-14$ & $5.9456 \mathrm{E}-10$ & $1.4934 \mathrm{E}-04$ & 1. $5315 \mathrm{E}-12$ & $5.2614 \mathrm{E}-19$ & $3.4278 \mathrm{E}-19$ & $2.5373 E-19$ & $2.9925 \mathrm{E}-13$ & \\
\hline 6 & 1. $3483 \mathrm{E}-18$ & $4.5266 \mathrm{E}-16$ & $2.8113 \mathrm{E}-13$ & $1.4285 \mathrm{E}-\odot 4$ & 2. 3284E-๑9 & $6.5252 \mathrm{E}-19$ & $4.0621 \mathrm{E}-19$ & $2.9349 \mathrm{E}-19$ & $8.2972 \mathrm{E}-13$ & 937E-12 \\
\hline 7 & $7.2161 \mathrm{E}-19$ & $1.8223 \mathrm{E}-17$ & $9.8387 \mathrm{E}-16$ & $61 E-06$ & 1. $0515 E-06$ & $3.7811 \mathrm{E}-19$ & $6 \mathrm{E}-19$ & $1322 E-19$ & 12 & \\
\hline 8 & $5.6103 \mathrm{E}-19$ & $1.7997 \mathrm{E}-18$ & $2.2893 \mathrm{E}-17$ & $6 E-\odot 9$ & 1. 4077E-๑4 & $5.3822 \mathrm{E}-18$ & $2 \mathrm{E}-19$ & $2 E-19$ & $7 E-12$ & 13 \\
\hline 9 & $2.2640 \mathrm{E}-19$ & $3.6204 \mathrm{E}-19$ & 1. $2666 \mathrm{E}-18$ & $7.4592 \mathrm{E}-13$ & $7.5467 \mathrm{E}-05$ & $9.9825 \mathrm{E}-18$ & LEE-19 & $21 E-19$ & $5.9549 \mathrm{E}-11$ & $1.8206 \mathrm{E}-15$ \\
\hline 10 & 2. $0658 \mathrm{E}-19$ & $\mathrm{E}-19$ & $\mathrm{E}-19$ & $8 \mathrm{E}-14$ & $5 E-06$ & $3 \mathrm{E}-17$ & $8 \mathrm{E}-19$ & $7 \mathrm{E}-19$ & $\mathrm{OE}-10$ & $9 E-16$ \\
\hline 11 & $E-19$ & $E-19$ & $\mathrm{E}-19$ & $3 E-15$ & $2 E-\odot 7$ & $E-16$ & $\mathrm{E}-19$ & $E-19$ & & \\
\hline 12 & $1.7397 \mathrm{E}-19$ & $2.6017 \mathrm{E}-19$ & อE-19 & $1.4928 \mathrm{E}-16$ & 1. $9949 \mathrm{E}-\odot 8$ & $1 E-15$ & $4 E-18$ & $3 E-19$ & 7.1 & 17 \\
\hline 13 & $1.6047 \mathrm{E}-19$ & $E-19$ & $E-19$ & $6 \mathrm{E}-17$ & $6 \mathrm{E}-10$ & $E-14$ & $\mathrm{E}-18$ & $E-19$ & $\odot 8$ & $9 \mathrm{E}-17$ \\
\hline 14 & $1.4846 \mathrm{E}-19$ & $E-19$ & $E-19$ & $3 E-18$ & $8 \mathrm{E}-11$ & $E-12$ & $\mathrm{E}-17$ & $E-19$ & & $3 E-17$ \\
\hline 15 & 1. $3775 \mathrm{E}-19$ & $1.9699 \mathrm{E}-19$ & $2.6537 \mathrm{E}-19$ & $1 \mathrm{E}-18$ & $4.8239 E-13$ & E-10 & $8 E-16$ & $3 E-19$ & $4 E-07$ & $6 \mathrm{E}-17$ \\
\hline 16 & 1.281 & E-19 & $\mathrm{E}-19$ & $\mathrm{E}-19$ & $E-14$ & $E-\odot 9$ & $E-16$ & & & $\mathrm{E}-17$ \\
\hline 17 & $E-19$ & $E-19$ & $E-19$ & $E-19$ & $E-15$ & $E-\odot 7$ & 15 & 18 & 97 & $8 E-17$ \\
\hline 18 & $1.1170 \mathrm{E}-19$ & 1. $5412 \mathrm{E}-19$ & $4 E-19$ & 91E-19 & $1.5737 \mathrm{E}-16$ & $2.1477 \mathrm{E}-06$ & $7 \mathrm{E}-14$ & $9 \mathrm{E}-17$ & $2 E-\odot 7$ & $5 E-18$ \\
\hline 19 & $3 E-19$ & $E-19$ & $E-19$ & $1 E-19$ & $4 \mathrm{E}-17$ & E- 05 & $\mathrm{E}-13$ & 17 & 4. & $6 \mathrm{E}-18$ \\
\hline 20 & $=-18$ & 1.9 & $E-18$ & $E-18$ & 6.0 & -03 & -10 & & & -17 \\
\hline 21 & $3 E-18$ & $E-18$ & $E-18$ & $E-18$ & $99 \mathrm{E}-17$ & $4 E-\odot 3$ & $6 E-09$ & 3. & 1.2 & $2 \mathrm{E}-17$ \\
\hline 22 & $8.7008 \mathrm{E}-20$ & $E-19$ & $2 E-19$ & $1 E-19$ & $5.9371 \mathrm{E}-19$ & $2.9847 \mathrm{E}-05$ & 1. 5123E-๑9 & $21 E-14$ & $1 E-\odot 6$ & $6.5421 \mathrm{E}-19$ \\
\hline 23 & $=-16$ & 1.1 & 2.2 & & $\mathrm{E}-10$ & E- -4 & $\mathrm{E}-01$ & & & \\
\hline 24 & $E+\odot \odot$ & $E+0 \odot$ & $\odot . \odot$ & $E+\odot \odot$ & $E+\odot \odot$ & $E+\odot \odot$ & $E+\odot \odot$ & & $\odot$. & 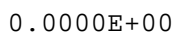 \\
\hline 25 & $4.8085 E-03$ & 1. $0111 \mathrm{E}-10$ & $9.5990 \mathrm{E}-15$ & $2.6342 \mathrm{E}-17$ & 1. $3232 \mathrm{E}-17$ & $5.1150 \mathrm{E}-18$ & $3.9790 \mathrm{E}-18$ & 49E-18 & $5 E-18$ & $94 \mathrm{E}-08$ \\
\hline 26 & $36 E-04$ & $E-06$ & & & $1.5824 \mathrm{E}-$ & $\mathrm{E}-18$ & $2 E-18$ & $7 \mathrm{E}-18$ & & \\
\hline & $1.1826 \mathrm{E}-02$ & 7.3901E-04 & $1.4106 \mathrm{E}-04$ & $2.9507 \mathrm{E}-\odot 4$ & $2.2553 \mathrm{E}-\odot 4$ & $6.0282 \mathrm{E}-03$ & $2.0474 \mathrm{E}-01$ & $3.1276 \mathrm{E}-04$ & $2.2354 \mathrm{E}-03$ & 1. $4529 \mathrm{E}-$ \\
\hline
\end{tabular}


Table 5 (continued). Dose rates $(\mathrm{rem} / \mathrm{h})$ for SRS Rad waste container case A $(5.08 \mathrm{~cm}$ lead)

\begin{tabular}{|c|c|c|c|c|c|c|c|c|}
\hline & 11 & 12 & 13 & 14 & 15 & 16 & & \\
\hline 1 & $3.2698 \mathrm{E}-03$ & $E-02$ & 1.037 & $8.4740 \mathrm{E}-\odot 4$ & 1. $2900 \mathrm{E}-\odot 9$ & $2.9742 \mathrm{E}-13$ & $49 E-16$ & $3 E-05$ \\
\hline 2 & 1. $3278 \mathrm{E}-\odot 4$ & $6.2528 E-\odot 9$ & $9.6171 \mathrm{E}-06$ & $4.3781 E-04$ & $6.2427 \mathrm{E}-07$ & $2.5267 \mathrm{E}-\odot 9$ & $9.1543 \mathrm{E}-14$ & 7. 3093E- $\odot 4$ \\
\hline 3 & $69 \mathrm{E}-10$ & $76 \mathrm{E}-10$ & $83 F_{1}$ & & 6 & & & \\
\hline 4 & $1.2413 \mathrm{E}-10$ & $1.2608 \mathrm{E}-10$ & $6.1373 \mathrm{E}-13$ & $1.5827 \mathrm{E}-08$ & $0 E-06$ & $9.9643 \mathrm{E}-12$ & $4.4968 \mathrm{E}-19$ & \\
\hline 5 & $6.2984 \mathrm{E}-12$ & $1.1653 \mathrm{E}-10$ & $1.5069 \mathrm{E}-14$ & $2.6002 \mathrm{E}-10$ & $8.4986 E-05$ & $9.2316 \mathrm{E}-10$ & $6.1211 \mathrm{E}-19$ & $1.5243 \mathrm{E}-07$ \\
\hline 6 & $1.8863 \mathrm{E}-12$ & $3.5355 \mathrm{E}-12$ & $7.4290 \mathrm{E}-16$ & $4.4334 \mathrm{E}-12$ & $8.5709 E-05$ & $9.4566 \mathrm{E}-\odot 8$ & $1.9721 \mathrm{E}-18$ & $59 E-\odot 8$ \\
\hline 7 & $9 E-13$ & $2 \mathrm{E}-12$ & $2 \mathrm{E}-17$ & $1 E-14$ & & & $9 E-17$ & \\
\hline 8 & $2.3030 \mathrm{E}-13$ & $1.2637 \mathrm{E}-12$ & $9.1371 \mathrm{E}-18$ & $2.2148 \mathrm{E}-15$ & $9 E-\odot 8$ & $363 E-05$ & $524 \mathrm{E}-17$ & 5०3E-10 \\
\hline 9 & $4.3558 \mathrm{E}-14$ & $2.1950 \mathrm{E}-12$ & $2.2711 \mathrm{E}-19$ & $2.1956 \mathrm{E}-18$ & $2.4292 \mathrm{E}-11$ & $90 \mathrm{E}-05$ & $1.6929 \mathrm{E}-16$ & $38 \mathrm{E}-11$ \\
\hline 10 & $E-14$ & $E-13$ & $\mathrm{E}-19$ & $3 E-19$ & $\mathrm{E}-12$ & $4 E-06$ & $5 E-16$ & \\
\hline 11 & 1. $0419 \mathrm{E}-14$ & $6.8063 \mathrm{E}-13$ & $61 E-19$ & $E-19$ & 13 & $1 E-\odot 7$ & 14 & \\
\hline 12 & $6.1219 \mathrm{E}-15$ & $5.4603 \mathrm{E}-13$ & $6 \mathrm{E}-19$ & $E-19$ & $9 \mathrm{E}-15$ & $8 E-\odot 8$ & $5 E-13$ & $2 E-12$ \\
\hline 13 & $4 \mathrm{E}-15$ & $6 \mathrm{E}-13$ & $\mathrm{E}-19$ & $E-19$ & $7 \mathrm{E}-16$ & $2 \mathrm{E}-09$ & $\mathrm{E}-12$ & $8 \mathrm{E}$ \\
\hline 14 & $2.7464 \mathrm{E}-15$ & $3.5308 E-13$ & $4 \mathrm{E}-19$ & $9 \mathrm{E}-19$ & $6 \mathrm{E}-17$ & $7 E-10$ & $E-11$ & $14 \mathrm{E}-13$ \\
\hline 15 & 1. $0415 \mathrm{E}-15$ & $9 E-13$ & $E-19$ & $E-19$ & 17 & $E-11$ & $\odot 9$ & $1 E-14$ \\
\hline 16 & -16 & $E-13$ & $E-19$ & $\mathrm{E}-19$ & $E-18$ & $1 \mathrm{E}-13$ & $6 \mathrm{E}-\odot 8$ & $3 E-14$ \\
\hline 17 & $1.8517 \mathrm{E}-16$ & $1.8562 \mathrm{E}-13$ & $3 E-19$ & $3 E-19$ & OE-19 & $4 E-14$ & $\odot \mathrm{E}-07$ & $1 E-15$ \\
\hline 18 & $8.6877 \mathrm{E}-17$ & $1.5023 \mathrm{E}-13$ & $1.1045 \mathrm{E}-19$ & $1.5601 \mathrm{E}-19$ & $2 E-19$ & $7 E-15$ & $5 E-06$ & $37 E-15$ \\
\hline 19 & $E-17$ & $E-13$ & $E-19$ & $E-19$ & $E-19$ & $E-16$ & E- 05 & $8=15$ \\
\hline 20 & $3.2486 \mathrm{E}-16$ & $7 E-12$ & $E-18$ & $E-18$ & $\mathrm{E}-18$ & $8 E-16$ & $9 E-\odot 4$ & $9 \mathrm{E}-15$ \\
\hline 21 & $1.7095 \mathrm{E}-16$ & $3.0622 \mathrm{E}-11$ & $1.3739 \mathrm{E}-18$ & 5०E-18 & $4.1307 \mathrm{E}-18$ & $26 E-16$ & $1 E-04$ & $3.2771 \mathrm{E}-15$ \\
\hline 22 & $6.1272 \mathrm{E}-18$ & $E-12$ & $E-20$ & E-19 & $3 E-19$ & $3 E-18$ & $3 E-05$ & $5 E-16$ \\
\hline 23 & $7.4207 \mathrm{E}-15$ & $2 E-12$ & $E-14$ & $E-13$ & $E-10$ & E- -07 & $9 E-03$ & $P F_{-}$ \\
\hline 24 & $\odot . \odot \odot \odot \odot E+\odot \odot$ & $\odot . \odot \odot \odot \odot E+\odot \odot$ & $\odot . \odot \odot \odot \odot E+\odot \odot$ & $\ominus \odot+\odot \odot$ & $\odot . \odot \odot \odot \odot E+\odot \odot$ & $\odot \odot E+\odot \odot$ & $\ominus \odot E+\odot \odot$ & $\odot \odot E+\odot \odot$ \\
\hline 25 & $6.8743 \mathrm{E}-09$ & $2.2745 \mathrm{E}-\odot 8$ & $2 . \odot 249 E-\odot 6$ & $3 E-05$ & $\mathrm{E}-11$ & $7 E-16$ & $4 E-18$ & $4 E-\odot 6$ \\
\hline 26 & $E-04$ & $7 \mathrm{E}-08$ & $2 E-06$ & $6 \mathrm{E}-05$ & $9 \mathrm{E}-10$ & $4 \mathrm{E}-15$ & $3 \mathrm{E}-18$ & \\
\hline & $3.5220 E-03$ & $1.3894 \mathrm{E}-02$ & 1. $0391 \mathrm{E}-02$ & $1.4007 \mathrm{E}-\odot 3$ & $6 E-04$ & $1.5904 \mathrm{E}-04$ & $\mathrm{OE}-03$ & $7.5925 \mathrm{E}-\odot 4$ \\
\hline
\end{tabular}


Table 6. Dose rates $(\mathrm{rem} / \mathrm{h})$ for SRS Rad waste container Case A (10.16 cm lead)

\begin{tabular}{|c|c|c|c|c|c|c|c|c|c|c|}
\hline & et & 2 & 3 & 4 & 5 & 6 & 7 & 8 & 9 & \\
\hline 1 & $3.97 \mathrm{~g}$ & 37 & 34 & & & 16 & 1.32 & $1.0 \varepsilon$ & 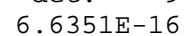 & \\
\hline 2 & $2.3643 \mathrm{E}-10$ & $3.9875 \mathrm{E}-07$ & $8.1343 \mathrm{E}-09$ & $6.7424 \mathrm{E}-12$ & $9.2239 \mathrm{E}-16$ & $1.2115 \mathrm{E}-17$ & $9.1522 \mathrm{E}-18$ & 7. $3088 \mathrm{E}-18$ & 7. $0914 \mathrm{E}-17$ & $7.1059 \mathrm{E}-11$ \\
\hline 3 & 2. $3929 \mathrm{E}-18$ & $9.1809 \mathrm{E}-16$ & $8713 E-08$ & $.8464 \mathrm{E}-15$ & $1.6313 \mathrm{E}-18$ & $3.6729 \mathrm{E}-19$ & $2.5648 \mathrm{E}-19$ & $1.9776 \mathrm{E}-19$ & $3.4812 \mathrm{E}-17$ & \\
\hline 4 & $1.6326 \mathrm{E}-18$ & $9.0431 \mathrm{E}-18$ & $2.3529 \mathrm{E}-12$ & 1. $9432 \mathrm{E}-11$ & $2.3966 \mathrm{E}-18$ & $4.3784 \mathrm{E}-19$ & $2.9649 \mathrm{E}-19$ & 2. $2449 \mathrm{E}-19$ & $9.7095 \mathrm{E}-17$ & 1 \\
\hline 5 & $1.1849 \mathrm{E}-18$ & $2.5389 \mathrm{E}-18$ & $3.8396 \mathrm{E}-16$ & $7.3300 \mathrm{E}-\odot 8$ & $5.3178 \mathrm{E}-18$ & $5.3078 \mathrm{E}-19$ & $3.4655 \mathrm{E}-19$ & $2.5697 \mathrm{E}-19$ & $2.6523 \mathrm{E}-16$ & $8.6286 \mathrm{E}$ \\
\hline 6 & $8.9753 \mathrm{E}-19$ & $1.7307 \mathrm{E}-18$ & $3.8158 \mathrm{E}-18$ & $6.8095 \mathrm{E}-\odot 8$ & $1.4956 \mathrm{E}-15$ & $6.5660 \mathrm{E}-19$ & $4.1030 \mathrm{E}-19$ & $2.9698 \mathrm{E}-19$ & $75 E-16$ & $90 E-18$ \\
\hline 7 & E-19 & $\mathrm{E}-18$ & $3 \mathrm{E}-18$ & $7 \mathrm{E}-11$ & $8 \mathrm{E}-11$ & & & & & \\
\hline 8 & $5.6458 \mathrm{E}-19$ & $9.4865 \mathrm{E}-19$ & $1.5321 \mathrm{E}-18$ & $9 E-15$ & $6.2422 \mathrm{E}-08$ & $99 E-18$ & 6 & & $7 E-15$ & \\
\hline 9 & $2.2855 \mathrm{E}-19$ & $3.6092 \mathrm{E}-19$ & $5.5131 \mathrm{E}-19$ & $2.4288 \mathrm{E}-18$ & $2.8461 \mathrm{E}-08$ & $25 \mathrm{E}-19$ & $6 \mathrm{E}-19$ & $12 \mathrm{E}-19$ & $4 \mathrm{E}-14$ & $5 \mathrm{E}-19$ \\
\hline 10 & $4 \mathrm{E}-19$ & 3. $2280 \mathrm{E}-19$ & $3 E-19$ & $E-18$ & $\mathrm{OE}-10$ & $6 \mathrm{E}-19$ & $9 \mathrm{E}-19$ & $3 E-19$ & E-13 & 19 \\
\hline 11 & 1. $9120 \mathrm{E}-19$ & $2 E-19$ & 4. $2074 \mathrm{E}-19$ & $9 \mathrm{E}-18$ & $2.8674 \mathrm{E}-12$ & $5 E-18$ & $8 E-19$ & $7 E-19$ & $E-12$ & 19 \\
\hline 12 & $1.7586 \mathrm{E}-19$ & $2.6243 \mathrm{E}-19$ & $3.7212 \mathrm{E}-19$ & $9.6555 \mathrm{E}-19$ & $1.8427 \mathrm{E}-14$ & $4 E-18$ & $6 E-19$ & $98 E-19$ & $1 E-12$ & $8 E-19$ \\
\hline 13 & $E-19$ & $E-19$ & $E-19$ & LE-19 & $6 E-16$ & $8 E-18$ & DE-19 & $2 E-19$ & $3 E-11$ & $2 \mathrm{E}-19$ \\
\hline 14 & $0 \mathrm{E}-19$ & $5-19$ & E-19 & $E-19$ & $4 \mathrm{E}-17$ & $4 \mathrm{E}-18$ & $7 E-19$ & $7 E-19$ & $E-10$ & $9 E-19$ \\
\hline 15 & 1. $3942 \mathrm{E}-19$ & $0 \mathrm{E}-19$ & $9 \mathrm{E}-19$ & $8 \mathrm{E}-19$ & $6 \mathrm{E}-18$ & $4 \mathrm{E}-17$ & $4 \mathrm{E}-19$ & $\mathrm{OE}-19$ & -10 & -19 \\
\hline 16 & 1.2 & $E-19$ & $E-19$ & $8 E-19$ & $6 \mathrm{E}-18$ & $\mathrm{E}-15$ & $\mathrm{E}-18$ & $E-19$ & $E-10$ & $2 \mathrm{E}-19$ \\
\hline 17 & 1.2 & -19 & $E-19$ & $4 \mathrm{E}-19$ & $E-18$ & $7 E-13$ & $3 E-18$ & OE -19 & E-10 & $2 \mathrm{E}-19$ \\
\hline 18 & $1.1321 \mathrm{E}-19$ & $1.5589 \mathrm{E}-19$ & 2. $0166 \mathrm{E}-19$ & 3. $9040 \mathrm{E}-19$ & $9.7808 \mathrm{E}-19$ & $5 E-11$ & $9 E-18$ & $52 E-19$ & $8 E-10$ & $5 E-19$ \\
\hline 19 & 1.0 & 1.4 & $E-19$ & $6 \mathrm{E}-19$ & $\mathrm{E}-19$ & $7 E-09$ & $E-18$ & OE -19 & $E-10$ & $3 E-19$ \\
\hline 20 & $4 E-18$ & 2.0 & $\mathrm{E}-18$ & $4 E-18$ & $E-17$ & $\mathrm{HE}-06$ & F-16 & OE -17 & $E-\odot 9$ & $8 \mathrm{E}-18$ \\
\hline 21 & 1. $4059 \mathrm{E}-18$ & $1.8798 \mathrm{E}-18$ & $2.3596 \mathrm{E}-18$ & $4.1821 \mathrm{E}-18$ & 8. $8513 \mathrm{E}-18$ & $1.7225 E-\odot 6$ & $2 \mathrm{E}-15$ & $9 \mathrm{E}-17$ & $4 E-\odot 9$ & $9 E-18$ \\
\hline 22 & OE-20 & E-19 & $3 E-19$ & E-19 & $3 E-19$ & $\odot E-\odot 9$ & $2 E-16$ & $9 \mathrm{E}-18$ & E- $-\odot 9$ & $8 \mathrm{E}-20$ \\
\hline 23 & 8.0 & 1.0 & $\odot E-15$ & 2.0 & & 4 & & & $E-\odot 6$ & \\
\hline 24 & $\odot . \odot \odot \odot \odot E+\odot \odot$ & $\odot . \odot \odot \odot \odot E+\odot \odot$ & $\odot . \odot \odot \odot \odot E+\odot \odot$ & $\odot .0 \odot \odot \odot \mathrm{E}+\odot \odot$ & $\odot . \odot \odot \odot \odot E+\odot \odot$ & $\odot . \odot \odot \odot \odot E+\odot \odot$ & $\odot E+\odot \odot$ & $\odot . \odot \odot \odot \odot E+\odot \odot$ & $\odot \mathrm{E}+\odot \odot$ & $\ominus \odot E+\odot \odot$ \\
\hline 25 & $2.9629 E-\odot 6$ & $3.4502 \mathrm{E}-16$ & $7.8861 \mathrm{E}-17$ & $2.6560 \mathrm{E}-17$ & $7 E-17$ & $6 \mathrm{E}-18$ & $4 \mathrm{E}-18$ & $3.3368 \mathrm{E}-18$ & OE -18 & $8 \mathrm{E}-11$ \\
\hline r & 1.3 & $\mathrm{E}-12$ & 1.2 & $7 \mathrm{E}-17$ & & & & & & \\
\hline & $7.0805 \mathrm{E}-06$ & 3. $9929 \mathrm{E}-07$ & $2.9198 \mathrm{E}-08$ & $1.4144 \mathrm{E}-07$ & $9.1390 \mathrm{E}-08$ & $5 E-06$ & $10 \mathrm{E}-04$ & $2.2502 \mathrm{E}-\odot 8$ & $34 \mathrm{E}-06$ & $7.9741 \mathrm{E}-06$ \\
\hline
\end{tabular}


Table 6 (continued). Dose rates $(\mathrm{rem} / \mathrm{h})$ for SRS Rad waste container case A (10.16 cm lead)

\begin{tabular}{|c|c|c|c|c|c|c|c|c|}
\hline rce & 11 & 12 & det & $\operatorname{det}$ & 15 & det & 17 & de \\
\hline 1 & $2755 E-\odot 6$ & $3337 E-06$ & $6031 \mathrm{E}-06$ & $3396 \mathrm{E}-07$ & $2.5725 \mathrm{E}-15$ & $4.2810 \mathrm{E}-16$ & 1. $6956 \mathrm{E}-16$ & $1865 \mathrm{E}$ \\
\hline 2 & $5.2785 E-08$ & . $1011 \mathrm{E}-12$ & 1. $3715 \mathrm{E}-\odot 9$ & $3.1015 \mathrm{E}-07$ & $1.7966 \mathrm{E}-11$ & $3.3862 \mathrm{E}-15$ & $1.1910 \mathrm{E}-17$ & $3.9901 \mathrm{E}-07$ \\
\hline 3 & $3.0158 \mathrm{E}-14$ & $.8060 \mathrm{E}-14$ & 2. $0805 \mathrm{E}-17$ & $6.0352 \mathrm{E}-12$ & $6.8997 \mathrm{E}-13$ & $1.8536 \mathrm{E}-18$ & $3.6238 \mathrm{E}-19$ & $9.2923 \mathrm{E}-12$ \\
\hline 4 & 1. $0860 \mathrm{E}-15$ & $178 E-14$ & $1.8700 \mathrm{E}-18$ & 1. $4448 \mathrm{E}-14$ & $7.1590 \mathrm{E}-10$ & $1.4530 \mathrm{E}-17$ & $4.3086 \mathrm{E}-19$ & $.9404 \mathrm{E}-12$ \\
\hline 5 & $68 \mathrm{E}-17$ & $0 E-14$ & $8 E-18$ & $1.5761 \mathrm{E}-16$ & L3E- - 8 & 1. $0317 \mathrm{E}-15$ & & \\
\hline 6 & $8.2916 \mathrm{E}-18$ & $2.4286 \mathrm{E}-15$ & $8.2479 E-19$ & 4. $9199 \mathrm{E}-18$ & 6. $0819 \mathrm{E}-08$ & $5.7814 \mathrm{E}-13$ & $6.4095 \mathrm{E}-19$ & $2.2701 \mathrm{E}-14$ \\
\hline 7 & $2.7970 \mathrm{E}-18$ & $1.5279 \mathrm{E}-15$ & $6.5711 \mathrm{E}-19$ & 1. $3026 \mathrm{E}-18$ & $6.7140 \mathrm{E}-10$ & $6.2403 \mathrm{E}-10$ & $8.0771 \mathrm{E}-19$ & 184E-15 \\
\hline 8 & $1.1661 \mathrm{E}-18$ & $9 \mathrm{E}-16$ & $5 E-19$ & $2 \mathrm{E}-19$ & $3 E-13$ & $56 E-08$ & $4 E-18$ & $3 E-17$ \\
\hline 9 & 3. $3177 \mathrm{E}-19$ & E- 15 & $34 \mathrm{E}-19$ & $3.6847 \mathrm{E}-19$ & $3 \mathrm{E}-17$ & $3.5788 \mathrm{E}-\odot 8$ & 38E-19 & $\mathrm{E}-17$ \\
\hline 10 & $2.6619 \mathrm{E}-19$ & $5.2408 \mathrm{E}-16$ & $2.0304 \mathrm{E}-19$ & $3.2883 \mathrm{E}-19$ & $4.4111 \mathrm{E}-18$ & 1. 4158E- $\odot 9$ & $8.7139 \mathrm{E}-19$ & LE-18 \\
\hline 11 & $2.3056 \mathrm{E}-19$ & $6 \mathrm{E}-16$ & OE-19 & $9 \mathrm{E}-19$ & $3 E-18$ & $2.3951 \mathrm{E}-11$ & $1 \mathrm{E}-18$ & $8 E-18$ \\
\hline 12 & $\mathrm{E}-19$ & $E-16$ & $7 E-19$ & $E-19$ & $E-19$ & $8 E-13$ & OE-18 & $4 \mathrm{E}-18$ \\
\hline 13 & $1.8839 \mathrm{E}-19$ & $E-16$ & $8 E-19$ & $1 E-19$ & $2 E-19$ & $7.1802 \mathrm{E}-15$ & $1 \mathrm{E}-18$ & -19 \\
\hline 14 & E-19 & $\mathrm{HE}-16$ & $9 E-19$ & $8 \mathrm{E}-19$ & $1 E-19$ & $2.0271 \mathrm{E}-16$ & $4 \mathrm{E}-17$ & OE-19 \\
\hline 15 & EE-19 & $E-16$ & $E-19$ & E-19 & $9 \mathrm{E}-19$ & $2 \mathrm{E}-17$ & $5 E-15$ & $2 E-19$ \\
\hline 16 & $1.4574 \mathrm{E}-19$ & $6 E-16$ & 1. $2759 \mathrm{E}-19$ & $1.8479 \mathrm{E}-19$ & $4.9838 \mathrm{E}-19$ & $2.8976 \mathrm{E}-18$ & $37 E-14$ & $52 E-19$ \\
\hline 17 & $\mathrm{E}-19$ & $E-16$ & E-19 & $E-19$ & $1 E-19$ & $1 E-18$ & $3 E-12$ & $2 \mathrm{E}-19$ \\
\hline 18 & $7 E-19$ & $E-16$ & E-19 & E-19 & E-19 & $3 E-19$ & $7 \mathrm{E}-10$ & $5 E-19$ \\
\hline 19 & $1.1827 \mathrm{E}-19$ & $9.4868 \mathrm{E}-17$ & 1. $0464 \mathrm{E}-19$ & $9 E-19$ & $6 E-19$ & $34 \mathrm{E}-19$ & $7 \mathrm{E}-\odot 9$ & $37 \mathrm{E}-19$ \\
\hline 20 & 1. $6625 \mathrm{E}-18$ & $9 \mathrm{E}-1$ & $1.4752 \mathrm{E}-18$ & $2 \mathrm{E}-18$ & S1E-18 & 1. $0098 \mathrm{E}-17$ & $9 \mathrm{E}-07$ & $29 E-18$ \\
\hline 21 & 1.5 & 2.4 & 1.3 & $E-18$ & EE-18 & $4 \mathrm{E}-18$ & 4. & $6 \mathrm{E}-18$ \\
\hline 22 & $2 E-20$ & $\mathrm{E}-15$ & $3 E-20$ & E-19 & $E-19$ & $1 E-19$ & $4 E-\odot 9$ & $8 E-19$ \\
\hline 23 & $9.0527 \mathrm{E}-16$ & $3.0491 \mathrm{E}-15$ & $7.8314 \mathrm{E}-16$ & 1. $0194 \mathrm{E}-15$ & $2.0985 \mathrm{E}-15$ & $8.2566 \mathrm{E}-14$ & 1.2967E-06 & 1. $0532 \mathrm{E}-15$ \\
\hline 24 & $\odot . \odot \odot \odot \odot E+\odot \odot$ & $\Theta \mathrm{E}+\Theta \odot$ & $\Theta E+\Theta \odot$ & $\ominus \mathrm{E}+\Theta \odot$ & $\Theta E+\Theta \odot$ & $\odot . \odot \odot \odot \odot E+\odot \odot$ & $\odot \mathrm{E}+\odot \odot$ & $\odot .0 \odot \odot \odot E+\odot \odot$ \\
\hline 25 & $6 \mathrm{E}-12$ & $\mathrm{~F}-14$ & $4 E-10$ & $4 E-09$ & $3 E-17$ & $5 \mathrm{E}-17$ & $5 E-18$ & $49 \mathrm{E}-12$ \\
\hline 26 & 1. $0796 \mathrm{E}-07$ & 1. $2905 \mathrm{E}-12$ & 1.1905E-10 & 1. $9957 \mathrm{E}-\odot 8$ & $2.7548 \mathrm{E}-16$ & 1. $4953 \mathrm{E}-17$ & $5.6762 \mathrm{E}-18$ & $2.6373 \mathrm{E}-11$ \\
\hline total & $1.4362 \mathrm{E}-06$ & 7.8337E-06 & $6.6048 \mathrm{E}-06$ & $5.6631 \mathrm{E}-07$ & 1. $2324 \mathrm{E}-07$ & $9.8309 \mathrm{E}-08$ & $2.3204 \mathrm{E}-06$ & $4.0024 \mathrm{E}-07$ \\
\hline
\end{tabular}


Table 7. Dose rates $(\mathrm{rem} / \mathrm{h})$ for SRS Rad waste container Case B (no lead)

\begin{tabular}{|c|c|c|c|c|c|c|c|c|c|c|}
\hline ce & 1 & et & det. & 4 & 5 & $\operatorname{det}$ & det & det & det & de \\
\hline 1 & $\odot \mathrm{E}+01$ & $31 \mathrm{E}+00$ & $848 \mathrm{E}+0 \odot$ & $319 \mathrm{E}+\odot \odot$ & 82E-01 & 74E- 01 & $14 \mathrm{E}$ & $28 \mathrm{E}-01$ & $28 E-08$ & $85 \mathrm{E}$ \\
\hline 2 & $8.6410 \mathrm{E}+0 \odot$ & $7.9350 \mathrm{E}+01$ & $1.6158 \mathrm{E}+01$ & $6.8306 \mathrm{E}+00$ & $38 \mathrm{E}+00$ & $9.2484 \mathrm{E}-01$ & $99 E-01$ & $94 \mathrm{E}-01$ & $921 E-\odot 8$ & $56 \mathrm{E}+$ \\
\hline 3 & $3.4596 \mathrm{E}-02$ & $1.1695 \mathrm{E}-01$ & $5.3614 \mathrm{E}-01$ & $1.1449 \mathrm{E}-01$ & 1. 4095E-๑2 & $1.1368 \mathrm{E}-03$ & . 3680E-๑3 & $2.6343 \mathrm{E}-03$ & $2.3429 E-\odot 9$ & $7.5783 \mathrm{E}-03$ \\
\hline 4 & $74 \mathrm{E}-02$ & $4 \mathrm{E}-02$ & $1 F-01$ & $36 \mathrm{E}-01$ & $73 \mathrm{E}-02$ & $2 \mathrm{E}-03$ & $62 \mathrm{E}-03$ & 78E- -3 & $5 E-\odot 9$ & \\
\hline 5 & $1.6963 \mathrm{E}-02$ & $3.9915 \mathrm{E}-02$ & $1 \mathrm{E}-02$ & $6.4436 \mathrm{E}-01$ & 35E-02 & $2.1248 \mathrm{E}-\odot 3$ & $6 \mathrm{E}-03$ & $5 E-\odot 3$ & $6 \mathrm{E}-\odot 8$ & \\
\hline 6 & 1. 2083E- -2 & $2.6320 \mathrm{E}-02$ & 4.1351E-02 & $6.3794 \mathrm{E}-01$ & 1. 1203E-01 & $3.0809 E-03$ & $5.9366 \mathrm{E}-03$ & 4. 2887E- 03 & 4. 9918E-๑8 & 1. $29 \odot 4 \mathrm{E}-03$ \\
\hline 7 & 8. 5559E-०3 & 1. 8651E- 02 & 2. $0875 E-02$ & $5 E-01$ & $2.4868 \mathrm{E}-01$ & $347 E-03$ & $92 \mathrm{E}-03$ & 386E- - & $5 E-\odot 7$ & $73 E-04$ \\
\hline 8 & E-०3 & $=-02$ & $E-\odot 2$ & $\mathrm{E}-01$ & $3 E-01$ & $9 \mathrm{E}-02$ & $9 \mathrm{E}-03$ & $3 E-03$ & & \\
\hline 9 & $1.8986 \mathrm{E}-03$ & E- 03 & E- 03 & $\mathrm{E}-02$ & $\mathrm{E}-01$ & 9E-०3 & $7 \mathrm{E}-03$ & $4 \mathrm{E}-03$ & & \\
\hline 10 & $9.9544 \mathrm{E}-04$ & $\mathrm{E}-03$ & $\mathrm{E}-03$ & $3 E-02$ & $2 \mathrm{E}-01$ & 1. $0711 \mathrm{E}-02$ & $7 E-03$ & 5E- - & $8 \mathrm{E}-06$ & $2 E-04$ \\
\hline 11 & $\mathrm{E}-\odot 3$ & $E-\odot 3$ & $-\odot 3$ & $\mathrm{E}-02$ & $4 \mathrm{E}-01$ & $\mathrm{E}-02$ & -03 & & & $5 \cap 0$ \\
\hline 12 & E-03 & $E-03$ & -03 & $\mathrm{E}-02$ & E- -2 & $\mathrm{E}-02$ & $4 \mathrm{E}-03$ & 03 & 1. & 4 \\
\hline 13 & E-९3 & E- 03 & -03 & E- -3 & $5 E-02$ & $5 E-02$ & $E-03$ & E-०3 & 04 & E- - 4 \\
\hline 14 & $=-03$ & -03 & -03 & $=-03$ & $E-02$ & $E-02$ & $E-\odot 2$ & उE-๑3 & & E-๑4 \\
\hline 15 & $9.2634 \mathrm{E}-\odot 4$ & E- -03 & $\mathrm{E}-03$ & $5 E-03$ & $31 E-\odot 2$ & $6 \mathrm{E}-02$ & $4 E-02$ & $9 \mathrm{E}-03$ & $E-03$ & $6 \mathrm{E}-\odot 4$ \\
\hline 16 & $8.2252 E-\odot 4$ & BE- 03 & E-03 & $8 E-\odot 3$ & $3 E-\odot 2$ & $6 \mathrm{E}-02$ & $\mathrm{E}-\mathrm{O} 2$ & $3 E-03$ & 03 & $3 E-\odot 4$ \\
\hline 17 & -04 & 1.6 & -03 & 3 & 92 & $\mathrm{E}-01$ & 02 & & & E- -4 \\
\hline 18 & $3 E-\odot 4$ & $E-03$ & $\mathrm{E}-03$ & $3 E-03$ & $5 E-\odot 2$ & $9 \mathrm{E}-01$ & $4 E-02$ & $\odot 2$ & 1.2 & $9 E-\odot 4$ \\
\hline 19 & $5.9873 E-\odot 4$ & $4 E-03$ & $8.6926 \mathrm{E}-04$ & $7 E-\odot 3$ & $9.3027 \mathrm{E}-\odot 3$ & $2.7452 \mathrm{E}-01$ & $4 \mathrm{E}-\odot 2$ & $1 \mathrm{E}-02$ & $3 E-\odot 3$ & $9 E-\odot 4$ \\
\hline 20 & -03 & 1.8 & & & -01 & $3 E+\odot \odot$ & $E-01$ & & & \\
\hline 21 & $=-03$ & 1.6 & 9.8 & 2 & E- 02 & $E+\odot \odot$ & 1 & & & $\mathrm{~F}$ \\
\hline 22 & 4. 4๑2ОE-๑4 & $9.8635 \mathrm{E}-04$ & $5.7209 E-04$ & 3E- - 3 & 4. 4377E- 03 & $2.7687 \mathrm{E}-01$ & $7 \mathrm{E}-02$ & $4 \mathrm{E}-02$ & $27 E-03$ & $33 E-04$ \\
\hline 23 & $E+\odot \odot$ & $+\odot \odot$ & $+\odot \odot$ & +01 & $3 E+01$ & $\mathrm{E}+02$ & & & & \\
\hline 24 & & 7.7 & -03 & & & & & & $\odot \odot$ & \\
\hline 25 & $1.6367 \mathrm{E}+01$ & 1. $\odot 803 \mathrm{E}+\odot \odot$ & E-01 & 4. 4231E-02 & 1. 3277E- 02 & $3.0960 \mathrm{E}-03$ & $9 \mathrm{E}-03$ & $5 E-03$ & $17 \mathrm{E}-14$ & SOE-๑ \\
\hline 26 & 1. $0408 \mathrm{E}+01$ & $4.0066 \mathrm{E}+0 \odot$ & $7.0645 E-01$ & $8 E-02$ & $2.3752 \mathrm{E}-\mathrm{-} 2$ & 8. $0731 \mathrm{E}-03$ & $3.0741 \mathrm{E}-03$ & $2.1761 \mathrm{E}-03$ & $3 E-12$ & 6. \\
\hline & $6.0914 \mathrm{E}+01$ & $9.20 \odot 2 \mathrm{E}+\odot 1$ & $2.9253 \mathrm{E}+01$ & $2.0316 \mathrm{E}+01$ & $2.3792 \mathrm{E}+01$ & $1.4007 \mathrm{E}+\odot 2$ & $7.4763 \mathrm{E}+02$ & $1.3498 \mathrm{E}+02$ & $1.5802 \mathrm{E}+01$ & $9.3142 \mathrm{E}+\odot$ \\
\hline
\end{tabular}


Table 7 (continued). Dose rates ( $\mathrm{rem} / \mathrm{h}$ ) for SRS Rad waste container Case B (no lead)

\begin{tabular}{|c|c|c|c|c|c|c|c|c|}
\hline Source & det. & $\operatorname{det}$ & $\operatorname{det}$ & $\operatorname{det}$ & det. & $\operatorname{det}$ & $\operatorname{det}$ & $\operatorname{det}$ \\
\hline 1 & $3.9740 \mathrm{E}+01$ & $9.0563 E+01$ & $3249 E+01$ & $1.4516 \mathrm{E}+01$ & $1.6817 \mathrm{E}+0 \odot$ & $7.0360 \mathrm{E}-01$ & 3. $0933 \mathrm{E}-01$ & $1541 \mathrm{E}+0 \odot$ \\
\hline 2 & $3.3287 \mathrm{E}+01$ & $4.8930 \mathrm{E}-\odot 3$ & $5.1650 \mathrm{E}+\odot \odot$ & $2.1524 \mathrm{E}+01$ & $2.5149 \mathrm{E}+\odot \odot$ & 1. $\odot 754 \mathrm{E}+\odot \odot$ & 553E- - 1 & $92 \mathrm{E}+01$ \\
\hline 3 & $6.4729 \mathrm{E}-04$ & $7.8280 E-\odot 6$ & $3.5137 \mathrm{E}-02$ & $1.1110 \mathrm{E}-01$ & $4.5785 E-02$ & $6.3285 \mathrm{E}-03$ & $2.2848 E-05$ & $4.7468 \mathrm{E}-03$ \\
\hline 4 & $4.2014 \mathrm{E}-\odot 4$ & 6. 6192E-०6 & . 2990E-02 & $6.1473 \mathrm{E}-02$ & 1. 2121E-01 & 8. 8183E-०3 & 1. $0100 \mathrm{E}-04$ & 1. $8012 \mathrm{E}-\mathrm{-} 2$ \\
\hline 5 & 1. 5115E-๑4 & 36E- - & $42 E-02$ & 329E- 02 & $2.2815 \mathrm{E}-01$ & $8 E-02$ & $2 E-05$ & $E-02$ \\
\hline 6 & $2.2192 \mathrm{E}-\odot 4$ & $2.7062 \mathrm{E}-07$ & $1.1252 \mathrm{E}-02$ & $2.2310 \mathrm{E}-02$ & $2.3061 \mathrm{E}-01$ & $4.5110 \mathrm{E}-02$ & $1.8780 \mathrm{E}-\odot 4$ & $2.5011 \mathrm{E}-02$ \\
\hline 7 & $4.3068 \mathrm{E}-04$ & $1.2139 \mathrm{E}-07$ & $.6484 \mathrm{E}-03$ & $1.5047 \mathrm{E}-02$ & 1. $2075 \mathrm{E}-01$ & $1.2022 \mathrm{E}-01$ & $68 \mathrm{E}-04$ & $35 E-02$ \\
\hline 8 & $6 E-\odot 4$ & ๑E-๑8 & $73 E-03$ & $8 \mathrm{E}-03$ & $57 E-02$ & $5 E-01$ & $5 E-\odot 4$ & \\
\hline 9 & $8.6860 \mathrm{E}-\odot 4$ & 4. 4987E-๑7 & 957E- - 3 & & $1.2146 \mathrm{E}-02$ & $5 E-01$ & $6 E-\odot 4$ & $1 E-03$ \\
\hline 10 & $9.1539 \mathrm{E}-04$ & 1. $0337 \mathrm{E}-07$ & $2.2132 \mathrm{E}-\odot 3$ & $2.4609 \mathrm{E}-03$ & $9.0158 \mathrm{E}-03$ & $9.1913 E-02$ & $9 E-\odot 4$ & $5 E-03$ \\
\hline 11 & $7 E-03$ & E-๑8 & $E-\odot 3$ & $5 E-03$ & $\mathrm{E}-\odot 3$ & $9 E-02$ & ๑E-๑3 & $2 \mathrm{E}-03$ \\
\hline 12 & 1. $2805 \mathrm{E}-03$ & $7 E-\odot 8$ & $6 \mathrm{E}-\odot 3$ & $8 E-03$ & $6 \mathrm{E}-\odot 3$ & $3 E-\odot 2$ & $7 E-\odot 3$ & $3 E-03$ \\
\hline 13 & $2.4591 \mathrm{E}-03$ & $2.6750 \mathrm{E}-\odot 8$ & $1.5723 \mathrm{E}-\odot 3$ & $4 E-03$ & $5 E-\odot 3$ & $3 E-02$ & $4 \mathrm{E}-\odot 3$ & $9 \mathrm{E}-03$ \\
\hline 14 & E-03 & E-०8 & E- -3 & $2 E-\odot 3$ & $1 E-\odot 4$ & $2 E-\odot 3$ & $2 E-\odot 3$ & $6 \mathrm{E}-03$ \\
\hline 15 & $2.1809 \mathrm{E}-03$ & बE-๑8 & $8 E-\odot 3$ & ๑E- ๑3 & $9 E-\odot 4$ & $1 E-03$ & $4 E-\odot 2$ & $2 E-03$ \\
\hline 16 & 1.9887E- 03 & $7 E-\odot 8$ & 1.1753E-๑3 & $\odot E-\odot 3$ & $7.8321 \mathrm{E}-05$ & $\odot E-\odot 3$ & $5 E-\odot 2$ & $97 E-03$ \\
\hline 17 & $E-03$ & E-๑8 & $5 E-03$ & $2 E-\odot 4$ & $3 E-05$ & H- 03 & $5 E-\odot 2$ & $2 \mathrm{E}-03$ \\
\hline 18 & $9 E-03$ & $1 E-\odot 9$ & $7 E-\odot 4$ & $4 E-\odot 4$ & BE-05 & $5 E-03$ & $4 E-\odot 2$ & $2 \mathrm{E}-03$ \\
\hline 19 & 1. 5397E- 03 & $7.6911 \mathrm{E}-\odot 9$ & $8.7790 \mathrm{E}-\odot 4$ & $7 E-04$ & $2.4 \odot 29 E-05$ & $1 E-04$ & $9 E-01$ & $7 E-03$ \\
\hline 20 & $2.11 \mathrm{~s}$ & E- - 7 & $1 E-\odot 2$ & $3 E-03$ & $2 E-\odot 4$ & $5-03$ & $6 \mathrm{E}+\odot \odot$ & $2 \mathrm{E}-02$ \\
\hline 21 & E-०2 & $E-07$ & $L E-\odot 2$ & $\mathrm{E}-03$ & 1.3 & פE- 03 & $8 \mathrm{E}+\odot \odot$ & $6 \mathrm{E}-02$ \\
\hline 22 & 1.1914E- 03 & 8.1652E-๑8 & $6.4335 \mathrm{E}-\odot 4$ & $7 E-04$ & $4.4706 \mathrm{E}-06$ & $65 E-04$ & $1 \mathrm{E}-01$ & $30 E-03$ \\
\hline 23 & $E+\odot \odot$ & כE- $\odot 7$ & $4 \mathrm{E}+\odot \odot$ & $5 \mathrm{E}+00$ & $3 \mathrm{E}+\odot \odot$ & $7 \mathrm{E}+\odot \odot$ & $6 \mathrm{E}+01$ & $2 \mathrm{E}+00$ \\
\hline 24 & 1.86 & 2.0 & $8 F-04$ & $7 E-\odot 4$ & $9 E-04$ & $2 E-02$ & $5 E-\odot 1$ & $8 \mathrm{E}-03$ \\
\hline 25 & $3.3051 E-04$ & $3.2076 \mathrm{E}-\odot 1$ & $9.7745 \mathrm{E}-\odot 2$ & $9 \mathrm{E}-01$ & $2.0854 \mathrm{E}-02$ & $5 E-\odot 3$ & $3 E-\odot 6$ & $34 \mathrm{E}-01$ \\
\hline 26 & $2.0562 \mathrm{E}-01$ & $9 \mathrm{E}-02$ & $3 E-02$ & $5 E-01$ & $2.4108 \mathrm{E}-02$ & $3.5882 \mathrm{E}-03$ & $9 E-\odot 6$ & $6 \mathrm{E}-01$ \\
\hline & $7.7547 \mathrm{E}+01$ & $9.0905 \mathrm{E}+01$ & $4.2688 E+01$ & $4.2923 E+01$ & $7.7 \odot 93 \mathrm{E}+\odot \odot$ & $1.2563 \mathrm{E}+01$ & $6.6153 \mathrm{E}+01$ & 1. $0698 \mathrm{E}+\odot 2$ \\
\hline
\end{tabular}


Table 8. Dose rates $(\mathrm{rem} / \mathrm{h})$ for SRS Rad waste container Case B $(5.08 \mathrm{~cm}$ lead)

\begin{tabular}{|c|c|c|c|c|c|c|c|c|c|c|}
\hline e & 1 & 2 & 3 & 4 & 5 & 6 & 7 & det & det & \\
\hline 1 & $6.3438 \mathrm{E}-03$ & $7 E-06$ & $70 E-05$ & 9557E- - & 7E-11 & $88 E-15$ & $58 \mathrm{E}-$ & $402 \mathrm{E}-16$ & $56 \mathrm{E}-13$ & \\
\hline 2 & $2.3264 \mathrm{E}-05$ & $82 \mathrm{E}-02$ & $36 \odot E-\odot 4$ & 7826E- - 66 & $544 E-08$ & $19 E-13$ & $61 \mathrm{E}-15$ & $447 \mathrm{E}-16$ & $52 \mathrm{E}-12$ & \\
\hline 3 & $2.5828 \mathrm{E}-14$ & $2.1057 \mathrm{E}-09$ & $9.8143 E-05$ & . 1976E- 09 & . $4780 \mathrm{E}-17$ & $5.4514 \mathrm{E}-19$ & $3.7978 \mathrm{E}-19$ & $.9232 \mathrm{E}-19$ & $.6962 \mathrm{E}-14$ & $4764 E-08$ \\
\hline 4 & $5 E-16$ & 7E-11 & $9 \mathrm{E}-07$ & $7 F-96$ & & 9 & & $1 E-19$ & & \\
\hline 5 & $3 E-17$ & $6 \mathrm{E}-14$ & $4 \mathrm{E}-10$ & $6 E-\odot 4$ & $4 \mathrm{E}-12$ & $4 E-19$ & & $9 \mathrm{E}-19$ & & \\
\hline 0 & $1.9462 \mathrm{E}-18$ & $79 E-16$ & $45 E-13$ & $2.1365 E-04$ & $03 E-09$ & $93 E-19$ & $9 \mathrm{E}$ & 19 & $5 E-12$ & te \\
\hline 1 & OE - 18 & $\mathrm{HE}-17$ & 4E - 15 & $8 E-06$ & $9 E-06$ & $4 \mathrm{E}-18$ & $6 \mathrm{E}-19$ & $4 E-19$ & 7E-12 & LEE \\
\hline 8 & 8.4 & $F-18$ & $F_{-17}$ & & & F-18 & & & & \\
\hline 9 & $\mathrm{E}-19$ & $E-19$ & E-18 & $E-12$ & & 17 & & & & \\
\hline 10 & 3.0 & $E-19$ & $\mathrm{LE}-19$ & -E - 14 & E- -05 & E - 17 & $1 E-19$ & $2 E-19$ & & \\
\hline 11 & 2.8 & $=-19$ & - & $E-15$ & $E-07$ & E- & $E-19$ & & & \\
\hline 12 & $E-19$ & $E-19$ & $E-19$ & -16 & $\mathrm{E}-\odot 8$ & E-15 & 1. & & & \\
\hline 13 & 2.4 & $E-19$ & $E-19$ & $E-17$ & 0 & $E-13$ & & & & \\
\hline 14 & 2.2 & $=-19$ & $E-19$ & -18 & $E-$ & 2 & $\mathrm{E}-17$ & 19 & & \\
\hline 15 & $E-19$ & $E-19$ & E-19 & -18 & $E-13$ & $\mathrm{E}-10$ & $1 E-16$ & & & 5 \\
\hline 16 & 1.9 & $E-19$ & E-19 & $E-19$ & $E-14$ & E-๑९ & $\mathrm{E}-16$ & 18 & $\Theta 7$ & $1 \mathrm{E}-17$ \\
\hline 17 & 1.7 & -1 & -19 & & 5 & 7 & 15 & & & \\
\hline 18 & 1.6 & $E-19$ & E-19 & $E-19$ & E-16 & E- 06 & $7 E-14$ & $E-17$ & & $4 \mathrm{E}-$ \\
\hline 19 & E-19 & $E-19$ & $E-19$ & $4 \mathrm{E}-19$ & $3 \mathrm{E}-17$ & OE- 05 & E-12 & $\mathrm{E}-16$ & $2 \mathrm{E}-\Theta 7$ & $3 \mathrm{E}-1$ \\
\hline 24 & 2.2 & $=18$ & $=18$ & & & & & & & \\
\hline 21 & 2.0 & 2.7 & E- & & 7 & 3 & 9 & & & ic \\
\hline 22 & $8 E-19$ & E-19 & OE - 19 & $2 \mathrm{E}-19$ & E-19 & E - 05 & $9 \mathrm{E}-\odot 9$ & $\mathrm{E}-14$ & 06 & $2 \mathrm{E}-1$ \\
\hline 23 & 8.0 & 1. & E-15 & & & & 2. & & & \\
\hline 2 & 2.2 & & & & & & & & & \\
\hline 25 & $4.8085 \mathrm{E}-03$ & $E-10$ & OE-15 & $2 \mathrm{E}-17$ & $2 \mathrm{E}-17$ & $\mathrm{E}$ & E-18 & $9 \mathrm{E}-18$ & 18 & $4 \mathrm{E}-\mathrm{C}$ \\
\hline 26 & $6.7286 \mathrm{E}-04$ & $E-06$ & $29 E-12$ & $9 \mathrm{E}-17$ & $\mathrm{E}-17$ & & $2 E-18$ & E-18 & $3 E-17$ & \\
\hline & $1.1848 \mathrm{E}-02$ & $1.4592 \mathrm{E}-\odot 2$ & $6.9496 \mathrm{E}-04$ & $4.5036 \mathrm{E}-04$ & $5 E-\odot 4$ & $29 E-03$ & $2.0478 \mathrm{E}-01$ & $7.5740 \mathrm{E}-\odot 3$ & $6.1131 E-03$ & 1. 4559E- \\
\hline
\end{tabular}


Table 8 (continued). Dose rates $(\mathrm{rem} / \mathrm{h})$ for SRS Rad waste container Case B $(5.08 \mathrm{~cm} l \mathrm{lead})$

\begin{tabular}{|c|c|c|c|c|c|c|c|c|}
\hline ource & det & det. & det & det. & det. & det & $\operatorname{det}$ & $\operatorname{det}$ \\
\hline 1 & $3.2698 E-03$ & 1. $3894 \mathrm{E}-02$ & $0377 \mathrm{E}-\odot 2$ & $4740 E-\odot 4$ & 1. $2900 \mathrm{E}-\odot 9$ & $2.9742 \mathrm{E}-13$ & 1. $8049 \mathrm{E}-16$ & $2.5653 \mathrm{E}-05$ \\
\hline 2 & $2.6555 \mathrm{E}-03$ & $.2506 \mathrm{E}-07$ & $9234 \mathrm{E}-04$ & $7562 \mathrm{E}-03$ & $1.2335 \mathrm{E}-05$ & $5.0451 \mathrm{E}-08$ & $1.8298 \mathrm{E}-12$ & 1. $4619 \mathrm{E}-02$ \\
\hline 3 & $1.7271 \mathrm{E}-09$ & $1.9581 \mathrm{E}-10$ & $3.6484 \mathrm{E}-11$ & $8.6420 \mathrm{E}-07$ & $1.3307 \mathrm{E}-07$ & $2.6229 \mathrm{E}-13$ & $5.4146 \mathrm{E}-19$ & $9.2662 \mathrm{E}-08$ \\
\hline 4 & $2.2058 \mathrm{E}-10$ & $1.9026 \mathrm{E}-10$ & 7. $0820 E-13$ & $1.9440 \mathrm{E}-\odot 8$ & $8.5200 \mathrm{E}-06$ & $1.2773 \mathrm{E}-11$ & $6.6878 \mathrm{E}-19$ & $2.0407 \mathrm{E}-07$ \\
\hline 5 & $1.1590 \mathrm{E}-11$ & $1.6461 \mathrm{E}-10$ & $1.7586 \mathrm{E}-14$ & 3. $0974 \mathrm{E}-10$ & $1.2037 \mathrm{E}-04$ & $1.1221 \mathrm{E}-09$ & 8.8512E-19 & $2.0827 \mathrm{E}-07$ \\
\hline 6 & $2.9349 \mathrm{E}-12$ & $6.7005 \mathrm{E}-12$ & $8.9687 \mathrm{E}-16$ & $5.1633 \mathrm{E}-12$ & $1.2132 \mathrm{E}-04$ & 1. $2019 \mathrm{E}-07$ & $2.6765 \mathrm{E}-18$ & $2.3367 \mathrm{E}-08$ \\
\hline 7 & 1. $0901 \mathrm{E}-12$ & $2.8012 \mathrm{E}-12$ & $8.7311 \mathrm{E}-17$ & $9.2208 \mathrm{E}-14$ & $8.2392 \mathrm{E}-06$ & $7.9297 \mathrm{E}-06$ & 1. $0101 \mathrm{E}-17$ & $2.3347 \mathrm{E}-09$ \\
\hline 8 & $3.6074 \mathrm{E}-13$ & $1.6517 \mathrm{E}-12$ & $1.1329 \mathrm{E}-17$ & $2.6248 \mathrm{E}-15$ & $1.2687 \mathrm{E}-07$ & $1.2217 \mathrm{E}-04$ & $8.3865 \mathrm{E}-17$ & $2.2547 \mathrm{E}-10$ \\
\hline 9 & $6.5493 \mathrm{E}-14$ & $1.5282 \mathrm{E}-11$ & $4 E-19$ & $7 \mathrm{E}-18$ & $8 E-11$ & $8.1122 \mathrm{E}-05$ & $6 E-16$ & $3 E-11$ \\
\hline 10 & $2.7540 \mathrm{E}-14$ & $3.2220 \mathrm{E}-12$ & $3.0431 E-19$ & $9.4182 \mathrm{E}-19$ & $2.4869 \mathrm{E}-12$ & $9.8728 E-\odot 6$ & $6.0542 \mathrm{E}-16$ & 1. $9162 \mathrm{E}-11$ \\
\hline 11 & $1.4786 \mathrm{E}-14$ & 1. $0194 \mathrm{E}-12$ & $2.7827 \mathrm{E}-19$ & $5.6053 \mathrm{E}-19$ & 1. $0527 \mathrm{E}-13$ & $7.8945 E-\odot 7$ & $1.1132 \mathrm{E}-14$ & $94 \mathrm{E}-12$ \\
\hline 12 & $8.6698 \mathrm{E}-15$ & $8.2073 E-13$ & $2.5601 \mathrm{E}-19$ & $5 \mathrm{E}-19$ & $8 E-15$ & 1. $0171 \mathrm{E}-07$ & $46 E-13$ & $36 \mathrm{E}-12$ \\
\hline 13 & $9.4666 \mathrm{E}-15$ & $6.6023 E-13$ & $2.3647 \mathrm{E}-19$ & $3.7060 \mathrm{E}-19$ & $4.6290 \mathrm{E}-16$ & $5.2727 \mathrm{E}-\odot 9$ & $2.3523 \mathrm{E}-12$ & 7. $0179 \mathrm{E}-13$ \\
\hline 14 & $3.8200 \mathrm{E}-15$ & $5.3458 E-13$ & $1 E-19$ & $8 E-19$ & $3 \mathrm{E}-17$ & $9 E-10$ & $79 E-11$ & $4 E-13$ \\
\hline 15 & $1.4612 \mathrm{E}-15$ & $4.2842 \mathrm{E}-13$ & $9 \mathrm{E}-19$ & $1 \mathrm{E}-19$ & $6 \mathrm{E}-18$ & $30 E-11$ & $\odot \mathrm{E}-\odot 9$ & $5 E-14$ \\
\hline 16 & $5.9856 \mathrm{E}-16$ & $3.4847 \mathrm{E}-13$ & $1.8965 \mathrm{E}-19$ & $2.7541 \mathrm{E}-19$ & $1.7862 \mathrm{E}-18$ & $8.1837 \mathrm{E}-13$ & $1.5258 \mathrm{E}-\odot 8$ & $2.8582 \mathrm{E}-14$ \\
\hline 17 & $2.6294 \mathrm{E}-16$ & $2.8114 \mathrm{E}-13$ & $1.7707 \mathrm{E}-19$ & $2.5345 \mathrm{E}-19$ & $7.7657 \mathrm{E}-19$ & $2.4058 \mathrm{E}-14$ & $3.2393 E-07$ & 1. $0284 \mathrm{E}-14$ \\
\hline 18 & 1. $2315 \mathrm{E}-16$ & $2.2721 \mathrm{E}-13$ & OE-19 & $8 \mathrm{E}-19$ & $E-19$ & $1 E-15$ & $7 E-06$ & L3E-15 \\
\hline 19 & $6.0667 \mathrm{E}-17$ & $1.8201 \mathrm{E}-13$ & $7 \mathrm{E}-19$ & $6 \mathrm{E}-19$ & $9 \mathrm{E}-19$ & $1 E-16$ & $6 \odot E-\odot 5$ & $39 E-15$ \\
\hline 20 & $4.6385 \mathrm{E}-16$ & $2.4350 \mathrm{E}-12$ & $2.1896 \mathrm{E}-18$ & 3. $0219 \mathrm{E}-18$ & $6.9009 \mathrm{E}-18$ & $4.6687 \mathrm{E}-16$ & $8.7192 \mathrm{E}-04$ & $9.9768 \mathrm{E}-15$ \\
\hline 21 & $2.4269 \mathrm{E}-16$ & 3. $0098 \mathrm{E}-11$ & $9 \mathrm{E}-18$ & $6 \mathrm{E}-18$ & $0 \mathrm{E}-18$ & $0 E-16$ & $1 E-04$ & $9 E-15$ \\
\hline 22 & LE-18 & $7 \mathrm{E}-12$ & $4 E-19$ & $3 E-19$ & $2 \mathrm{E}-19$ & $6 \mathrm{E}-18$ & $2 E-05$ & $0 \mathrm{E}-16$ \\
\hline 23 & $7.4207 \mathrm{E}-15$ & $2.5582 \mathrm{E}-12$ & $2.2081 \mathrm{E}-14$ & $3.4503 \mathrm{E}-13$ & $1.6687 \mathrm{E}-10$ & $1.135 \odot E-07$ & $5.6361 E-03$ & 1. $0420 \mathrm{E}-15$ \\
\hline 24 & $2.5662 \mathrm{E}-17$ & $4.1815 \mathrm{E}-17$ & $2.3130 \mathrm{E}-17$ & $3.5528 \mathrm{E}-17$ & $3.5140 \mathrm{E}-16$ & $2.7913 \mathrm{E}-13$ & $5.8944 \mathrm{E}-06$ & $2.9110 \mathrm{E}-17$ \\
\hline 25 & $6.8743 \mathrm{E}-09$ & $2.2745 \mathrm{E}-08$ & $2.0249 E-06$ & $2.4473 E-05$ & $0 \mathrm{E}-11$ & $9.2593 E-16$ & $5.0723 E-18$ & $4 E-06$ \\
\hline 26 & 1. $1941 \mathrm{E}-04$ & $3.6517 \mathrm{E}-08$ & $1.4372 \mathrm{E}-06$ & $9.0386 \mathrm{E}-05$ & 1. $2649 \mathrm{E}-10$ & $8.9294 \mathrm{E}-15$ & $5.6544 \mathrm{E}-18$ & $1.1808 \mathrm{E}-06$ \\
\hline tal & $6.0448 E-03$ & 1. $3894 \mathrm{E}-02$ & 1. $0573 \mathrm{E}-02$ & $9.7193 \mathrm{E}-\odot 3$ & $2.7105 \mathrm{E}-\odot 4$ & $2.2227 \mathrm{E}-\odot 4$ & 7. 4151E- 03 & 1. 4647E- -2 \\
\hline
\end{tabular}


Table 9. Dose rates $(\mathrm{rem} / \mathrm{h})$ for SRS Rad waste container Case B $(10.16 \mathrm{~cm}$ lead)

\begin{tabular}{|c|c|c|c|c|c|c|c|c|c|c|}
\hline rce & 1 & 2 & 3 & 4 & 5 & 6 & 7 & 8 & 9 & \\
\hline 1 & $E-06$ & 5.3 & 2.3 & 2.7 & $E-16$ & 6 & & & & \\
\hline 2 & $7286 \mathrm{E}-09$ & $7.9751 \mathrm{E}-06$ & 1. $6269 \mathrm{E}-07$ & 1. $3485 \mathrm{E}-10$ & $1.8448 \mathrm{E}-14$ & $2.4230 \mathrm{E}-16$ & $1.8304 \mathrm{E}-16$ & $1.4618 \mathrm{E}-16$ & $1.4183 \mathrm{E}-15$ & $1.4212 \mathrm{E}-\odot 9$ \\
\hline 3 & $353 E-18$ & $2342 \mathrm{E}-15$ & $2.7948 \mathrm{E}-\odot 8$ & $2.7670 \mathrm{E}-15$ & $2.4472 \mathrm{E}-18$ & $5.5096 \mathrm{E}-19$ & $3.8464 \mathrm{E}-19$ & $2.9658 \mathrm{E}-19$ & $.2852 \mathrm{E}-17$ & \\
\hline 4 & $2.4469 \mathrm{E}-18$ & 1. $3001 \mathrm{E}-17$ & $3.5218 \mathrm{E}-12$ & $2.9078 \mathrm{E}-11$ & $3.5939 \mathrm{E}-18$ & $6.5681 \mathrm{E}-19$ & $4.4462 \mathrm{E}-19$ & $3.3665 \mathrm{E}-19$ & $1.2376 \mathrm{E}-16$ & 897 \\
\hline 5 & $1.7764 \mathrm{E}-18$ & $3.7976 \mathrm{E}-18$ & $5.7550 \mathrm{E}-16$ & 1. $0939 \mathrm{E}-07$ & $7.6341 \mathrm{E}-18$ & 7. $9623 \mathrm{E}-19$ & $5.1966 \mathrm{E}-19$ & $3.8535 E-19$ & $3.7516 \mathrm{E}-16$ & 1.3790E-16 \\
\hline 6 & 1. $3457 \mathrm{E}-18$ & $2.5918 \mathrm{E}-18$ & $5.7234 \mathrm{E}-18$ & $.0163 \mathrm{E}-07$ & $2.2171 \mathrm{E}-15$ & $9.8498 \mathrm{E}-19$ & $6.1523 \mathrm{E}-19$ & $4.4533 E-19$ & $3 E-15$ & \\
\hline 7 & 1. $0536 \mathrm{E}-18$ & $1.8809 \mathrm{E}-18$ & $3.3304 \mathrm{E}-18$ & $2.5704 \mathrm{E}-11$ & $2.1372 \mathrm{E}-11$ & 1. $2493 \mathrm{E}-18$ & 7. $3941 \mathrm{E}-19$ & $5.2030 \mathrm{E}-19$ & $3 E-15$ & \\
\hline 8 & $8.4665 E-19$ & 1. $4217 \mathrm{E}-18$ & $2.2982 \mathrm{E}-18$ & $2.5176 \mathrm{E}-15$ & 8. $9050 \mathrm{E}-\odot 8$ & $1.6351 \mathrm{E}-18$ & $9.0470 \mathrm{E}-19$ & $6.1565 \mathrm{E}-19$ & $9.9582 \mathrm{E}-15$ & 1. $0815 \mathrm{E}-18$ \\
\hline 9 & $3.4258 \mathrm{E}-19$ & $5.4 \odot 44 \mathrm{E}-19$ & $8.2654 \mathrm{E}-19$ & 3. $6278 \mathrm{E}-18$ & $3.9120 \mathrm{E}-\odot 8$ & $1.1278 \mathrm{E}-18$ & $5.6096 \mathrm{E}-19$ & $3.6762 \mathrm{E}-19$ & $39 E-14$ & LE -19 \\
\hline 10 & $75 E-19$ & $5-19$ & OE-19 & $E-18$ & $7 \mathrm{E}-10$ & $7 \mathrm{E}-18$ & $2 \mathrm{E}-19$ & $3 E-19$ & & \\
\hline 11 & $2.8664 \mathrm{E}-19$ & 4. $3487 \mathrm{E}-19$ & $6.3086 \mathrm{E}-19$ & E-18 & 4. $0908 \mathrm{E}-12$ & LE-18 & $.724 \mathrm{E}$ & 19 & $5 E-12$ & 19 \\
\hline 12 & $2.6364 \mathrm{E}-19$ & $3.9315 \mathrm{E}-19$ & $5.5798 \mathrm{E}-19$ & $1.4470 \mathrm{E}-18$ & $2.6826 \mathrm{E}-14$ & 2. $0132 \mathrm{E}-18$ & $8.2004 \mathrm{E}-19$ & $4.9868 \mathrm{E}-19$ & $0 E-12$ & ๑०E-19 \\
\hline 13 & $2.4329 \mathrm{E}-19$ & $7 E-19$ & $E-19$ & $E-18$ & $7 E-16$ & $\mathrm{LE}-18$ & $2 \mathrm{E}-19$ & $6 E-19$ & $9 \mathrm{E}-11$ & $5 E-19$ \\
\hline 14 & $2.2520 \mathrm{E}-19$ & $3.2568 \mathrm{E}-19$ & 4. $4540 \mathrm{E}-19$ & 1. $0221 \mathrm{E}-18$ & $2.1417 \mathrm{E}-17$ & $6.9390 \mathrm{E}-18$ & $1.1004 \mathrm{E}-18$ & $6.2758 \mathrm{E}-19$ & $1 \mathrm{E}-10$ & $38 \mathrm{E}-19$ \\
\hline 15 & 2.0 & 2.98 & $3 E-19$ & $E-19$ & $E-18$ & E-16 & E-18 & $6 \mathrm{E}-19$ & $\mathrm{E}-10$ & $7 E-19$ \\
\hline 16 & $6 E-19$ & $3 E-19$ & $4 E-19$ & $5 E-19$ & $1 E-18$ & $8 E-15$ & $92 E-18$ & 77E-19 & $8 \mathrm{E}-10$ & $1 E-19$ \\
\hline 17 & $1.8153 \mathrm{E}-19$ & $2.5265 E-19$ & 3. $3093 \mathrm{E}-19$ & $6.6384 \mathrm{E}-19$ & 1. $7913 \mathrm{E}-18$ & $3.4851 \mathrm{E}-13$ & $70 E-18$ & $9.3215 \mathrm{E}-19$ & $9 \mathrm{E}-10$ & H1E-19 \\
\hline 18 & $E-19$ & $6 E-19$ & 3. $0243 \mathrm{E}-19$ & $E-19$ & $2 E-18$ & $\mathrm{E}-11$ & $\mathrm{E}-18$ & $9 \mathrm{E}-18$ & $E-10$ & $1 E-19$ \\
\hline 19 & $=-19$ & 2.1 & $E-19$ & $E-19$ & $E-18$ & E-๑९ & $E-18$ & $1 E-18$ & $E-10$ & คE-19 \\
\hline 20 & $2.2409 \mathrm{E}-18$ & $3.0228 \mathrm{E}-18$ & $3.8314 \mathrm{E}-18$ & $E-18$ & $1.5498 \mathrm{E}-17$ & $1 E-06$ & $36 E-16$ & $2.2434 \mathrm{E}-17$ & $2 E-\odot 9$ & $31 E-18$ \\
\hline 21 & $2.1083 \mathrm{E}-18$ & $2.8180 \mathrm{E}-18$ & $3.5389 \mathrm{E}-18$ & $6.2715 \mathrm{E}-18$ & 1. $3272 \mathrm{E}-17$ & $2.3490 E-\odot 6$ & $1.8404 \mathrm{E}-15$ & $2.6821 \mathrm{E}-17$ & 1. $2602 \mathrm{E}-\odot 8$ & $2.0438 \mathrm{E}-18$ \\
\hline 22 & 1.32 & 1.7 & $7 E-19$ & $E-19$ & 7.6 & $6 \mathrm{E}-\odot 9$ & $9 E-15$ & & $7 E-\odot 9$ & $5 E-19$ \\
\hline 23 & $8.0432 \mathrm{E}-16$ & 1.05 & 1. $2480 \mathrm{E}-15$ & 2.00 & 3.65 & E- $\odot 8$ & $\odot E-\odot 4$ & $2 E-\odot 8$ & $2 E-06$ & ๑E-16 \\
\hline 24 & $2.3109 \mathrm{E}-17$ & $2.9444 \mathrm{E}-17$ & $3.4237 \mathrm{E}-17$ & $5.1829 \mathrm{E}-17$ & $8.4123 \mathrm{E}-17$ & $9.6610 \mathrm{E}-15$ & $2.7393 \mathrm{E}-\odot 9$ & $4.6365 E-06$ & $3.2438 \mathrm{E}-06$ & $2.2917 \mathrm{E}-17$ \\
\hline 25 & $2.9629 \mathrm{E}-06$ & 3.45 & $7.8861 \mathrm{E}-17$ & $2.6560 \mathrm{E}-17$ & $\mathrm{E}-17$ & $6 \mathrm{E}-18$ & $4.0694 \mathrm{E}-18$ & $3.3368 E-18$ & OE-18 & $78 E-11$ \\
\hline 26 & 1. $3840 E-07$ & $2.3402 \mathrm{E}-12$ & 1. $2415 \mathrm{E}-16$ & $3.4177 \mathrm{E}-17$ & 1. $6004 \mathrm{E}-17$ & $5.8248 \mathrm{E}-18$ & $4.4853 \mathrm{E}-18$ & $3.6433 E-18$ & $2.9270 \mathrm{E}-18$ & $1.4373 \mathrm{E}-11$ \\
\hline total & $7.0850 E-06$ & $7.9756 \mathrm{E}-06$ & 1. $9299 \mathrm{E}-\odot 7$ & $2.1121 \mathrm{E}-07$ & $1.2888 \mathrm{E}-07$ & $4.7189 \mathrm{E}-06$ & $1.1811 \mathrm{E}-04$ & $4.6590 \mathrm{E}-06$ & $5.1930 \mathrm{E}-\odot 6$ & $7.9755 \mathrm{E}-\odot 6$ \\
\hline
\end{tabular}


Table 9 (continued). Dose rates $(\mathrm{rem} / \mathrm{h})$ for SRS Rad waste container Case B (10.16 cm lead)

\begin{tabular}{|c|c|c|c|c|c|c|c|c|}
\hline & 11 & 12 & 13 & 14 & 15 & & & \\
\hline 1 & $E-\odot 6$ & $E-\odot 6$ & $6.6031 \mathrm{E}-06$ & E- $-\odot 7$ & $5 E-15$ & $4.2810 E-16$ & $6956 \mathrm{E}-16$ & $5 E-\odot 9$ \\
\hline 2 & $1.0557 \mathrm{E}-06$ & $8.2022 \mathrm{E}-11$ & $2.7430 E-\odot 8$ & $6.2 \odot 29 E-\odot 6$ & $3.5123 \mathrm{E}-10$ & $6.7601 \mathrm{E}-14$ & $2.3820 \mathrm{E}-16$ & $7.9801 \mathrm{E}-06$ \\
\hline 3 & $5.1694 \mathrm{E}-14$ & $55 \mathrm{E}-13$ & 5702517 & & & $8864 \mathrm{E}-18$ & & \\
\hline 4 & $1.8817 \mathrm{E}-15$ & $1.1642 \mathrm{E}-13$ & $2.6615 \mathrm{E}-18$ & $524 \mathrm{E}-14$ & $3621 \mathrm{E}-10$ & $1.9150 \mathrm{E}-17$ & $6.4663 \mathrm{E}-19$ & -12 \\
\hline 5 & $6.1410 \mathrm{E}-17$ & 1. $0882 \mathrm{E}-13$ & $1.6059 \mathrm{E}-18$ & $1.9373 \mathrm{E}-16$ & $8.5836 E-\odot 8$ & 1. $2721 \mathrm{E}-15$ & $7.8127 \mathrm{E}-19$ & 1. $3980 \mathrm{E}-12$ \\
\hline 6 & $1.2763 \mathrm{E}-17$ & $348 E-15$ & 1. $2383 \mathrm{E}-18$ & $156 E-18$ & 3. 5498E- $\odot 8$ & 7. $0310 \mathrm{E}-13$ & $214 \mathrm{E}-19$ & $1 E-14$ \\
\hline 7 & 4. $3020 \mathrm{E}-18$ & $2.0020 \mathrm{E}-15$ & $9.8641 \mathrm{E}-19$ & $1.9344 \mathrm{E}-18$ & $8.7711 \mathrm{E}-10$ & $26 \mathrm{E}-10$ & $27 E-18$ & -15 \\
\hline 8 & $1.7789 \mathrm{E}-18$ & $1.2131 \mathrm{E}-15$ & $8.0277 \mathrm{E}-19$ & 1. $4088 \mathrm{E}-18$ & $7.7271 \mathrm{E}-13$ & 8. 4911E-๑8 & $1.5730 \mathrm{E}-18$ & 1. $3312 \mathrm{E}-16$ \\
\hline 9 & 4. $9731 \mathrm{E}-19$ & $1.1348 \mathrm{E}-14$ & $3.3302 \mathrm{E}-19$ & $5.5340 E-19$ & $07 \mathrm{E}-17$ & $18 \mathrm{E}-08$ & $35 \mathrm{E}-18$ & $67 \mathrm{E}-17$ \\
\hline 10 & $E-19$ & $4 \mathrm{E}-15$ & $7 E-19$ & ๑E-19 & $4 E-18$ & $1 E-\odot 9$ & $2 \mathrm{E}-18$ & \\
\hline 11 & $7 E-19$ & $3 E-16$ & $2 E-19$ & $4 E-19$ & $5 E-18$ & $5 E-11$ & $9 E-18$ & -18 \\
\hline 12 & $3.0738 \mathrm{E}-19$ & $6.2123 \mathrm{E}-16$ & $5 E-19$ & $3 E-19$ & $7 E-18$ & $2 E-13$ & $2 E-18$ & $8 \mathrm{E}-18$ \\
\hline 13 & $E-19$ & $E-16$ & $E-19$ & LE-19 & $2 E-18$ & -5 & E-18 & $E-19$ \\
\hline 14 & $2.5639 E-19$ & $4.0987 E-16$ & $2.2105 \mathrm{E}-19$ & $3.3037 \mathrm{E}-19$ & $7 E-18$ & $31 E-16$ & $46 \mathrm{E}-17$ & $3 \mathrm{E}-19$ \\
\hline 15 & $E-19$ & $E-16$ & $7 E-19$ & $E-19$ & BE-19 & $\mathrm{LE}-17$ & E-15 & -19 \\
\hline 16 & $E-19$ & $E-16$ & $\mathrm{E}-19$ & $5-19$ & $6 E-19$ & $2 E-18$ & $6 \mathrm{E}-14$ & $8 \mathrm{E}-19$ \\
\hline 17 & $2.0323 E-19$ & $2.1894 \mathrm{E}-16$ & $3 E-19$ & $2.5548 \mathrm{E}-19$ & $4 E-19$ & $8 E-18$ & $8 \mathrm{E}-12$ & อ2E-19 \\
\hline 18 & $E-19$ & $E-16$ & $E-19$ & $3 E-19$ & $E-19$ & $E-18$ & $E-10$ & $8 \mathrm{E}-19$ \\
\hline 19 & -19 & $E-16$ & $E-19$ & $E-19$ & $E-19$ & $\mathrm{E}-18$ & $E-09$ & $E-19$ \\
\hline 20 & $2.4927 \mathrm{E}-18$ & $E-15$ & $\mathrm{E}-18$ & $0 \mathrm{E}-18$ & $3 E-18$ & $2 E-17$ & $5 E-\odot 7$ & $1 E-18$ \\
\hline 21 & $2.3406 \mathrm{E}-18$ & $2.4070 \mathrm{E}-14$ & $2.0841 \mathrm{E}-18$ & $2.8417 \mathrm{E}-18$ & $4 E-18$ & $9 E-17$ & $6 \mathrm{E}-07$ & $76 E-18$ \\
\hline 22 & $9 \mathrm{E}-19$ & $3 E-15$ & $E-19$ & $\mathrm{E}-19$ & E-19 & $2 E-19$ & $1 E-\odot 9$ & $1 E-19$ \\
\hline 23 & $E-16$ & 3.04 & $E-16$ & $\mathrm{E}-15$ & E-15 & $\mathrm{E}-14$ & $5 E-06$ & $2 \mathrm{E}-15$ \\
\hline 24 & $2.5752 \mathrm{E}-17$ & $2.0325 \mathrm{E}-17$ & $2.2533 \mathrm{E}-17$ & $2.8564 \mathrm{E}-17$ & 41E-17 & $6 \mathrm{E}-17$ & $92 \mathrm{E}-10$ & $2.9549 \mathrm{E}-17$ \\
\hline 25 & $\mathrm{E}-12$ & $E-14$ & $\mathrm{E}-10$ & $\mathrm{E}-\odot 9$ & BE-17 & $5 \mathrm{E}-17$ & E-18 & $9 \mathrm{E}-12$ \\
\hline 26 & E- -7 & 1.2 & $5 E-10$ & E-๑8 & $8 E-16$ & $2 \mathrm{E}-17$ & $2 \mathrm{E}-18$ & $2.6373 \mathrm{E}-11$ \\
\hline otal & $2.4391 \mathrm{E}-06$ & $7.8338 E-06$ & $.6308 \mathrm{E}-06$ & $6.4591 \mathrm{E}-06$ & $1.7350 \mathrm{E}-\odot 7$ & 1. $3595 \mathrm{E}-07$ & $2.7468 \mathrm{E}-06$ & $7.9814 \mathrm{E}-06$ \\
\hline
\end{tabular}


APPENDIX B

PROJECT SCHEDULE 


\section{PROJECT SCHEDULE}

Sheet 1

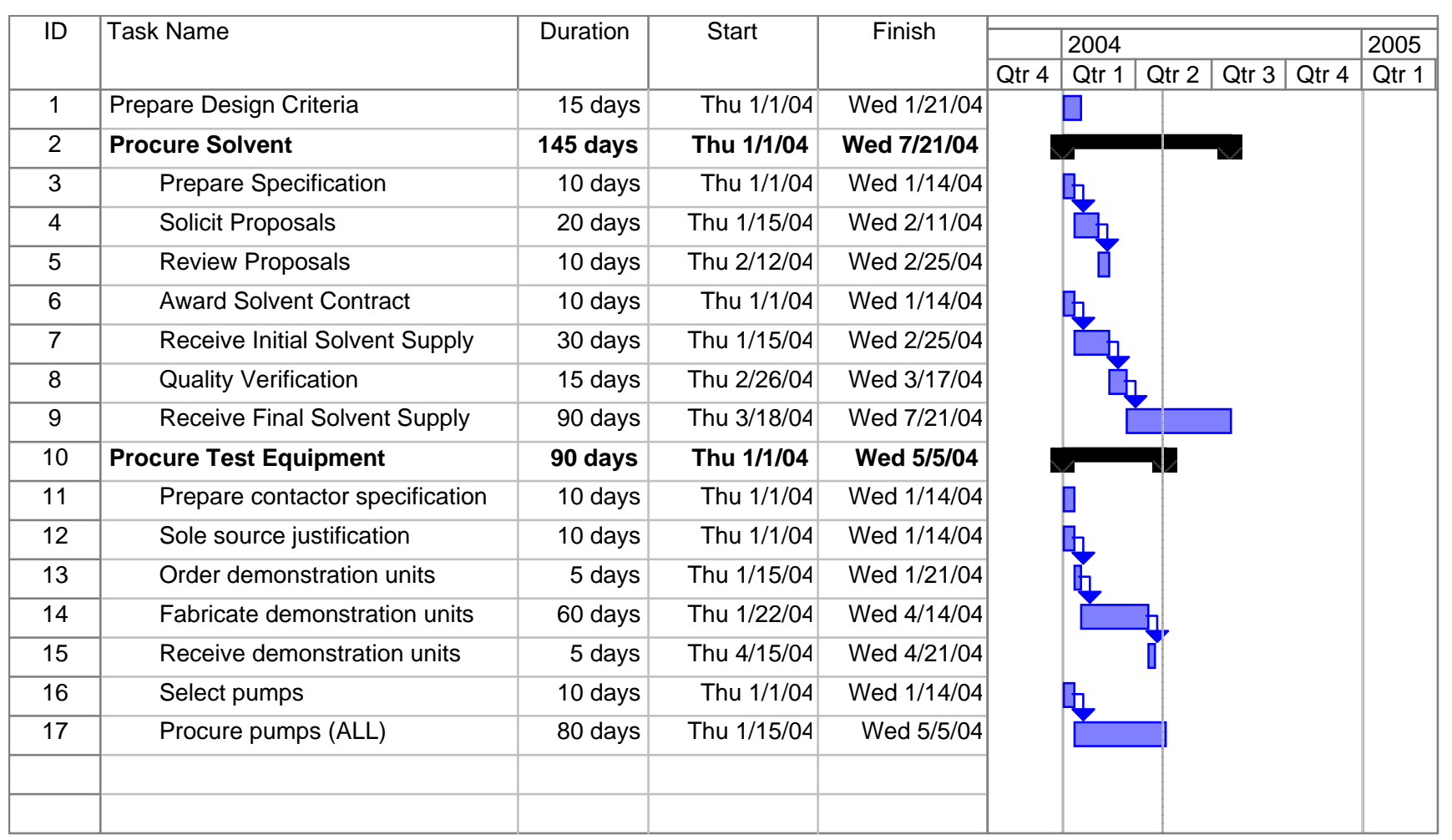


PROJECT SCHEDULE

Sheet 2

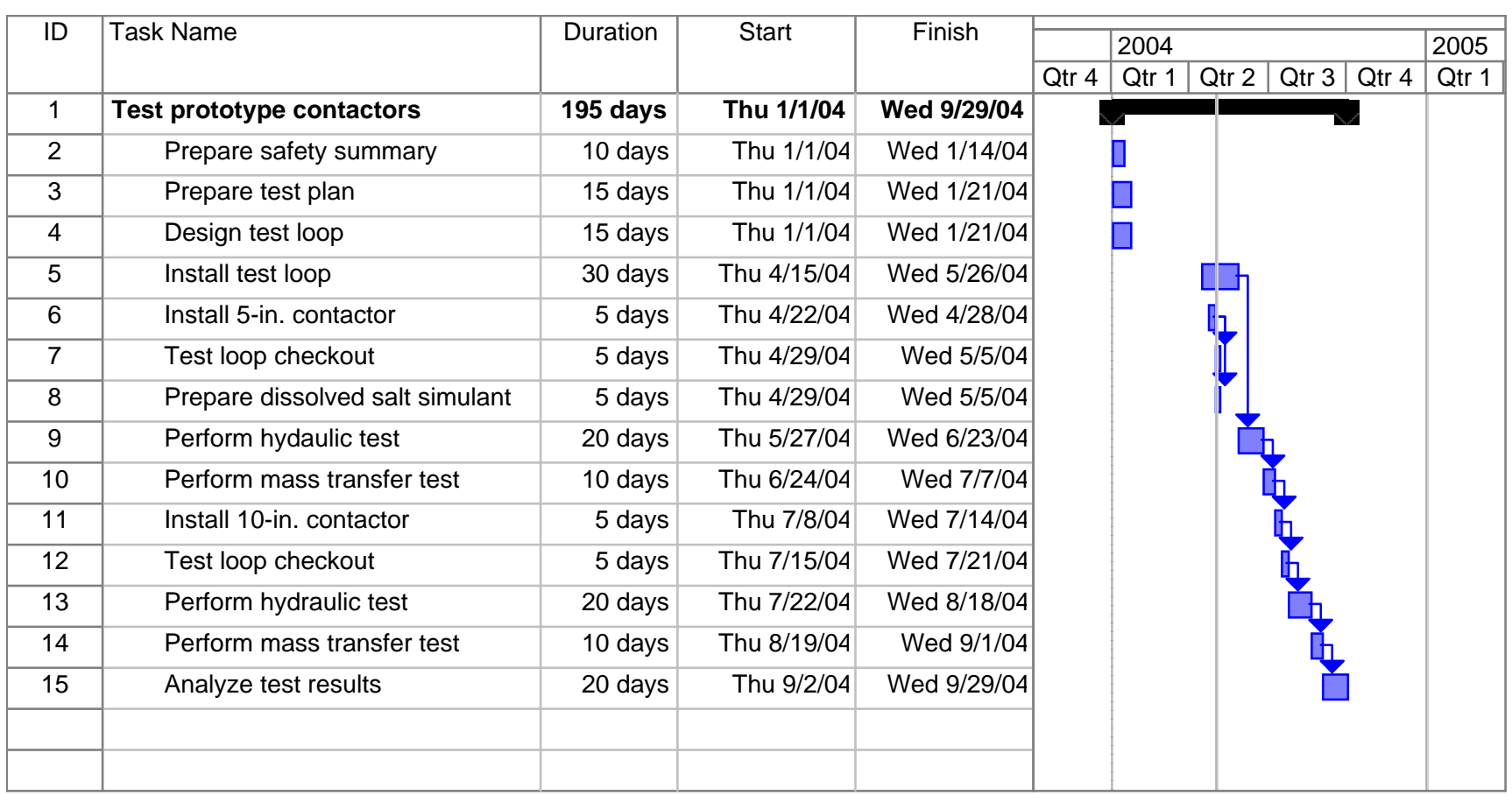




\section{PROJECT SCHEDULE}

Sheet 3

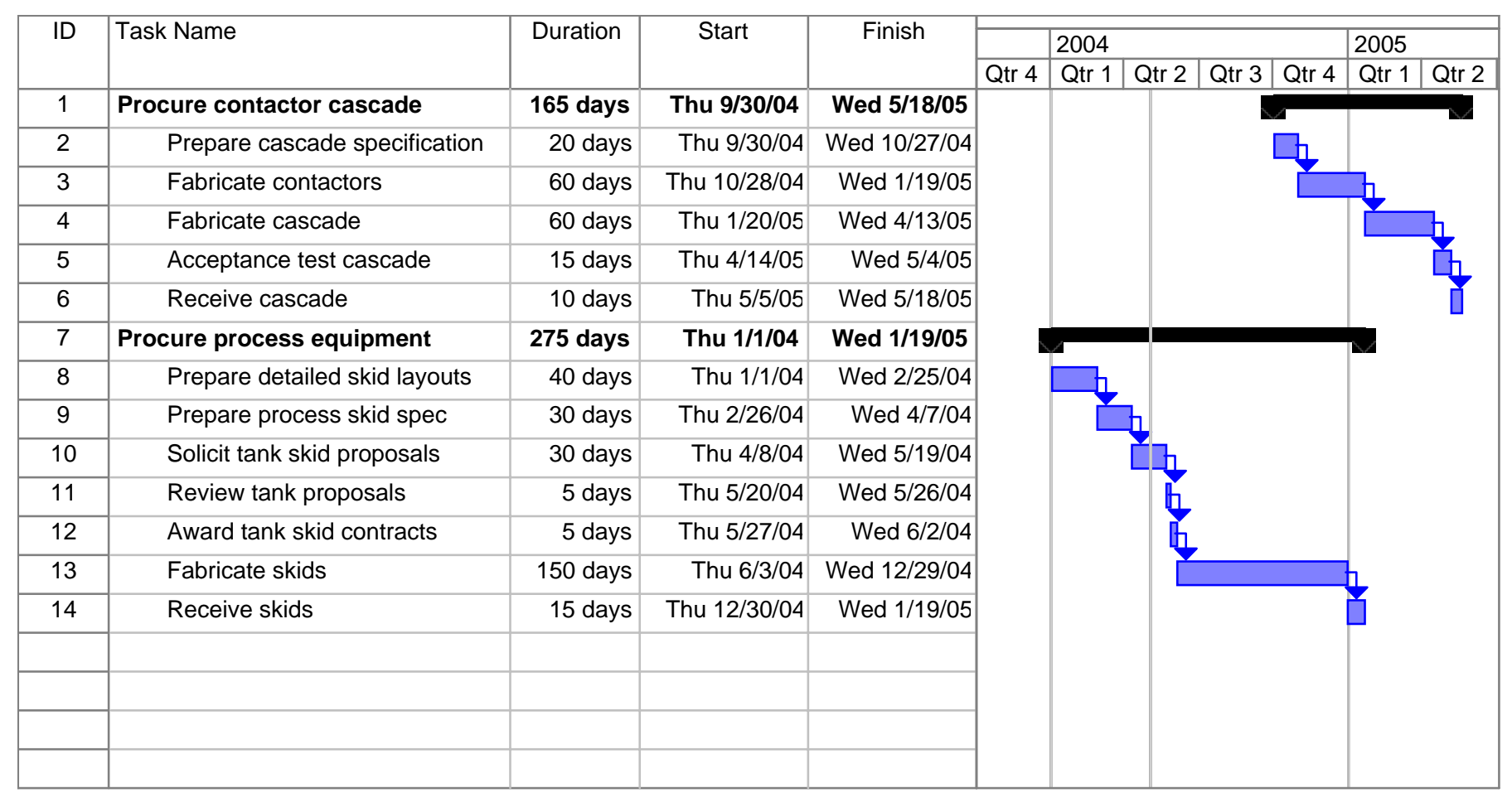




\section{PROJECT SCHEDULE}

Sheet 4

\begin{tabular}{|c|c|c|c|c|c|c|c|}
\hline \multirow[t]{2}{*}{ ID } & \multirow[t]{2}{*}{ Task Name } & \multirow[t]{2}{*}{ Duration } & \multirow[t]{2}{*}{ Start } & \multirow[t]{2}{*}{ Finish } & \multicolumn{2}{|r|}{2004} & \multirow{2}{*}{\begin{tabular}{|l|}
2005 \\
Qtr 1 \\
\end{tabular}} \\
\hline & & & & & Qtr 4 & \begin{tabular}{|l|l|l|l|} 
Qtr 1 & Qtr 2 & Qtr 3 & Qtr 4 \\
\end{tabular} & \\
\hline 2 & Perform final dose analysis & 20 days & Thu $2 / 26 / 04$ & Wed 3/24/04 & & & \\
\hline 3 & Design enclosure modifications & 40 days & Thu 3/25/04 & Wed 5/19/04 & & & \\
\hline 4 & Prepare enclosure specification & 20 days & Thu 5/20/04 & Wed 6/16/04 & & & \\
\hline 5 & Solicit enclosure proposals & 30 days & Thu $6 / 17 / 04$ & Wed $7 / 28 / 04$ & & & \\
\hline 6 & Fabricate enclosures & 100 days & Thu $7 / 29 / 04$ & Wed 12/15/04 & & & \\
\hline 7 & Receive enclosures & 20 days & Thu $12 / 16 / 04$ & Wed 1/12/05 & & & \\
\hline 8 & Prepare compliance documentation & 140 days & Thu $1 / 1 / 04$ & Wed 7/14/04 & & & \\
\hline 9 & Perform safety assessment & 80 days & Thu 1/1/04 & Wed 4/21/04 & & & \\
\hline 10 & Prepare environmental assessment & 80 days & Thu 1/1/04 & Wed 4/21/04 & & & \\
\hline 11 & Modify existing permits & 60 days & Thu $4 / 22 / 04$ & Wed $7 / 14 / 04$ & & & \\
\hline 12 & Prepare quality plan & 60 days & Thu 1/1/04 & Wed 3/24/04 & & & \\
\hline & & & & & & & \\
\hline
\end{tabular}




\section{PROJECT SCHEDULE}

Sheet 5

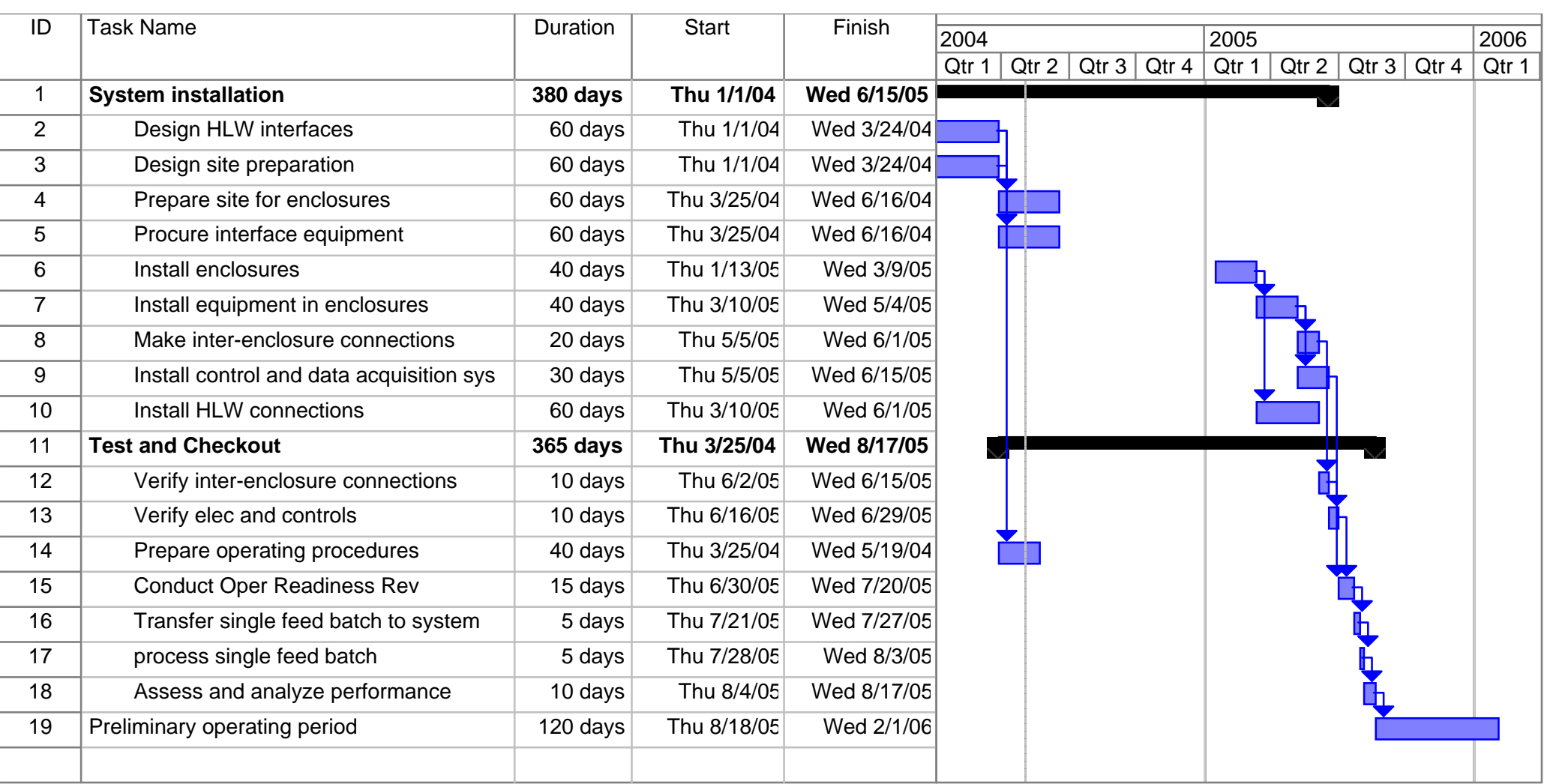





\section{ELECTRONIC DISTRIBUTION}

J. F. Birdwell, Oak Ridge National Laboratory

S. M. Blanco, Westinghouse Savannah River Company

P. V. Bonneson, Oak Ridge National Laboratory

S. G. Campbell, Westinghouse Savannah River Company

W. D. Clark, Westinghouse Savannah River Company

S. L. Clifford, Westinghouse Savannah River Company

D. T. Conrad, Westinghouse Savannah River Company

R. M. Counce, Oak Ridge National Laboratory

L. H. Delmau, Oak Ridge National Laboratory

H. H. Elder, Westinghouse Savannah River Company

S. D. Fink, Savannah River Technology Center

F. F. Fondeur, Savannah River Technology Center

B. A. Gifford, Westinghouse Savannah River Company

H. D. Harmon, Westinghouse Savannah River Company

D. T. Hobbs, Savannah River Technology Center

W. D. Kerley, Westinghouse Savannah River Company

D. P. Lambert, Savannah River Technology Center

C. A. Lanigan, Westinghouse Savannah River Company

R. K. Leugemours, Westinghouse Savannah River Company

B. E. Lewis, Oak Ridge National Laboratory

C. J. Martino, Westinghouse Savannah River Company

J. W. McCullough, Westinghouse Savannah River Company

L. T. McGuire, Westinghouse Savannah River Company

C. Miller, U. S. Department of Energy

B. A. Moyer, Oak Ridge National Laboratory

C. A. Nash, Savannah River Technology Center

M. A. Norato, Savannah River Technology Center

M. R. Norton, Westinghouse Savannah River Company

L. M. Papouchado, Savannah River Technology Center

T. M. Peters, Savannah River Technology Center

R. A. Pierce, Savannah River Technology Center

J. A. Pike, Savannah River Technology Center

S. H. Reboul, Savannah River Technology Center

S. J. Robertson, Savannah River Technology Center

B. C. Rogers, Westinghouse Savannah River Company

R. A. Runnels, Westinghouse Savannah River Company

J. E. Rushton, Oak Ridge National Laboratory

C. O. Slater, Oak Ridge National Laboratory

T. J. Spears, U. S. Department of Energy

R. H. Spires, Westinghouse Savannah River Company

M. E. Stallings, Savannah River Technology Center

W. E. Stevens, Savannah River Technology Center 
S. G. Subosits, Westinghouse Savannah River Company

P. C. Suggs, U. S. Department of Energy

G. A. Taylor, Savannah River Technology Center

P. A. Taylor, Oak Ridge National Laboratory

P. J. Valenti, Westinghouse Savannah River Company

W. B. Van-Pelt, Savannah River Technology Center

D. D. Walker, Savannah River Technology Center

J. F. Walker, Oak Ridge National Laboratory

A. O. Waring, Westinghouse Savannah River Company

F. A. Washburn, Westinghouse Savannah River Company

W. R. Wilmarth, Savannah River Technology Center

OTIC-RC, OSTI, CRL 